\title{
Thermodynamic metrics on outer space
}

\author{
TARIK AOUGAB $\dagger$, MATT CLAY \\ $\dagger$ Department of Mathematics, Haverford College, 370 Lancaster Avenue, \\ Haverford, PA 19041, USA \\ (e-mail:taougab@haverford.edu) \\ $\ddagger$ Department of Mathematics, University of Arkansas, Fayetteville, AR 72701, USA \\ (e-mail: mattclay@uark.edu,yoav@uark.edu)
}

(Received 27 January 2021 and accepted in revised form 29 November 2021)

\begin{abstract}
In this paper we consider two piecewise Riemannian metrics defined on the Culler-Vogtmann outer space which we call the entropy metric and the pressure metric. As a result of work of McMullen, these metrics can be seen as analogs of the Weil-Petersson metric on the Teichmüller space of a closed surface. We show that while the geometric analysis of these metrics is similar to that of the Weil-Petersson metric, from the point of view of geometric group theory, these metrics behave very differently than the Weil-Petersson metric. Specifically, we show that when the rank $r$ is at least 4, the action of $\operatorname{Out}\left(\mathbb{F}_{r}\right)$ on the completion of the Culler-Vogtmann outer space using the entropy metric has a fixed point. A similar statement also holds for the pressure metric.
\end{abstract}

Key words: outer space, automorphisms of free groups, thermodynamic formalism, Weill-Petersson metric

2020 Mathematics Subject Classification: 20F65 (Primary); 20E05, 57-XX (Secondary)

\section{Contents}

1 Introduction

1.1 Metrics on outer space

1.2 Thermodynamic metrics

1.3 Incompletion of the metric

1.4 The moduli space of the $r$-rose

1.5 A fixed point in the completion

1.6 Analogous statements for pressure metric

1.7 Further discussion and questions

2 Graphs and outer space 
3 Thermodynamic metrics 742

$\begin{array}{lll}3.1 & \text { Entropy } & 742\end{array}$

$\begin{array}{lll}3.2 & \text { Pressure } & 744\end{array}$

$\begin{array}{lll}3.3 & \text { Thermodynamic metrics } & 745\end{array}$

4 A determinant-defining equation for $\mathcal{M}^{1}(G) \quad 748$

$\begin{array}{lll}4.1 & \text { Determinant equation } & 748\end{array}$

$\begin{array}{ll}4.2 \text { A simplification } & 752\end{array}$

5 The topology induced by the entropy metric $\quad 754$

6 The entropy metric on $\mathcal{X}^{1}\left(\mathbb{F}_{2}\right) \quad 757$

$\begin{array}{lll}6.1 & \text { The 2-rose } & 757\end{array}$

$\begin{array}{ll}6.2 \text { The barbell graph } & 759\end{array}$

$\begin{array}{lll}6.3 & \text { The theta graph } & 760\end{array}$

$6.4\left(\mathcal{X}^{1}\left(\mathbb{F}_{2}\right), d_{\mathfrak{h}}\right)$ is complete $\quad 761$

7 The moduli space of the rose $\quad 762$

$\begin{array}{lll}7.1 & \mathcal{M}^{1}\left(\mathcal{R}_{r}\right) \text { as a zero locus } & 763\end{array}$

$\begin{array}{lll}7.2 & \text { Finite-length paths in } \mathcal{M}^{1}\left(\mathcal{R}_{r}\right) \text { for } r \geq 3 & 765\end{array}$

7.3 The diameter of $\mathcal{M}^{1}\left(\mathcal{R}_{r}\right)$ is infinite 768

8 Proof of Theorem $1.1 \quad 773$

9 The completion of $\left(\mathcal{M}^{1}\left(\mathcal{R}_{r}\right), d_{\mathfrak{h}, \mathcal{R}_{r}}\right) \quad 774$

$\begin{array}{lll}9.1 & \text { The model space } \widehat{M}^{1}\left(\mathcal{R}_{r}\right) & 774\end{array}$

$\begin{array}{lll}9.2 & \text { Proof of Theorem } 1.2 & 777\end{array}$

$\begin{array}{lll}9.3 & \text { The thin part of } \mathcal{M}^{1}\left(\mathcal{R}_{r}\right) & 778\end{array}$

10 The moduli space of a graph with a separating edge $\quad 781$

$\begin{array}{ll}10.1 \text { Finite-length paths in } \mathcal{M}^{1}(G) & 782\end{array}$

$\begin{array}{ll}10.2 \text { The model space } \widehat{\mathcal{M}}^{1}(G) & 785\end{array}$

$11 \mathcal{X}^{1}\left(\mathcal{R}_{r}\right.$, id) has bounded diameter in $\mathcal{X}^{1}\left(\mathbb{F}_{r}\right) \quad 788$

12 Proof of Theorem $1.3 \quad 790$

$\begin{array}{ll}\text { Acknowledgements } & 791\end{array}$

$\begin{array}{ll}\text { References } & 791\end{array}$

\section{Introduction}

The purpose of this paper is to introduce and examine two piecewise Riemannian metrics, called the entropy metric and the pressure metric, on the Culler-Vogtmann outer space $C V\left(\mathbb{F}_{r}\right)$. The Culler-Vogtmann outer space is the moduli space of unit-volume marked metric graphs and as such it is often viewed as the analog of the Teichmüller space of an orientable surface $S_{g}$. Both the Culler-Vogtmann outer space and the Teichmüller space admit a natural properly discontinuous action by a group. For the Culler-Vogtmann outer space, the group is the outer automorphism group of a free $\operatorname{group} \operatorname{Out}\left(\mathbb{F}_{r}\right)=$ $\operatorname{Aut}\left(\mathbb{F}_{r}\right) / \operatorname{Inn}\left(\mathbb{F}_{r}\right)$. For the Teichmüller space, the group is the mapping class group of the surface $\operatorname{MCG}\left(S_{g}\right)=\pi_{0}$ (Homeo $\left.^{+}\left(S_{g}\right)\right)$. Strengthening the connection between these spaces and groups are the facts that (i) $\operatorname{Out}\left(\mathbb{F}_{r}\right)$ is isomorphic to the group of homotopy equivalences of a graph whose fundamental group is isomorphic to $\mathbb{F}_{r}$, that is, $\operatorname{Out}\left(\mathbb{F}_{r}\right)$ can be thought of as the mapping class group of a graph, and (ii) the Dehn-Nielsen-Baer 
theorem which states that the extended mapping class group $\operatorname{MCG}^{ \pm}\left(S_{g}\right)$ (which also includes isotopy classes of orientation-reversing homeomorphisms) is isomorphic to $\operatorname{Out}\left(\pi_{1}\left(S_{g}\right)\right)$ [17]. This analogy has led to much fruitful research on the outer automorphism group of a free group $\operatorname{Out}\left(\mathbb{F}_{r}\right)$.

The metrics on the Culler-Vogtmann outer space we consider in this paper are analogs to the classical Weil-Petersson metric on the Teichmüller space of an orientable surface. The Weil-Petersson metric has been studied extensively from the point of view of both geometric analysis and geometric group theory. On the one hand, it enjoys many important analytic properties which can be expressed naturally in terms of hyperbolic geometry on $S_{g}$. Its utility in geometric group theory then stems from the fact that every isometry of the Weil-Petersson metric is induced by a mapping class [25]. Thus, the action of $\operatorname{MCG}\left(S_{g}\right)$ on the Teichmüller space equipped with the Weil-Petersson metric encodes information about useful invariants for mapping classes.

As the piecewise Riemannian metrics on the Culler-Vogtmann outer space that we study in this paper are motivated by the classical Weil-Petersson metric on the Teichmüller space of a closed surface, it is natural to ask to what extent they are true analogs of the Weil-Petersson metric. A major takeaway from the work in this paper is that they should be seen as natural analogs from the geometric analysis point of view, but not from the geometric group theory perspective. Specifically, while we highlight some similarities between these metrics and the Weil-Petersson metric as seen from the analytic point of view (Theorems 1.1 and 1.2) the main result (Theorem 1.3) of this paper shows that from the geometric group-theoretic perspective, these metrics are not useful (except possibly when $r=3$ ). The content of this theorem is summarized as follows: the action of $\operatorname{Out}\left(\mathbb{F}_{r}\right)$ on the metric completion of the Culler-Vogtmann outer space has a fixed point for $r \geq 4$. The remainder of this introduction discusses these metrics more thoroughly and provides context for the main results.

1.1. Metrics on outer space. The topology of $C V\left(\mathbb{F}_{r}\right)$ has been well studied; see, for instance, the survey papers of Bestvina [5] and Vogtmann [35]. The metric theory of $C V\left(\mathbb{F}_{r}\right)$ has been steadily developing over the past decade. What is desired is a theory that reflects the dynamical properties of the natural action by $\operatorname{Out}\left(\mathbb{F}_{r}\right)$, that further elucidates the connection between $\operatorname{Out}\left(\mathbb{F}_{r}\right)$ and $\operatorname{MCG}(S)$, and that leads to useful new discoveries.

The metric that has received the most attention to date is the Lipschitz metric. Points in the Culler-Vogtmann outer space are represented by triples $(G, \rho, \ell)$ where $G$ is a finite connected graph, $\rho: \mathcal{R}_{r} \rightarrow G$ is a homotopy equivalence where $\mathcal{R}_{r}$ is the $r$-rose, and $\ell$ is a function from the edges of $G$ to $(0, \infty)$ for which the sum of $\ell(e)$ over all edges of $G$ is equal to 1 . (See $\$ 2.2$ for complete details.) We think of the function $\ell$ as specifying the length of each edge and as such $\ell$ determines a metric on $G$ where the interior of each edge $e$ is locally isometric to the interval $(0, \ell(e))$. The Lipschitz distance between two unit-volume marked metric graphs $\left(G_{1}, \rho_{1}, \ell_{1}\right)$ and $\left(G_{2}, \rho_{2}, \ell_{2}\right)$ in $C V\left(\mathbb{F}_{r}\right)$ is defined by

$$
d_{\text {Lip }}\left(\left(G_{1}, \rho_{1}, \ell_{1}\right),\left(G_{2}, \rho_{2}, \ell_{2}\right)\right)=\log \inf \left\{\operatorname{Lip}(f) \mid f: G_{1} \rightarrow G_{2}, \rho_{2} \simeq f \circ \rho_{1}\right\},
$$

where $\operatorname{Lip}(f)$ is the Lipschitz constant of the function $f: G_{1} \rightarrow G_{2}$ using the metrics induced by $\ell_{1}$ and $\ell_{2}$. respectively. In general the function $d_{\text {Lip }}$ is not symmetric. As such, 
$d_{\text {Lip }}(\cdot, \cdot)$ is not a true metric, but an asymmetric metric. See $[1,2,20]$ for more on the asymmetric aspects of the Lipschitz metric.

Regardless, the Lipschitz metric has been essential in several recent developments for $\operatorname{Out}\left(\mathbb{F}_{r}\right)$. This is in part due to the fact that the Lipschitz metric connects the dynamical properties of an outer automorphism of $\mathbb{F}_{r}$ acting on $C V\left(\mathbb{F}_{r}\right)$ to its action on conjugacy classes-of elements and of free factors-in $\mathbb{F}_{r}$. Notable are the 'Bers-like proof' of the existence of train-tracks by Bestvina [6], the proof of hyperbolicity of the free factor complex by Bestvina and Feighn [8], and the proof of hyperbolicity of certain free group extensions by Dowdall and Taylor [18].

In this way, the Lipschitz metric is akin to the Teichmüller metric on Teichmüller space which was used to prove the corresponding statements for the mapping class group $[4,19$, 26]. One can also define the Lipschitz metric on Teichmüller space using the same idea as in (1.1), and in this setting it is oftentimes called Thurston's asymmetric metric [34]. This metric has seen renewed attention lately, in part due to the usefulness of the Lipschitz metric on $C V\left(\mathbb{F}_{r}\right)$.

As a result of McMullen's interpretation of the Weil-Petersson metric on Teichmüller space via tools from the thermodynamic formalism applied to the geodesic flow on the hyperbolic surface [27, Theorem 1.12], there exists a natural candidate for the Weil-Petersson metric on the Culler-Vogtmann outer space. This idea was originally pursued by Pollicott and Sharp [31].

1.2. Thermodynamic metrics. The metrics we consider in this paper arise from the tools of the thermodynamic formalism as developed by Bowen [9], Parry and Pollicott [29], Ruelle [32] and others. The central objects involved are the notions of entropy and pressure. For a graph $G$, these notions define functions

$$
\mathfrak{h}_{G}: \mathcal{M}(G) \rightarrow \mathbb{R} \quad \text { and } \quad \mathfrak{P}_{G}: \mathbb{R}^{n} \rightarrow \mathbb{R}
$$

where $n$ is the number of (geometric) edges in $G$ and $\mathcal{M}(G)=\mathbb{R}_{>0}^{n}$-this space parametrizes the length functions on $G$. The entropy and pressure functions are real analytic, strictly convex and are related by $\mathfrak{h}_{G}(\ell)=1$ if and only if $\mathfrak{P}_{G}(-\ell)=0$ (see Theorem 3.7). As these functions are smooth and strictly convex, their Hessians induce an inner product on the tangent space of the unit-entropy subspace $\mathcal{M}^{1}(G)=\{\ell \in \mathcal{M}(G) \mid$ $\left.\mathfrak{h}_{G}(\ell)=1\right\}$ at a length function (see Definition 3.10). Hence the notions of entropy and pressure induce Riemannian metrics on $\mathcal{M}^{1}(G)$ which we call the entropy metric and pressure metric, respectively. By $d_{\mathfrak{h}, G}$ and $d_{\mathfrak{P}, G}$ we denote the induced distance functions on $\mathcal{M}^{1}(G)$. We caution the reader that these metrics have been considered by others with conflicting terminology. Throughout this introduction, we will use the above terminology even when referencing the work of others. See Remark 3.11 for a further discussion.

Pollicott and Sharp initiated the study of the thermodynamic metrics when they first defined the pressure metric on $\mathcal{M}^{1}(G)$ [31]. They proved that the pressure metric is not complete for the 2-rose $\mathcal{R}_{2}$ and they derived formulas for the sectional curvature for the theta graph $\Theta_{2}$ and barbell graph $\mathcal{B}_{2}$ (see Figure 2 for these graphs). Additionally, Pollicott and Sharp produce a dynamical characterization of the entropy metric in terms of generic geodesics similar to Wolpert's result for the Weil-Petersson metric [37] (see Remark 
3.11). Kao furthered these results by showing that the pressure metric is incomplete for $\Theta_{2}, \mathcal{B}_{2}$ and the 3-rose $\mathcal{R}_{3}$, and by showing that the entropy metric is complete for $\mathcal{R}_{2}$ [22]. Additionally, he derives formulas for the sectional curvature with respect to both the entropy and the pressure metric for $\Theta_{2}, \mathcal{B}_{2}$ and $\mathcal{R}_{3}$. Xu shows that for certain graphs, the moduli space $\mathcal{M}^{1}(G)$ equipped with the entropy metric arises in the completion of the Teichmüller space of a surface with boundary using the pressure metric [39].

In this paper we will investigate the entropy metric not only on the moduli space of a single graph, but on the full moduli space of all marked graphs. Let $\mathcal{X}\left(\mathbb{F}_{r}\right)$ be the space of marked metric graphs so that contained in $\mathcal{X}\left(\mathbb{F}_{r}\right)$ is the Culler-Vogtmann outer space $C V\left(\mathbb{F}_{r}\right)$. The notion of entropy extends to $\mathcal{X}\left(\mathbb{F}_{r}\right)$ by $\mathfrak{h}([(G, \rho, \ell)])=\mathfrak{h}_{G}(\ell)$ and we set

$$
\mathcal{X}^{1}\left(\mathbb{F}_{r}\right)=\left\{[(G, \rho, \ell)] \in \mathcal{X}\left(\mathbb{F}_{r}\right) \mid \mathfrak{h}([(G, \rho, \ell)])=1\right\}
$$

There is a homeomorphism between $C V\left(\mathbb{F}_{r}\right)$ and $\mathcal{X}^{1}\left(\mathbb{F}_{r}\right)$ defined by scaling the length function (see §3.1). Fixing a graph $G$ and a marking $\rho: \mathcal{R}_{r} \rightarrow G$, the map $\mathcal{M}^{1}(G) \rightarrow$ $\mathcal{X}^{1}\left(\mathbb{F}_{r}\right)$ that sends a length function $\ell$ in $\mathcal{M}^{1}(G)$ to the point determined by $(G, \rho, \ell)$ in $\mathcal{X}^{1}\left(\mathbb{F}_{r}\right)$ is an embedding whose image we denote by $\mathcal{X}^{1}(G, \rho)$. Considering all marked graphs individually, this induces a piecewise Riemannian metric on $\mathcal{X}^{1}\left(\mathbb{F}_{r}\right)$. See $\$ 3.3$ for complete details. We denote the induced distance function on $\mathcal{X}^{1}\left(\mathbb{F}_{r}\right)$ by $d_{\mathfrak{h}}$.

For a closed orientable surface $S_{g}$, one can repeat the above discussion using the moduli space of marked Riemannian metrics with constant curvature $\mathcal{X}\left(S_{g}\right)$. In this case, the entropy and the area of the Riemann surface are directly related. In particular, the unit-entropy, constant-curvature metrics correspond to the hyperbolic metrics, that is, those with constant curvature equal to -1 , and hence to those with area equal to $2 \pi(2 g-2)$. In other words, the entropy and area normalizations on $\mathcal{X}\left(S_{g}\right)$ result in the same subspace, the Teichmüller space of the surface. McMullen proved that the ensuing entropy metric on the Teichmüller space is proportional to the Weil-Petersson metric [27, Theorem 1.12].

It is this connection between the entropy metric and the Weil-Petersson metric that drives the research in this paper. After introducing the framework for both the entropy and the pressure metrics in $\S 3$, we specialize the discussion to the entropy metric because of this connection to the Weil-Petersson metric. All of the main results of this paper have analogous statements for the pressure metric and the proofs are similar, and in most cases substantially easier. The statements for the pressure metric are given in §1.6.

It is not necessary for this paper, but we mention that building on work of McMullen, Bridgeman [10] and Bridgeman et al [11] used these same ideas to define a metric on the space of conjugacy classes of regular irreducible representations of a hyperbolic group into a special linear group.

In the next three subsections, we explain our main results on the entropy metric on $\mathcal{X}^{1}\left(\mathbb{F}_{r}\right)$ and their relation to the Weil-Petersson metric on Teichmüller space.

1.3. Incompletion of the metric. Our first main result concerns the completion of the entropy metric on $\mathcal{X}^{1}\left(\mathbb{F}_{r}\right)$. Wolpert showed that the Weil-Petersson metric on Teichmüller space is not complete [36]. Our first theorem shows that when $r \geq 3$, the same holds for the entropy metric on $\mathcal{X}^{1}\left(\mathbb{F}_{r}\right)$. 
THEOREM 1.1. The metric space $\left(\mathcal{X}^{1}\left(\mathbb{F}_{r}\right), d_{\mathfrak{h}}\right)$ is complete if $r=2$ and incomplete if $r \geq 3$.

For $r \geq 3$, this theorem is proved by exhibiting a finite-length path in $\mathcal{M}^{1}\left(\mathcal{R}_{r}\right)$ that exits every compact subset. This path is defined by sending the length of one edge in $\mathcal{R}_{r}$ to infinity, while shrinking the others to maintain unit entropy (Proposition 7.8). This strategy—also used by Pollicott and Sharp [31] and Kao [22]—-shows that $\left(\mathcal{M}^{1}\left(\mathcal{R}_{r}\right), d_{\mathfrak{h}, \mathcal{R}_{r}}\right)$ is incomplete. We further show in $\S 8$ that this path also exits every compact set in $\mathcal{X}^{1}\left(\mathbb{F}_{r}\right)$. As $d_{\mathfrak{h}, \mathcal{R}_{r}}$ is an upper bound to $d_{\mathfrak{h}}$ (when defined), this path still has finite length when considered in $\mathcal{X}^{1}\left(\mathbb{F}_{r}\right)$ and thus Theorem 1.1 follows.

The path described above illustrates a general method for producing paths that exit every compact subset and that have finite length in the entropy metric: deform the metric by sending the length of some collection of edges to infinity while shrinking the others to maintain unit entropy. So long as the complement of the collection supports a unit-entropy metric, this path will have finite length. This explains why $\left(\mathcal{X}^{1}\left(\mathbb{F}_{2}\right), d_{\mathfrak{h}}\right)$ is complete: any metric on a graph where every component has rank at most 1 has entropy equal to zero. In $\S 6$ we demonstrate the calculations required to prove that $\left(\mathcal{X}^{1}\left(\mathbb{F}_{2}\right), d_{\mathfrak{h}}\right)$ is complete.

This is completely analogous to the setting of the Weil-Petersson metric on Teichmüller space. In that setting, deforming a hyperbolic metric on $S_{g}$ by pinching a simple closed curve results in a path with finite length that exits every compact set. Moreover, the geometric analysis agrees. For the path in $\mathcal{M}^{1}\left(\mathcal{R}_{r}\right)$ described above, if we parametrize the long edge by $-\log (t)$ as $t \rightarrow 0$, then the entropy norm along this path is $O\left(t^{-1 / 2}\right)$, as shown in Proposition 7.8. For the path in the Teichmüller space of $S_{g}$, if we parametrize the curve which is being pinched by $t$ as $t \rightarrow 0$, then the Weil-Petersson norm along this path is $O\left(t^{-1 / 2}\right)[38, \S 7]$. Note that in this case, the length of the shortest curve that intersects the pinched one has length approximately $-\log (t)$.

1.4. The moduli space of the r-rose. Our second main result is concerned with the entropy metric on the moduli space of the $r$-rose $\mathcal{R}_{r}$.

THEOREM 1.2. The completion of $\left(\mathcal{M}^{1}\left(\mathcal{R}_{r}\right), d_{\mathfrak{h}, \mathcal{R}_{r}}\right)$ is homeomorphic to the complement of the vertices of an $(r-1)$-simplex.

The space $\mathcal{M}^{1}\left(\mathcal{R}_{r}\right)$ is homeomorphic to the interior of an $(r-1)$-simplex. The faces added in the completion for $d_{\mathfrak{h}, \mathcal{R}_{r}}$ correspond to unit-entropy metrics on subroses. Such a metric is obtained as the limit of a sequence of length functions on $\mathcal{R}_{r}$ by sending the length of a collection of edges to infinity and scaling the others to maintain unit entropy. Specifically, a $(k-1)$-dimensional face of the completion corresponds to the moduli space of unit-entropy metrics on a sub- $k$-rose. As before, intuitively, the vertices of the $(r-1)$-simplex are missing in the completion as there does not exist a unit-entropy metric on $\mathcal{R}_{1}$.

That unit-entropy metrics on subroses arise as points in the completion follows from the calculations provided for the proof of incompleteness in Theorem 1.1 and some continuity arguments. This is shown in $§ 9.1$. The difficult part of the proof of Theorem 1.2 is showing that any path in $\left(\mathcal{M}^{1}\left(\mathcal{R}_{r}\right), d_{\mathfrak{h}, \mathcal{R}_{r}}\right)$ that sends the length of one edge to 0 (and hence the 
lengths of the other edges to infinity) necessarily has infinite length. This argument appears in Lemma 7.10 and Proposition 7.14. In $\$ 9.2$ we combine these two facts to complete the proof of Theorem 1.2.

In Example 9.7 we compare the completion of $\left(\mathcal{M}^{1}\left(\mathcal{R}_{3}\right), d_{\mathfrak{h}, \mathcal{R}_{3}}\right)$ to the closure of the unit-volume metrics on $\mathcal{R}_{3}$ in the axes topology on $C V\left(\mathbb{F}_{3}\right)$ (see $\$ 2.2$ for definitions). By Theorem 1.2, the completion in the entropy metric is a 2-simplex without vertices, whereas the closure in the axes topology is a 2-simplex. More interestingly, the newly added edges and vertices are dual: edges in the entropy completion correspond to vertices in the axes closure and the missing vertices in the entropy completion correspond to the edges in the axes closure. This is explained in detail in Example 9.7 and illustrated in Figure 6.

While it is not necessary for Theorem 1.2, we mention that in $\$ 9.3$ we prove that the diameter of a cross-section of the $(r-1)$-simplex goes to 0 as the length of one of the edges goes to 0 , that is, as the cross-section moves out toward one of the missing vertices. In other words, the completion of $\left(\mathcal{M}^{1}\left(\mathcal{R}_{r}\right), d_{\mathfrak{h}, \mathcal{R}_{r}}\right)$ is geometrically akin to an ideal hyperbolic $(r-1)$-simplex; see Lemma 9.9.

1.5. A fixed point in the completion. Whereas the first two main results demonstrate the similarity between the geometric analysis for the Weil-Petersson metric on the Teichmüller space and the entropy metric on the Culler-Vogtmann outer space, our final main result provides a stark contrast between these two metrics with respect to geometric group theory.

THEOREM 1.3. The subspace $\left(\mathcal{X}^{1}\left(\mathcal{R}_{r}, i d\right) \cdot \operatorname{Out}\left(\mathbb{F}_{r}\right), d_{\mathfrak{h}}\right) \subset\left(\mathcal{X}^{1}\left(\mathbb{F}_{r}\right), d_{\mathfrak{h}}\right)$ is bounded if $r \geq 4$. Moreover, the action of $\operatorname{Out}\left(\mathbb{F}_{r}\right)$ on the completion of $\left(\mathcal{X}^{1}\left(\mathbb{F}_{r}\right), d_{\mathfrak{h}}\right)$ has a fixed point.

This subspace consists of the unit-entropy metrics on every marked $r$-rose. To illustrate the difference with respect to the setting of the Weil-Petersson metric on Teichmüller space, we mention the fact due to Daskalopoulos and Wentworth that pseudo-Anosov mapping classes have positive translation length in their action on the Teichmüller space [16]. In particular, the action of the mapping class group does not have a fixed point in the completion of Teichmüller space with the Weil-Petersson metric.

The first step in the proof of Theorem 1.3 is to show that the image of the inclusion map $\mathcal{M}^{1}\left(\mathcal{R}_{r}\right) \rightarrow \mathcal{X}^{1}\left(\mathcal{R}_{r}\right.$, id $) \subset \mathcal{X}^{1}\left(\mathbb{F}_{r}\right)$ has bounded diameter for $r \geq 4$. This result is particularly striking in contrast to Theorem 1.2, since implicit in that theorem is that the space $\left(\mathcal{M}^{1}\left(\mathcal{R}_{r}\right), d_{\mathfrak{h}, \mathcal{R}_{r}}\right)$ has infinite diameter. Boundedness of the image of $\mathcal{M}^{1}\left(\mathcal{R}_{r}\right)$ is achieved by showing that the map induced via Theorem 1.2, $\Phi: \Delta^{r-1}-V \rightarrow \widehat{x}^{1}\left(\mathbb{F}_{r}\right)$, extends to $\Delta^{r-1}$, where $\Delta^{r-1}$ is an $(r-1)$-simplex, $V \subset \Delta^{r-1}$ is the set of vertices, and $\hat{\mathcal{X}}^{1}\left(\mathbb{F}_{r}\right)$ is the completion of $\mathcal{X}^{1}\left(\mathbb{F}_{r}\right)$ for $d_{\mathfrak{h}}$. The existence of this extension shows that $\mathcal{X}^{1}\left(\mathcal{R}_{r}\right.$, id) lies in a compact set and hence is bounded.

In order to show that $\Phi: \Delta^{r-1}-V \rightarrow \widehat{x}^{1}\left(\mathbb{F}_{r}\right)$ extends to the set $V$, we show that $\Phi$ maps every $(k-1)$-dimensional face of $\Delta^{r-1}-V$ to a single point when $1<k<r-1$. This is shown in $\S 11$. This fact, together with the previously mentioned fact about the diameter of the cross-sections going to 0 , gives that $\Phi$ extends to the set $V$ and that the entire $(r-3)$-skeleton of $\Delta^{r-1}$ is mapped to a single point in $\widehat{x}^{1}\left(\mathbb{F}_{r}\right)$. The collapse of a 


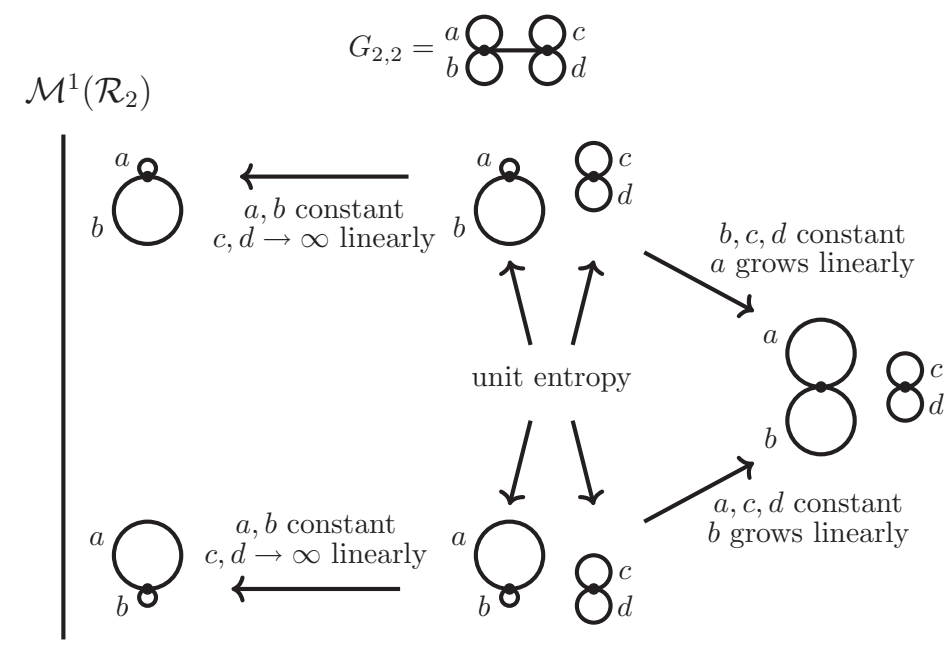

FIGURE 1. Illustration of a path with length 0 in the completion of $\left(\mathcal{M}^{1}\left(G_{2,2}\right), d_{\mathfrak{h}, G_{2,2}}\right)$.

$(k-1)$-dimensional face of $\Delta^{r-1}-V$ for $1<k<r-1$ arises from paths in $X^{1}\left(\mathbb{F}_{r}\right)$ connecting points in $\mathcal{X}^{1}\left(\mathcal{R}_{r}\right.$, id) whose length is much shorter than paths in $\mathcal{M}^{1}\left(\mathcal{R}_{r}\right)$ connecting the same points. In other words, there are shortcuts present in $\mathcal{X}^{1}\left(\mathbb{F}_{r}\right)$ that are not present in $\mathcal{M}^{1}\left(\mathcal{R}_{r}\right)$.

These shortcuts are most easily understood in terms of unit-entropy metrics on marked subgraphs, that is, points in the completion of $\left(\mathcal{X}^{1}\left(\mathbb{F}_{r}\right), d_{\mathfrak{h}}\right)$. Pathologies arise when the subgraph is not connected. In this case, the entropy of the metric on the subgraph is the maximum of the entropy-in the previous sense- on a component of the subgraph. Hence, by holding the length function constant on a component of the subgraph with unit entropy, we are free to modify the length function on the other components at will, so long as the entropy is never greater than 1 on any of these components. In Proposition 3.12 we show that the entropy and pressure metrics can be computed using the second derivatives of the lengths of edges along a path. Hence the length of a path that changes the length of the edges in a component with entropy less than 1 linearly has zero length in either of these metrics.

Figure 1 illustrates the central idea that is exploited in $\S 10$ to show that many paths have zero length. This figure is taking place in the completion of $\mathcal{M}^{1}\left(G_{2,2}\right)$ using the metric $d_{\mathfrak{h}, G_{2,2}}$. This space has $\mathcal{M}^{1}\left(\mathcal{R}_{4}\right)$ as a face in $\mathcal{X}^{1}\left(\mathbb{F}_{4}\right)$ corresponding to the collapse of the separating edge. The completion of $\mathcal{M}^{1}\left(\mathcal{R}_{4}\right)$ has an edge corresponding to unit-entropy metrics on two of the edges (denoted $a$ and $b$ in Figure 1). This edge also corresponds to a subset in the completion of $\mathcal{M}^{1}\left(G_{2,2}\right)$. Illustrated in Figure 1 is a path through unit-entropy length functions on subgraphs of $G_{2,2}$, that is, points in the completion. As all edge lengths are changing linearly, this path has length 0 and hence all of these length functions correspond to the same point in the completion.

This shows that the edge corresponding to $\mathcal{M}^{1}\left(\mathcal{R}_{2}\right)$ is mapped by $\Phi$ to a point. The same idea works for any sub- $k$-rose of $\mathcal{R}_{r}$ so long as $1<k<r-1$ : it is necessary to separate 
two subroses, each of which supports a unit-entropy metric. This is the reason why we require $r \geq 4$ in Theorem 1.3.

Once we know that the entire $(r-3)$-skeleton of $\Delta^{r-1}$ is mapped by $\Phi$ to a point in $\widehat{x}^{1}\left(\mathbb{F}_{r}\right)$, we utilize the structure of the Culler-Vogtmann outer space to conclude in $\$ 12$ that this point is independent of the marking $\rho: \mathcal{R}_{r} \rightarrow \mathcal{R}_{r}$ used to define the inclusion $\mathcal{M}^{1}\left(\mathcal{R}_{r}\right) \rightarrow \mathcal{X}^{1}\left(\mathbb{F}_{r}\right)$. This completes the proof of Theorem 1.3.

1.6. Analogous statements for pressure metric. For the pressure metric on $\mathcal{X}^{1}\left(\mathbb{F}_{r}\right)$ we have the following analogs of Theorems 1.1-1.3. By $d_{\mathfrak{P}}$ we denote the induced distance function.

(1) The space $\left(\mathcal{X}^{1}\left(\mathbb{F}_{r}\right), d_{\mathfrak{P}}\right)$ is incomplete for $r \geq 2$.

(2) The completion of $\left(\mathcal{M}^{1}\left(\mathcal{R}_{r}\right), d_{\mathfrak{P}, \mathcal{R}_{r}}\right)$ is homeomorphic to an $(r-1)$-simplex.

(3) The space $\left(\mathcal{X}^{1}\left(\mathbb{F}_{r}\right), d_{\mathfrak{P}}\right)$ is bounded if $r \geq 2$; moreover, the action of $\operatorname{Out}\left(\mathbb{F}_{r}\right)$ on the completion of $\left(\mathcal{X}^{1}\left(\mathbb{F}_{r}\right), d_{\mathfrak{P}}\right)$ has a fixed point.

These can be shown using techniques similar-and simpler- to those in this paper. The key source of the distinction between the entropy and pressure metrics is that the length function that assigns 0 to the unique edge on $\mathcal{R}_{1}$ has pressure equal to 0 even through the entropy is not defined. Hence the path in $\mathcal{M}^{1}\left(\mathcal{R}_{2}\right)$ that sends the length of one edge to infinity while shrinking the length of the other (necessarily to 0 ) to maintain unit entropy has finite length in the pressure metric, whereas the length in the entropy metric is infinite.

\subsection{Further discussion and questions. This work raises a number of questions.}

Our proof that the action of $\operatorname{Out}\left(\mathbb{F}_{r}\right)$ on the completion of $\left(\mathcal{X}^{1}\left(\mathbb{F}_{r}\right), d_{\mathfrak{h}}\right)$ has a fixed point relies heavily on the assumption that $r \geq 4$ : the key construction uses an edge that separates a given graph into two subgraphs, each with rank at least 2 . This leaves the door open to a negative answer for the following question, which would allow for interesting applications specifically for $\mathbb{F}_{3}$.

Question 1.4. Does $\left(\mathcal{X}^{1}\left(\mathbb{F}_{3}\right), d_{\mathfrak{h}}\right)$ admit an $\operatorname{Out}\left(\mathbb{F}_{3}\right)$-invariant bounded subcomplex?

Theorem 1.3 demonstrates the existence of an $\operatorname{Out}\left(\mathbb{F}_{r}\right)$ orbit in $\left(\mathcal{X}^{1}\left(\mathbb{F}_{r}\right), d_{\mathfrak{h}}\right)$ with bounded diameter but we do not yet know that the entire space has bounded diameter. We therefore ask the following question.

Question 1.5. Is $\left(\mathcal{X}^{1}\left(\mathbb{F}_{r}\right), d_{\mathfrak{h}}\right)$ bounded for $r \geq 4$ ?

We believe the answer to this question is yes. Indeed, the only way $\left(\mathcal{X}^{1}\left(\mathbb{F}_{r}\right), d_{\mathfrak{h}}\right)$ could fail to be bounded is if the subspace $\left(\mathcal{X}^{1}(G, \rho), d_{\mathfrak{h}}\right)$ has infinite diameter for some marked graph $\rho: \mathcal{R}_{r} \rightarrow G$. As the diameter of $\left(\mathcal{X}^{1}\left(\mathcal{R}_{r}, \mathrm{id}\right) \cdot \operatorname{Out}\left(\mathbb{F}_{r}\right), d_{\mathfrak{h}}\right)$ is bounded, to answer the question in the affirmative, it would suffice to find a bound (in terms of $r$ ) on the distance from any point $\mathcal{X}^{1}\left(\mathbb{F}_{r}\right)$ to a point in $\mathcal{X}^{1}\left(\mathcal{R}_{r}\right.$, id $) \cdot \phi$ for some $\phi \in \operatorname{Out}\left(\mathbb{F}_{r}\right)$. Another approach to answer Question 1.5 in the affirmative would be to show the existence of a bound (in terms of $r$ ) on distance from any point in $X^{1}(G, \rho)$ to a completion point represented by a unit-entropy metric on a proper subgraph (with the goal of getting to a point in the completion of a marked rose via induction). This led us to the following question, which is of independent interest and we pose here as a conjecture. 
Conjecture 1.6. For any $r \geq 3$ there exists $C>0$ so that any metric graph of rank $r$ with unit entropy contains a proper subgraph with entropy at least $C$.

It suffices to show the conjecture for a fixed topological type of graph since, for a given rank $r$, there are only finitely many topological types of graph of rank $r$.

One can also define the notion of the entropy metric on the Teichmüller space of a surface with boundary. In [39], Xu shows that this metric is incomplete. As mentioned previously, McMullen proved that for closed surfaces, the entropy metric is a constant multiple of the Weil-Petersson metric. However, by partially characterizing the completion of the entropy metric in the bordered setting, $\mathrm{Xu}$ is able to show that this is not true in the presence of boundary. Concretely, Xu identifies certain graphs $G$ so that, in the notation of this paper, $\left(\mathcal{M}^{1}(G), d_{\mathfrak{h}}\right)$ isometrically embeds in the completion of the Teichmüller space of the surface equipped with the entropy metric. We therefore ask if the work in this paper can be used to fully understand the completion of the Teichmüller space of a bordered surface equipped with the entropy metric.

Problem 1.7. Fully characterize the completion of $\left(\mathcal{M}^{1}(G), d_{\mathfrak{h}, G}\right)$ for an arbitrary graph $G$ and use this to study the completion of the Teichmüller space of a bordered surface, equipped with the entropy metric.

The pathology exhibited by Theorem 1.3 relies on the existence of a sequence of unit-entropy length functions whose limiting metric is supported on a subgraph with multiple components where the metric on some component need not have entropy equal to 1 . This behavior does not occur in the Teichmüller space of a closed surface since a unit-entropy metric on a constant-curvature surface is a hyperbolic metric and vice versa, and thus for the subsurface supporting the limit of a sequence of unit-entropy metrics, the metric on each component also has entropy equal to 1. One can also consider an entropy function defined over the moduli space of singular flat metrics on a closed surface. This setting appears more similar to the situation of Theorem 1.3 in that the unit-entropy condition is not encoded by the local geometry. It appears likely that some version of Theorem 1.3 holds for singular flat metrics, and so we therefore ask our final question of this introduction.

Question 1.8. Can the techniques used in this paper in the setting of metric graphs apply to the study of an entropy metric on the moduli space of singular flat metrics on a closed surface?

\section{Graphs and outer space}

In this section we introduce some concepts that are necessary for the sequel. First, we set some notation for dealing with graphs. Then we define the Culler-Vogtmann outer space—including its topology—and the $\operatorname{Out}\left(\mathbb{F}_{r}\right)$ action on this space.

2.1. Graphs. We use Serre's convention for graphs [33]. That is, an (undirected) graph is a tuple $G=\left(V, E, o, \tau,{ }^{-}\right)$where:

(1) $V$ and $E$ are sets, called the vertices and the directed edges (we think of $E$ as containing two copies, with opposite orientations, of each undirected edge); 
(2) $o, \tau: E \rightarrow V$ are functions that specify the originating and terminating vertices of an edge;

(3) ${ }^{-}: E \rightarrow E$ is a fixed point free involution such that $o(e)=\tau(\bar{e})$ ( ${ }^{-}$flips edges).

We fix an orientation on $G$, that is, a subset $E_{+} \subset E$ that contains exactly one edge from each pair $\{e, \bar{e}\}$. Since we consider the pair $\{e, \bar{e}\}$ to be a single edge, the number of edges of $G$ is $\left|E_{+}\right|=|E| / 2$. The valance of a vertex $v$ is the number of edges from $e \in E_{+}$ with $o(e)=v$ plus the number of edges from $e \in E_{+}$with $\tau(e)=v$ (an edge $e$ for which $o(e)=\tau(e)=v$ contributes 2 to the valance). Oftentimes when defining a graph we only specify the edges in $E_{+}$(together with the restrictions of $o$ and $\tau$ to $E_{+}$). The complete set of edges is then defined as $E=E_{+} \cup \bar{E}_{+}$, where $\bar{E}_{+}$is a copy of $E_{+}$, and $o, \tau$, and ${ }^{-}$are defined in the obvious way. We blur the distinction between the tuple $\left(V, E, o, \tau,{ }^{-}\right)$and the corresponding one-dimensional $\mathrm{CW}$-complex with 0-cells $V$ and 1-cells $E_{+}$.

The space of length functions on $G$ is the open convex cone

$$
\mathcal{M}(G)=\left\{\ell: E_{+} \rightarrow \mathbb{R}_{>0}\right\}
$$

We consider this set as a subset of $\mathbb{R}^{\left|E_{+}\right|}$. A length function $\ell: E_{+} \rightarrow \mathbb{R}_{>0}$ extends to a function $\ell: E \rightarrow \mathbb{R}_{>0}$ by $\ell(e)=\ell(\bar{e})$ if $e \notin E_{+}$. By $\mathbf{1} \in \mathcal{M}(G)$ we denote the constant function with value 1 .

An edge path is a sequence of edges $\left(e_{1}, \ldots, e_{n}\right)$ in $E$ such that $\tau\left(e_{i}\right)=o\left(e_{i+1}\right)$ for $i=1, \ldots, n-1$. A function $f: E \rightarrow \mathbb{R}$ (in particular, a length function) extends to a function on edge paths $\gamma=\left(e_{1}, \ldots, e_{n}\right)$ by

$$
f(\gamma)=\sum_{i=1}^{n} f\left(e_{i}\right) .
$$

2.2. Outer space. We will introduce some definitions and notation for the CullerVogtmann outer space. This space was originally defined by Culler and Vogtmann [14]. For more information, see for example the survey papers by Vogtmann [35] or Bestvina [5].

Let $\mathcal{R}_{r}$ be the $r$-rose. That is, $\mathcal{R}_{r}$ the graph with a unique vertex $v$ and $r$ edges. Fix an isomorphism $\mathbb{F}_{r} \cong \pi_{1}\left(\mathcal{R}_{r}, v\right)$. A marked metric graph (of rank $r$ ) is a triple $(G, \rho, \ell)$ where:

(1) $G$ is a finite connected graph without vertices of valence 1 or 2;

(2) $\rho: \mathcal{R}_{r} \rightarrow G$ is a homotopy equivalence; and

(3) $\ell$ is a length function on $G$.

There is an equivalence relation on the set of marked metric graphs defined by $\left(G_{1}, \rho_{1}, \ell_{1}\right) \sim\left(G_{2}, \rho_{2}, \ell_{2}\right)$ if there exists a graph automorphism $\alpha: G_{1} \rightarrow G_{2}$ such that $\ell_{1}=\ell_{2} \circ \alpha$ and such that the following diagram commutes up to homotopy:

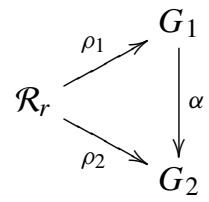


We let $\mathcal{X}\left(\mathbb{F}_{r}\right)$ denote the set of equivalence classes of marked metric graphs of rank $r$. The group $\operatorname{Out}\left(\mathbb{F}_{r}\right)$ acts on $\mathcal{X}\left(\mathbb{F}_{r}\right)$ on the right by precomposing the marking. Specifically, for any outer automorphism $\phi \in \operatorname{Out}\left(\mathbb{F}_{r}\right)$, there is a homotopy equivalence $g_{\phi}: \mathcal{R}_{r} \rightarrow \mathcal{R}_{r}$ that induces $\phi$ on $\pi_{1}\left(\mathcal{R}_{r}\right)$ via the aforementioned fixed isomorphism $\mathbb{F}_{r} \cong$ $\pi_{1}\left(\mathcal{R}_{r}, *\right)$. Moreover, this homotopy equivalence is unique up to homotopy. With this, we define

$$
(G, \rho, \ell) \cdot \phi=\left(G, \rho \circ g_{\phi}, \ell\right)
$$

This action respects the equivalence relation on marked metric graphs and so defines an action on $\mathcal{X}\left(\mathbb{F}_{r}\right)$ as claimed.

Let $\mathcal{G}_{r}$ denote the set of finite connected graphs without vertices of valence 1 or 2 whose fundamental group has rank $r$. We observe that this is a finite set. Given a graph $G \in \mathcal{G}_{r}$ and homotopy equivalence $\rho: \mathcal{R}_{r} \rightarrow G$, we set

$$
\mathcal{X}(G, \rho)=\left\{\left[\left(G_{0}, \rho_{0}, \ell_{0}\right)\right] \in \mathcal{X}\left(\mathbb{F}_{r}\right) \mid G_{0}=G \text { and } \rho_{0} \simeq \rho\right\} .
$$

There is a bijection $\mathcal{X}(G, \rho) \rightarrow \mathcal{M}(G)$ defined by $\left[\left(G_{0}, \rho_{0}, \ell_{0}\right)\right] \mapsto \ell_{0}$. These sets partition the set $\mathcal{X}\left(\mathbb{F}_{r}\right)$ and are permuted under the action by $\operatorname{Out}\left(\mathbb{F}_{r}\right)$. Specifically, for each $G \in \mathcal{G}_{r}$ we fix a marking $\rho_{G}: \mathcal{R}_{r} \rightarrow G$. Then

$$
\mathcal{X}\left(\mathbb{F}_{r}\right)=\bigcup_{G \in \mathcal{G}_{r}} \bigcup_{\phi \in \operatorname{Out}\left(\mathbb{F}_{r}\right)} \mathcal{X}\left(G, \rho_{G}\right) \cdot \phi .
$$

There is a topology on $\mathcal{X}\left(\mathbb{F}_{r}\right)$ that is often defined in three different ways. We will need to use the first two and for completeness we explain all three here.

The weak topology. The notion of a collapse induces a partial order on the set of marked graphs. Specifically, for two graphs $G$ and $G_{0}$, we say that $G$ collapses to $G_{0}$ if there is a surjection $c: G \rightarrow G_{0}$ such that the image of any edge in $G$ is either a vertex or an edge of $G_{0}$ and such that $c^{-1}(x)$ is a contractible subgraph of $G$ for each point $x$ of $G_{0}$. The map $c$ is a called a collapse. Observe that if the map $c: G \rightarrow G_{0}$ is a collapse, then a length function $\ell \in \mathcal{M}\left(G_{0}\right)$ can be considered as a degenerate length function $\ell_{G_{0}}$ on $G$ by

$$
\ell_{G_{0}}(e)= \begin{cases}\ell(c(e)) & \text { if } c(e) \text { is an edge in } G_{0} \\ 0 & \text { otherwise }\end{cases}
$$

This defines a map $c^{*}: \mathcal{M}\left(G_{0}\right) \rightarrow \mathbb{R}_{\geq 0}^{\left|E_{+}\right|}$by $c^{*}(\ell)=\ell_{G_{0}}$. We now define the following subset of $\mathbb{R}_{\geq 0}^{\left|E_{+}\right|}$:

$$
\overline{\mathcal{M}}(G)=\bigcup_{c: G \rightarrow G_{0}} c^{*}\left(\mathcal{M}\left(G_{0}\right)\right) .
$$

We note the $\mathcal{M}(G)$ is a subset of $\overline{\mathcal{M}}(G)$ as the identity map id: $G \rightarrow G$ is a collapse.

Next, given two marked graphs $\rho: \mathcal{R}_{r} \rightarrow G$ and $\rho_{0}: \mathcal{R}_{r} \rightarrow G_{0}$, we say that $(G, \rho)$ collapses to $\left(G_{0}, \rho_{0}\right)$ if there is a collapse $c: G \rightarrow G_{0}$ such that $\rho_{0} \simeq c \circ \rho$. In this case 
we write $\left(G_{0}, \rho_{0}\right) \leq(G, \rho)$. We now define the following subset of $\mathcal{X}\left(\mathbb{F}_{r}\right)$ :

$$
\bar{X}(G, \rho)=\bigcup_{\left(G_{0}, \rho_{0}\right) \leq(G, \rho)} \mathcal{X}\left(G_{0}, \rho_{0}\right) .
$$

The bijection $\mathcal{X}(G, \rho) \rightarrow \mathcal{M}(G)$ extends in a natural way to a bijection $\overline{\mathcal{X}}(G, \rho) \rightarrow \overline{\mathcal{M}}(G)$ and allows us to consider $\bar{X}(G, \rho)$ as a subset of $\mathbb{R}_{>0}^{\left|E_{+}\right|}$.

The weak topology is defined using this collection of subsets. Specifically, a set $U \subseteq$ $\mathcal{X}\left(\mathbb{F}_{r}\right)$ is open if $U \cap \overline{\mathcal{X}}(G, \rho)$ is open as a subset of $\mathbb{R}_{\geq 0}^{\left|E_{+}\right|}$for all marked graphs $(G, \rho)$.

The axes topology. Given a marked metric graph $(G, \rho, \ell)$ and an element $g \in \mathbb{F}_{r}$, we denoted by $\ell([g])$ the $\ell$-length of the shortest loop in $G$ representing the conjugacy class $[\rho(g)]$. This induces a function Len: $\mathcal{X}\left(\mathbb{F}_{r}\right) \rightarrow \mathbb{R}_{\geq 0}^{\mathbb{F}_{r}}$ where Len $([(G, \rho, \ell)]): \mathbb{F}_{r} \rightarrow \mathbb{R}_{\geq 0}$ is the function defined by

$$
\operatorname{Len}([(G, \rho, \ell)])(g)=\ell([g]) .
$$

Culler and Morgan proved that the map Len is injective [13, 3.7 Theorem]. The resulting subspace topology on $\operatorname{Len}\left(\mathcal{X}\left(\mathbb{F}_{r}\right)\right) \subset \mathbb{R}_{\geq 0}^{\mathbb{F}_{r}}$ is called the axes topology. It is known that this topology agrees with the weak topology. (See $[14, \S 1.1]$ or [21, Proposition 5.4].)

The equivariant Gromov-Hausdorff topology. We will not need this definition, and we only remark that Paulin showed that it is equivalent to the axes topology [30, Main Theorem].

There is an action of $\mathbb{R}_{>0}$ on $\mathcal{X}\left(\mathbb{F}_{r}\right)$ given by scaling the length function. Specifically, $a \cdot(G, \rho, \ell)=(G, \rho, a \cdot \ell)$. The quotient of $\mathcal{X}\left(\mathbb{F}_{r}\right)$ is denoted $\mathbb{P} \mathcal{X}\left(\mathbb{F}_{r}\right)$.

There are many continuous sections of the quotient map $\mathcal{X}\left(\mathbb{F}_{r}\right) \rightarrow \mathbb{P} \mathcal{X}\left(\mathbb{F}_{r}\right)$. An often used choice uses the notion of the volume of a length function, $\operatorname{vol}(\ell)$, that we define now. For a length function $\ell \in \mathcal{M}(G)$, we define the volume of $\ell$ by $\operatorname{vol}(\ell)=\sum_{e \in E_{+}} \ell(e)$. There is a section $\mathcal{V}: \mathbb{P} \mathcal{X}\left(\mathbb{F}_{r}\right) \rightarrow \mathcal{X}\left(\mathbb{F}_{r}\right)$ defined by

$$
\mathcal{V}([[(G, \rho, \ell)]])=\left[\left(G, \rho, \frac{1}{\operatorname{vol}(\ell)} \ell\right)\right] .
$$

We denote the image of this section by $C V\left(\mathbb{F}_{r}\right)$; it is known as the Culler-Vogtmann outer space. Further, given a marked graph $\rho: \mathcal{R}_{r} \rightarrow G$, we set $C V(G, \rho)=\mathcal{X}(G, \rho) \cap$ $C V\left(\mathbb{F}_{r}\right)$. This set is homeomorphic to an open simplex of dimension $\left|E_{+}\right|-1$.

Example 2.1. There are three graphs in $\mathcal{G}_{2}$ : the 2-rose $\mathcal{R}_{2}$, the theta graph $\Theta_{2}$ and the barbell graph $\mathcal{B}_{2}$; see Figure 2. Figure 3 shows a portion of $C V\left(\mathbb{F}_{2}\right)$ and how these simplices piece together. The homotopy equivalences used for Figure 3 are as follows:

$$
\begin{aligned}
\rho_{\Theta}: \mathcal{R}_{2} & \rightarrow \Theta_{2}: e_{1} \mapsto e_{1} \bar{e}_{3}, e_{2} \mapsto e_{2} \bar{e}_{3} ; \\
\rho_{\mathcal{B}}: \mathcal{R}_{2} & \rightarrow \mathcal{B}_{2}: e_{1} \mapsto e_{1}, e_{2} \mapsto e_{3} e_{2} \bar{e}_{3} ; \\
a: & \mathcal{R}_{2} \rightarrow \mathcal{R}_{2}: e_{1} \mapsto e_{1}, e_{2} \mapsto \bar{e}_{2} ; \\
b: & \mathcal{R}_{2} \rightarrow \mathcal{R}_{2}: e_{1} \mapsto \bar{e}_{2}, e_{2} \mapsto e_{1} \bar{e}_{2} .
\end{aligned}
$$

Notice that $\rho_{\Theta}$ is the homotopy inverse to the map $\Theta_{2} \rightarrow \mathcal{R}_{2}$ that collapses the edge $e_{3}$. Likewise $\rho_{\Theta} \circ b$ is the homotopy inverse to the collapse of $e_{2}$ and $\rho_{\Theta} \circ b^{2}$ is the homotopy 

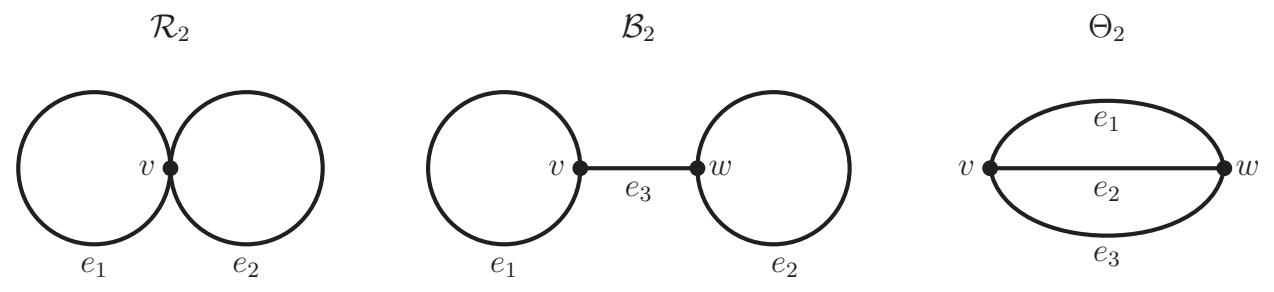

FIGURE 2. The three homeomorphism types of graphs in $\mathcal{G}_{2}$.

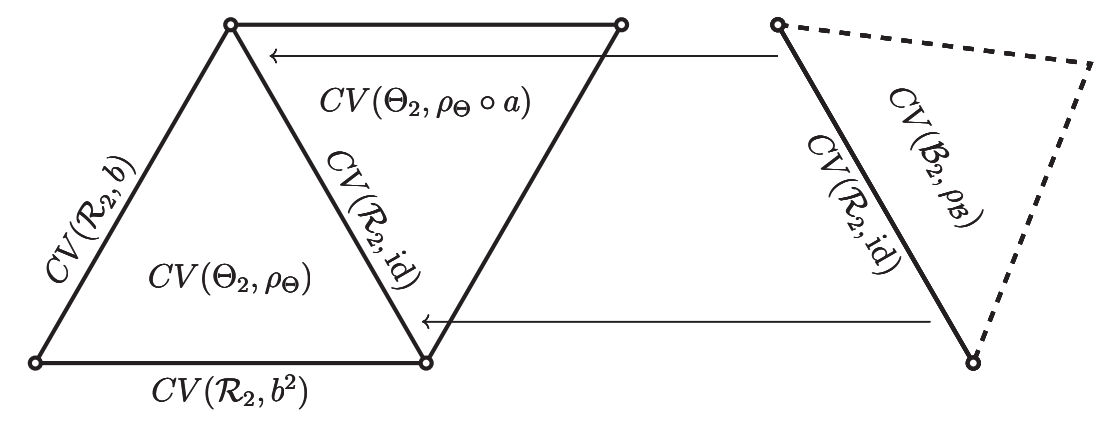

FIGURE 3. A portion of the Culler-Vogtmann outer space $C V\left(\mathbb{F}_{2}\right)$.

inverse to the collapse of $e_{1}$. Similarly, $\rho_{\mathcal{B}}$ is the homotopy inverse to the map $\mathcal{B}_{2} \rightarrow \mathcal{R}_{2}$ that collapses $e_{3}$.

One of the goals of this paper is to investigate a different continuous section of the quotient map. This uses the notion of entropy, $\mathfrak{h}_{G}(\ell)$, defined in $\S 3.1$. Using this notion, there is a section $\mathcal{H}: \mathbb{P} \mathcal{X}\left(\mathbb{F}_{r}\right) \rightarrow \mathcal{X}\left(\mathbb{F}_{r}\right)$ defined by

$$
\mathcal{H}([[(G, \rho, \ell)]])=\left[\left(G, \rho, \mathfrak{h}_{G}(\ell) \ell\right)\right] .
$$

We will denote the image of this section by $\mathcal{X}^{1}\left(\mathbb{F}_{r}\right)$.

\section{Thermodynamic metrics}

In this section we introduce the entropy and pressure of a length function in $\mathcal{M}(G)$, for a graph $G$ as in $\$ 2.1$. By normalizing the entropy to be equal to 1 , we realize $\mathcal{X}^{1}\left(\mathbb{F}_{r}\right)$ (as defined in $\S 2.2$ ) as a section of $\mathcal{X}\left(\mathbb{F}_{r}\right) \rightarrow \mathbb{P} \mathcal{X}\left(\mathbb{F}_{r}\right)$; it will follow that $\mathcal{X}^{1}\left(\mathbb{F}_{r}\right)$ is homeomorphic to $C V\left(\mathbb{F}_{r}\right)$ (see Theorem 3.5). We use entropy and pressure to construct piecewise Riemannian metrics on $\mathcal{X}^{1}\left(\mathbb{F}_{r}\right)$, which we call the thermodynamic metrics. Pollicott and Sharp were the first to consider one of these metrics [31]. Kao [22] and $\mathrm{Xu}[39]$ have also investigated these metrics. In these papers, the metric is only considered for a single marked graph and never on the entire outer space, as we will do here.

3.1. Entropy. Fix a finite connected graph $G=\left(V, E, o, \tau,{ }^{-}\right)$. An edge path $\left(e_{1}, \ldots, e_{n}\right)$ in a graph $G$ is reduced if $e_{i} \neq \bar{e}_{i+1}$ for $i=1, \ldots, n-1$. A reduced edge path $\left(e_{1}, \ldots, e_{n}\right)$ is a based circuit if $\tau\left(e_{n}\right)=o\left(e_{1}\right)$ and $e_{n} \neq \bar{e}_{1}$. The set of all based 
circuits in $G$ is denoted by $C(G)$. For a length function $\ell \in \mathcal{M}(G)$ and a real number $t \geq 0$, define

$$
C_{G, \ell}(t)=\{\gamma \in C(G) \mid \ell(\gamma) \leq t\} .
$$

Definition 3.1. The entropy of a length function $\ell \in \mathcal{M}(G)$ is

$$
\mathfrak{h}_{G}(\ell)=\lim _{t \rightarrow \infty} \frac{1}{t} \log \left|C_{G, \ell}(t)\right| .
$$

Remark 3.2. We defined entropy as the growth rate of the number of reduced based circuits. In the literature, there exist many equivalent definitions of entropy. In particular, one can count the growth of reduced edge paths in $G$ starting at a particular vertex and the adjective 'based' can be removed from the count of circuits [24, Proposition 2.3]. This shows that $\mathfrak{h}_{G}(\ell)$ equals the volume entropy of $(G, \ell)$. The volume entropy is defined as the exponential growth rate of the volume of balls in $\left(\widetilde{G}, g_{\ell}\right)$, where $\widetilde{G}$ is the universal cover of $G$ and $g_{\ell}$ is the piecewise Riemannian metric obtained by pulling back the length function $\ell$. That is,

$$
\mathfrak{h}_{G}(\ell)=\lim _{t \rightarrow \infty} \frac{1}{t} \log \operatorname{vol}_{g_{\ell}} B(x, t)
$$

where $B(x, t)$ is the ball of radius $t$ centered at $x \in \widetilde{G}$, which is an arbitrary basepoint.

Example 3.3. The number of reduced edge paths in $\mathcal{R}_{r}$ with 1-length equal to $n$ is exactly $2 r(2 r-1)^{n-1}$. Thus for any vertex $v \in \widetilde{\mathcal{R}}_{r}$ we have

$$
\operatorname{vol}_{g_{1}} B(v, n)=\frac{r}{r-1}\left((2 r-1)^{n}-1\right) .
$$

Hence $\mathfrak{h}_{\mathcal{R}_{r}}(\mathbf{1})=\log (2 r-1)$.

The next lemma shows that entropy is homogeneous of degree -1 and thus any length function $\ell \in \mathcal{M}(G)$ can be scaled to have unit entropy. Specifically, $\mathfrak{h}_{G}(a \cdot \ell)=1$ if and only if $a=\mathfrak{h}_{G}(\ell)$.

LEMMA 3.4. Let $G$ be a finite connected graph and fix $\ell \in \mathcal{M}(G)$. If $a \in \mathbb{R}_{>0}$, then

$$
\mathfrak{h}_{G}(a \cdot \ell)=\frac{1}{a} \mathfrak{h}_{G}(\ell)
$$

Proof. We reparametrize the limit defining entropy by setting $s=a t$. Then

$$
\begin{aligned}
\mathfrak{h}_{G}(\ell) & =\lim _{t \rightarrow \infty} \frac{1}{t} \log |\{\gamma \in C(G) \mid \ell(\gamma) \leq t\}| \\
& =\lim _{s \rightarrow \infty} \frac{a}{s} \log |\{\gamma \in C(G) \mid a \cdot \ell(\gamma) \leq s\}|=a \mathfrak{h}_{G}(a \cdot \ell) .
\end{aligned}
$$

Entropy defines an $\operatorname{Out}\left(\mathbb{F}_{r}\right)$-invariant function on $\mathcal{X}\left(\mathbb{F}_{r}\right)$ by $\mathfrak{h}([(G, \rho, \ell)])=\mathfrak{h}_{G}(\ell)$. This function was investigated by Kapovich and Nagnibeda, who showed the following theorem.

Theorem 3.5. [23, Theorem A] The entropy function $\mathfrak{h}: \mathcal{X}\left(\mathbb{F}_{r}\right) \rightarrow \mathbb{R}$ is continuous. 
In particular, the map $\mathcal{H}: \mathbb{P} X\left(\mathbb{F}_{r}\right) \rightarrow \mathcal{X}\left(\mathbb{F}_{r}\right)$ defined by normalizing to have unit entropy,

$$
\mathcal{H}([[(G, \rho, \ell)]])=\left[\left(G, \rho, \mathfrak{h}_{G}(\ell) \ell\right)\right],
$$

is a section. Hence the image $\mathcal{X}^{1}\left(\mathbb{F}_{r}\right)=\left\{[(G, \rho, \ell)] \in \mathcal{X}\left(\mathbb{F}_{r}\right) \mid \mathfrak{h}_{G}(\ell)=1\right\}$ is homeomorphic to $C V\left(\mathbb{F}_{r}\right)$.

3.2. Pressure. Fix a finite connected graph $G=\left(V, E, o, \tau,{ }^{-}\right)$. We assume throughout this subsection and the next that $\chi(G)<0$ (where $\chi(G)=|V|-\frac{1}{2}|E|$ is the Euler characteristic of $G$-note the $\frac{1}{2}$ factor is present as $E$ includes edges with both orientations) and that $G$ has no vertices with valence equal to 1 or 2 .

Define $A_{G} \in \operatorname{Mat}_{|E|}(\mathbb{R})$ by

$$
A_{G}\left(e, e^{\prime}\right)= \begin{cases}1 & \text { if } \tau(e)=o\left(e^{\prime}\right) \text { and } \bar{e} \neq e^{\prime} \\ 0 & \text { otherwise }\end{cases}
$$

It follows that the entry $A_{G}^{n}\left(e, e^{\prime}\right)$ is the number of reduced edge paths of the form $\left(e_{1}, \ldots, e_{n}\right)$ where $e_{1}=e, \tau\left(e_{n}\right)=o\left(e^{\prime}\right)$ and $\bar{e}_{n} \neq e^{\prime}$. In particular, $\operatorname{tr}\left(A_{G}^{n}\right)$ is the number of based edge circuits with 1-length equal to $n$. Denoting the spectral radius of a matrix by $\operatorname{spec}(\cdot)$, we get, from the definition of entropy, that

$$
\mathfrak{h}_{G}(\mathbf{1})=\log \left(\operatorname{spec}\left(A_{G}\right)\right) \text {. }
$$

We remark that the above assumptions on $G$ ensure that $A_{G}$ is irreducible.

In order to get a matrix that incorporates the metric and is related to entropy, we scale the rows of $A_{G}$ as follows: given a function $f: E \rightarrow \mathbb{R}$, we define $A_{G, f} \in \operatorname{Mat}_{|E|}(\mathbb{R})$ by

$$
A_{G, f}\left(e, e^{\prime}\right)=A_{G}\left(e, e^{\prime}\right) \exp (-f(e)) .
$$

As for $A_{G}$, it follows that $A_{G, f}^{n}\left(e, e^{\prime}\right)$ is the sum of $\exp (-f(\gamma))$ over all edge paths of the form $\gamma=\left(e_{1}, \ldots, e_{n}\right)$ where $e_{1}=e, \tau\left(e_{n}\right)=o\left(e^{\prime}\right)$ and $\bar{e}_{n} \neq e^{\prime}$.

Definition 3.6. The pressure of a function $f: E \rightarrow \mathbb{R}$ is defined as $\mathfrak{P}_{G}(f)=$ $\log \operatorname{spec}\left(A_{G,-f}\right)$.

By equation (3.2) we have that $\mathfrak{P}_{G}(\mathbf{0})=\mathfrak{h}_{G}(\mathbf{1})$ as $A_{G,-\mathbf{0}}=A_{G}$, where $\mathbf{0}$ is the zero function.

The connection between entropy and pressure is given by the following theorem.

THEOREM 3.7. Suppose that $G=\left(V, E, o, \tau,{ }^{-}\right)$is a finite connected graph. Then the following statements hold.

(1) For any length function $\ell \in \mathcal{M}(G), \mathfrak{P}_{G}(-\ell)=0$ if and only if $\mathfrak{h}_{G}(\ell)=1$.

(2) The pressure function $\mathfrak{P}_{G}: \mathbb{R}^{\left|E_{+}\right|} \rightarrow \mathbb{R}$ is real analytic and convex.

(3) The entropy function $\mathfrak{h}_{G}: \mathcal{M}(G) \rightarrow \mathbb{R}$ is real analytic and strictly convex.

Proof. The first item appears in the work of Pollicott and Sharp [31, Lemma 3.1(2)].

The properties of pressure stated in the second item can be found in the work of Parry and Pollicott [29, Propositions 4.7 and 4.12]. 
The properties of entropy stated in the third item can be found in the work of McMullen [28, Proposition A.4]. Kapovich and Nagnibeda gave an alternative proof of the real analyticity of $\mathfrak{h}_{G}[23]$.

Let $\mathcal{M}^{1}(G)=\left\{\ell \in \mathcal{M}(G) \mid \mathfrak{h}_{G}(\ell)=1\right\}$. By the first item above, we have that alternatively $\mathcal{M}^{1}(G)=\left\{\ell \in \mathcal{M}(G) \mid \mathfrak{P}_{G}(-\ell)=0\right\}$. To see that $\mathcal{M}^{1}(G)$ is a codimension-1 submanifold of $\mathbb{R}^{\left|E_{+}\right|}$we need to argue that 1 is a regular value of $\mathfrak{h}_{G}$. This follows from the following lemma. We denote the standard Euclidean inner product on $\mathbb{R}^{n}$ by $\langle\cdot, \cdot\rangle$.

LEMMA 3.8. Let $G$ be a finite connected graph and fix $\ell \in \mathcal{M}(G)$. Then $\left\langle\ell, \nabla \mathfrak{h}_{G}(\ell)\right\rangle=$ $-\mathfrak{h}_{G}(\ell)$.

Proof. This follows from the homogeneity of the entropy function (Lemma 3.4). Indeed,

$$
\begin{aligned}
\left\langle\ell, \nabla \mathfrak{h}_{G}(\ell)\right\rangle & =\lim _{s \rightarrow 0} \frac{\mathfrak{h}_{G}(\ell+s \ell)-\mathfrak{h}_{G}(\ell)}{s}=\lim _{s \rightarrow 0} \frac{\mathfrak{h}_{G}((1+s) \ell)-\mathfrak{h}_{G}(\ell)}{s} \\
& =\lim _{s \rightarrow 0} \frac{\frac{1}{1+s} \mathfrak{h}_{G}(\ell)-\mathfrak{h}_{G}(\ell)}{s}=\mathfrak{h}_{G}(\ell) \lim _{s \rightarrow 0} \frac{-s}{s(s+1)}=-\mathfrak{h}_{G}(\ell) .
\end{aligned}
$$

We record the following properties of the partial derivatives and the gradient of the pressure function. Given a function $f: \mathbb{R}^{\left|E_{+}\right|} \rightarrow \mathbb{R}$ and an edge $e \in E_{+}$, we denote the partial derivative of $f$ with respect to the $e$ th coordinate by $\partial_{e} f$. Let $\|\cdot\|_{1}$ denote the usual $L^{1}$-norm on vectors in $\mathbb{R}^{n}$.

LEMMA 3.9. Let $G=\left(V, E, o, \tau,{ }^{-}\right)$be a finite connected graph and fix $\ell \in \mathcal{M}(G)$. Then the following statements hold.

(1) $\partial_{e} \mathfrak{P}_{G}(\ell)>0$ for any $e \in E_{+}$.

(2) $\left\|\nabla \mathfrak{P}_{G}(\ell)\right\|_{1}=1$.

Proof. By the Perron-Frobenius theorem, the spectral radius of $A_{G, \ell}$ is realized by a positive, real, simple eigenvalue $\lambda$. Let $\mathbf{v} \in \mathbb{R}^{|E|}$ be a corresponding positive left eigenvector so that $\mathbf{v} A_{G, \ell}=\lambda \mathbf{v}$. Consider the matrix $Q_{G, \ell} \in \operatorname{Mat}_{|E|}(\mathbb{R})$ defined by $Q_{G, \ell}\left(e, e^{\prime}\right)=\left(\mathbf{v}(e) / \lambda \mathbf{v}\left(e^{\prime}\right)\right) A_{G, \ell}\left(e, e^{\prime}\right)$. Again, by the Perron-Frobenius theorem, as this matrix is column stochastic, there is a positive vector $\mathbf{p} \in \mathbb{R}^{|E|}$ with $Q_{G, \ell} \mathbf{p}=\mathbf{p}$ and $\|\mathbf{p}\|_{1}=1$. As explained by Parry and Pollicott, we have that $\partial_{e} \mathfrak{P}_{G}(\ell)=\mathbf{p}(e)+\mathbf{p}(\bar{e})[29$, Ch. 2, Remark 1 and Proposition 4.10]. Items (3.9) and (3.9) readily follow.

3.3. Thermodynamic metrics. Fix a finite connected graph $G=\left(V, E, o, \tau,{ }^{-}\right)$. As in the previous section, we assume that $\chi(G)<0$ and that $G$ has no vertices with valence equal to 1 or 2 . The tangent space $T_{\ell} \mathcal{M}^{1}(G)$ at the length function $\ell \in \mathcal{M}^{1}(G)$ is the space of vectors $\mathbf{v} \in \mathbb{R}^{\left|E_{+}\right|}$such that $\left\langle\mathbf{v}, \nabla \mathfrak{h}_{G}(\ell)\right\rangle=0$. The tangent bundle $T \mathcal{M}^{1}(G)$ is the subspace of $\mathcal{M}^{1}(G) \times \mathbb{R}^{\left|E_{+}\right|}$consisting of pairs $(\ell, \mathbf{v})$ where $\mathbf{v} \in T_{\ell} \mathcal{M}^{1}(G)$.

We now define two Riemannian metrics on $\mathcal{M}^{1}(G)$. We denote the Hessian (that is, the matrix of second derivatives) of a smooth function $f: \mathbb{R}^{n} \rightarrow \mathbb{R}$ by $\mathbf{H}[f(x)]$. 
Definition 3.10. Given a length function $\ell \in \mathcal{M}^{1}(G)$ and tangent vectors $\mathbf{v}_{1}, \mathbf{v}_{2} \in$ $T_{\ell} \mathcal{M}^{1}(G)$ we define the entropy metric by

$$
\left\langle\mathbf{v}_{1}, \mathbf{v}_{2}\right\rangle_{\mathfrak{h}, G}=\left\langle\mathbf{v}_{1}, \mathbf{H}\left[\mathfrak{h}_{G}(\ell)\right] \mathbf{v}_{2}\right\rangle,
$$

and the pressure metric by

$$
\left\langle\mathbf{v}_{1}, \mathbf{v}_{2}\right\rangle_{\mathfrak{P}, G}=\left\langle\mathbf{v}_{1}, \mathbf{H}\left[\mathfrak{P}_{G}(-\ell)\right] \mathbf{v}_{2}\right\rangle .
$$

The associated norms on the tangent bundle $T \mathcal{M}^{1}(G)$ are denoted by

$$
\|(\ell, \mathbf{v})\|_{\mathfrak{h}, G}^{2}=\left\langle\mathbf{v}, \mathbf{H}\left[\mathfrak{h}_{G}(\ell)\right] \mathbf{v}\right\rangle \quad \text { and } \quad\|(\ell, \mathbf{v})\|_{\mathfrak{P}, G}^{2}=\left\langle\mathbf{v}, \mathbf{H}\left[\mathfrak{P}_{G}(-\ell)\right] \mathbf{v}\right\rangle \text {. }
$$

By Theorem 3.7(3) we have that $\langle\cdot, \cdot\rangle_{\mathfrak{h}, G}$ is positive definite. Positive definiteness of $\langle\cdot, \cdot\rangle_{\mathfrak{P}, G}$ on $T_{\ell} \mathcal{M}^{1}(G)$ has been noted by others, but also follows from the positive definiteness of $\langle\cdot, \cdot\rangle_{\mathfrak{h}, G}$ by Proposition 3.12.

Remark 3.11. Other authors have considered these metrics with different and conflicting terminology. We discuss this now using the notation introduced above. Pollicott and Sharp defined $\|\cdot\|_{\mathfrak{P}, G}$, calling it the Weil-Petersson metric [31]. Kao defined $\|\cdot\|_{\mathfrak{h}, G}$, calling it the Weil-Petersson metric, and also studied $\|\cdot\|_{\mathfrak{P}, G}$, calling it the pressure metric [22]. Xu considered $\|\cdot\|_{\mathfrak{h}, G}$, calling it the pressure metric [39]. We use the terminology as stated in Definition 3.10 as it accurately reflects the functions on which the metrics are based. The definitions of these metrics in the literature are not those as given in Definition 3.10, but are equivalent as can be seen by Proposition 3.12.

We note that Theorem 3 in the paper by Pollicott and Sharp [31] holds for the metric $\|\cdot\|_{\mathfrak{h}, G}$ and not for $\|\cdot\|_{\mathfrak{P}, G}$ as claimed.

The following proposition shows that these metrics lie in the same conformal class and that they can be calculated using the second derivative along a path. This feature is essential particularly for the material in $\S 10$.

Proposition 3.12. Let $G$ be a finite connected graph. If $\ell_{t}:(-1,1) \rightarrow \mathcal{M}^{1}(G)$ is a smooth path, then

$$
\left\|\left(\ell_{t}, \dot{\ell}_{t}\right)\right\|_{\mathfrak{h}, G}^{2}=-\left\langle\ddot{\ell}_{t}, \nabla \mathfrak{h}_{G}\left(\ell_{t}\right)\right\rangle \quad \text { and }\left\|\left(\ell_{t}, \dot{\ell}_{t}\right)\right\|_{\mathfrak{P}, G}^{2}=\left\langle\ddot{\ell}_{t}, \nabla \mathfrak{P}_{G}\left(-\ell_{t}\right)\right\rangle .
$$

Additionally, given a length function $\ell \in \mathcal{M}^{1}(G)$ and tangent vectors $\mathbf{v}_{1}, \mathbf{v}_{2} \in T_{\ell} \mathcal{M}^{1}(G)$, we have

$$
\left\langle\mathbf{v}_{1}, \mathbf{v}_{2}\right\rangle_{\mathfrak{h}, G}=\frac{\left\langle\mathbf{v}_{1}, \mathbf{v}_{2}\right\rangle_{\mathfrak{P}, G}}{\left\langle\ell, \nabla \mathfrak{P}_{G}(-\ell)\right\rangle} .
$$

Proof. Differentiating the equation $\mathfrak{P}_{G}\left(-\ell_{t}\right)=0$ with respect to $t$, we have $\left\langle\dot{\ell}_{t}\right.$, $\left.\nabla \mathfrak{P}_{G}\left(-\ell_{t}\right)\right\rangle=0$. Differentiating again, we find that

$$
\left\langle\ddot{\ell}_{t}, \nabla \mathfrak{P}_{G}\left(-\ell_{t}\right)\right\rangle-\left\langle\dot{\ell}_{t}, \mathbf{H}\left[\mathfrak{P}_{G}\left(-\ell_{t}\right)\right] \dot{\ell}_{t}\right\rangle=0 .
$$

Hence $\left\|\left(\ell_{t}, \dot{\ell}_{t}\right)\right\|_{\mathfrak{P}}^{2}=\left\langle\dot{\ell}_{t}, \mathbf{H}\left[\mathfrak{P}\left(-\ell_{t}\right)\right] \dot{\ell}_{t}\right\rangle=\left\langle\ddot{\ell}_{t}, \nabla \mathfrak{P}\left(-\ell_{t}\right)\right\rangle$ as claimed.

The proof of the analogous statement for the entropy norm is similar, observing that $\mathfrak{h}_{G}\left(\ell_{t}\right)=1$. 
By Lemma 3.8, we have that $\left\langle\ell, \nabla \mathfrak{h}_{G}(\ell)\right\rangle=-1$ for any $\ell \in \mathcal{M}^{1}(G)$. Further, we have that $\nabla \mathfrak{P}_{G}(-\ell)$ is non-zero by Lemma 3.9 and hence is parallel to $\nabla \mathfrak{h}_{G}(\ell)$ by Theorem 3.7(1). Hence we find that

$$
\left\|\left(\ell_{t}, \dot{\ell}_{t}\right)\right\|_{\mathfrak{h}, G}^{2}=-\left\langle\ddot{\ell}_{t}, \nabla \mathfrak{h}_{G}\left(\ell_{t}\right)\right\rangle=\frac{\left\langle\ddot{\ell}_{t}, \nabla \mathfrak{h}_{G}\left(\ell_{t}\right)\right\rangle}{\left\langle\ell_{t}, \nabla \mathfrak{h}_{G}\left(\ell_{t}\right)\right\rangle}=\frac{\left\langle\ddot{\ell}_{t}, \nabla \mathfrak{P}_{G}\left(-\ell_{t}\right)\right\rangle}{\left\langle\ell_{t}, \nabla \mathfrak{P}_{G}\left(-\ell_{t}\right)\right\rangle}=\frac{\left\|\left(\ell_{t}, \dot{\ell}_{t}\right)\right\|_{\mathfrak{P}, G}^{2}}{\left\langle\ell_{t}, \nabla \mathfrak{P}_{G}\left(-\ell_{t}\right)\right\rangle} .
$$

By polarization, the norm determines the inner product and so the claim follows.

Positive definiteness of the Hessians follows from strict convexity of $\mathfrak{h}_{G}$ and $\mathfrak{P}_{G}$ on $\mathcal{M}(G)$ and $\mathbb{R}^{\left|E_{+}\right|}$, respectively (Theorem 3.7).

Using these norms, we can define the entropy or pressure length of a piecewise smooth path $\ell_{t}:\left[t_{0}, t_{1}\right] \rightarrow \mathcal{M}^{1}(G)$ by

$$
\begin{aligned}
\mathcal{L}_{\mathfrak{h}, G}\left(\ell_{t} \mid\left[t_{0}, t_{1}\right]\right) & =\int_{t_{0}}^{t_{1}}\left\|\left(\ell_{t}, \dot{\ell}_{t}\right)\right\|_{\mathfrak{h}, G} d t, \\
\mathcal{L}_{\mathfrak{P}, G}\left(\ell_{t} \mid\left[t_{0}, t_{1}\right]\right) & =\int_{t_{0}}^{t_{1}}\left\|\left(\ell_{t}, \dot{\ell}_{t}\right)\right\|_{\mathfrak{P}, G} d t .
\end{aligned}
$$

These induce the entropy and pressure distance functions on $\mathcal{M}^{1}(G)$ by

$$
\begin{aligned}
d_{\mathfrak{h}, G}(x, y) & =\inf \left\{\mathcal{L}_{\mathfrak{h}, G}\left(\ell_{t} \mid[0,1]\right) \mid \ell_{t}:[0,1] \rightarrow \mathcal{M}^{1}(G), \ell_{0}=x, \ell_{1}=y\right\}, \\
d_{\mathfrak{P}, G}(x, y) & =\inf \left\{\mathcal{L}_{\mathfrak{P}, G}\left(\ell_{t} \mid[0,1]\right) \mid \ell_{t}:[0,1] \rightarrow \mathcal{M}^{1}(G), \ell_{0}=x, \ell_{1}=y\right\} .
\end{aligned}
$$

Given a marked graph $(G, \rho)$, we set $\mathcal{X}^{1}(G, \rho)=\mathcal{X}(G, \rho) \cap \mathcal{X}^{1}\left(\mathbb{F}_{r}\right)$. Using the natural bijection $\mathcal{X}^{1}(G, \rho) \leftrightarrow \mathcal{M}^{1}(G)$, we get metrics and distance functions on $\mathcal{X}^{1}(G, \rho)$ that we denote using the same notation as above.

Next, we explain how these fit together to get distance functions on $\mathcal{X}^{1}\left(\mathbb{F}_{r}\right)$. Suppose $\alpha_{t}:[0,1] \rightarrow \mathcal{X}^{1}\left(\mathbb{F}_{r}\right)$ is a piecewise smooth path and that there is a partition $t_{1}=0<t_{2}<$ $\cdots<t_{n+1}=1$ and marked graphs $\left(G_{k}, \rho_{k}\right)$ for $k=1, \ldots, n$ such that $\alpha_{t} \in \mathcal{X}^{1}\left(G_{k}, \rho_{k}\right)$ for $t \in\left(t_{k}, t_{k+1}\right)$. We set

$$
\mathcal{L}_{\mathfrak{h}}\left(\alpha_{t}\right)=\sum_{k=1}^{n} \mathcal{L}_{\mathfrak{h}, G_{k}}\left(\alpha_{t} \mid\left(t_{k}, t_{k+1}\right)\right) \quad \text { and } \quad \mathcal{L}_{\mathfrak{P}}\left(\alpha_{t}\right)=\sum_{k=1}^{n} \mathcal{L}_{\mathfrak{P}, G_{k}}\left(\left(\alpha_{t} \mid\left(t_{k}, t_{k+1}\right)\right) .\right.
$$

These define distance functions on $\mathcal{X}^{1}\left(\mathbb{F}_{r}\right)$ as usual—by taking the infimum of the lengths of paths - that we denote by $d_{\mathfrak{h}}$ and $d_{\mathfrak{P}}$. It is obvious that the proposed distance functions are symmetric and satisfy the triangle inequality; positive definiteness of the Hessians implies non-degeneracy. However, it is not obvious that the distances they define are finite: a priori it may be possible that the length of a path that collapses an edge is infinite. This will be addressed in $\S 5$.

Remark 3.13. For the remainder of the paper we will mainly be concerned with the entropy metric. As stated in $\S 1.6$, the main results of this paper also hold for the pressure metric with slightly altered hypotheses. 
4. A determinant-defining equation for $\mathcal{M}^{1}(G)$

The purpose of this section is to derive formulas to assist in computing the metrics introduced in the previous section. The first of these appears in $\S 4.1$, specifically Proposition 4.6, where it is shown that these metrics can be computed using finite sums of exponential functions on $\mathcal{M}^{1}(G)$. Next, in $\S 4.2$, we present a simplification for certain graphs that is useful in the sequel.

4.1. Determinant equation. Fix a finite connected graph $G=\left(V, E, o, \tau,{ }^{-}\right)$. We always assume that $\chi(G)<0$ and $G$ has no vertices of valence 1 or 2 .

Let $D_{G}$ be the directed graph with adjacency matrix $A_{G}$. Thus the vertex set for $D_{G}$ is the set $E$ (recall our notation $E=E_{+} \cup \bar{E}_{+}$) and there is an edge from $e$ to $e^{\prime}$ if $A_{G}\left(e, e^{\prime}\right)=1$, that is, if $\tau(e)=o\left(e^{\prime}\right)$ and $\bar{e} \neq e^{\prime}$. The cycle complex of $D_{G}$, denoted by $C_{G}$, is the abstract simplicial complex with an $n$-simplex for each collection $\Delta=$ $\left\{z_{1}, \ldots, z_{n+1}\right\}$ of pairwise disjoint simple cycles, that is, embedded loops, in $D_{G}$.

Example 4.1. Suppose that $G$ is the barbell graph as shown below:

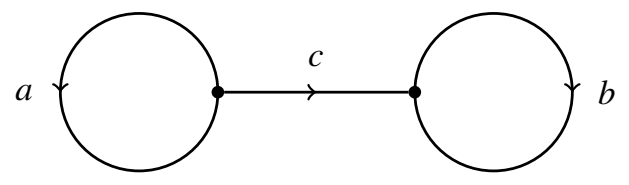

Order the edges of $G$ by $a, \bar{a}, b, \bar{b}, c, \bar{c}$. The matrix $A_{G}$ and directed graph $D_{G}$ are as presented below:

$$
\left[\begin{array}{llllll}
1 & 0 & 0 & 0 & 1 & 0 \\
0 & 1 & 0 & 0 & 1 & 0 \\
0 & 0 & 1 & 0 & 0 & 1 \\
0 & 0 & 0 & 1 & 0 & 1 \\
0 & 0 & 1 & 1 & 0 & 0 \\
1 & 1 & 0 & 0 & 0 & 0
\end{array}\right]
$$

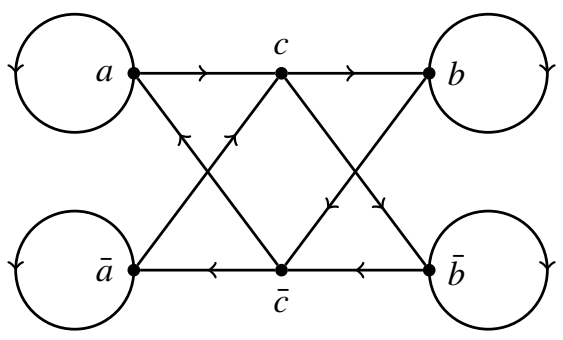

There are eight simple cycles in $D_{G}: \gamma_{a}=(a), \gamma_{\bar{a}}=(\bar{a}), \gamma_{b}=(b), \gamma_{\bar{b}}=(\bar{b}), \gamma_{a b}=$ $(a, c, b, \bar{c}), \gamma_{a \bar{b}}=(a, c, \bar{b}, \bar{c}), \gamma_{\bar{a} b}=(\bar{a}, c, b, \bar{c})$ and $\gamma_{\bar{a} \bar{b}}=(\bar{a}, c, \bar{b}, \bar{c})$. The cycle complex $C_{G}$ is the flag complex whose 1-skeleton is shown in Figure 4.

Given a function $f: E \rightarrow \mathbb{R}$ (in particular, a length function) and a simple cycle $z$ in $D_{G}$, we set $f(z)=\sum_{i=1}^{n} f\left(e_{i}\right)$ where $e_{1}, \ldots, e_{n}$ are the vertices in $D_{G}$ traversed by $z$ (each corresponding to an oriented edge in $G$ ). Likewise, for a simplex $\Delta=\left\{z_{1}, \ldots, z_{n}\right\}$ in $C_{G}$ we set $f(\Delta)=\sum_{k=1}^{n} f\left(z_{k}\right)$. We consider the empty set as a simplex and define $f(\emptyset)=0$ for any function $f: E \rightarrow \mathbb{R}$. Lastly, for a simplex $\Delta=\left\{z_{1}, \ldots, z_{n}\right\}$ we set $|\Delta|=n$.

Recall that given a length function $\ell \in \mathcal{M}(G)$, the matrix $A_{G, \ell}$ is defined by $A_{G, \ell}\left(e, e^{\prime}\right)=\exp (-\ell(e)) A_{G}\left(e, e^{\prime}\right)$. We consider the function $F_{G}: \mathcal{M}(G) \rightarrow \mathbb{R}$ 


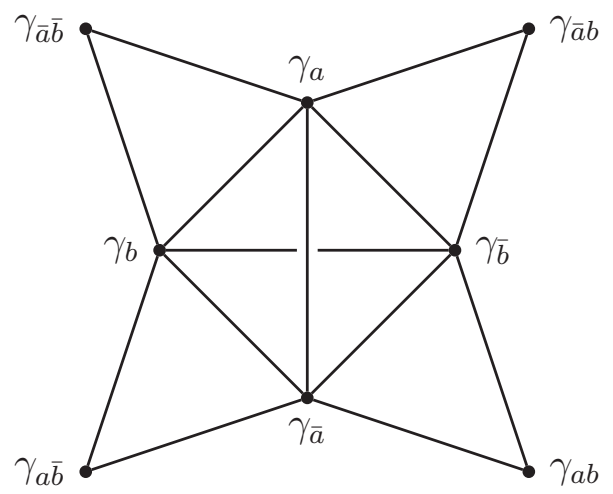

FIGURE 4. The 1-skeleton of the cycle complex $C_{G}$ in Example 4.1.

given by

$$
F_{G}(\ell)=\operatorname{det}\left(I-A_{G, \ell}\right) .
$$

This function can be expressed using the cycle complex $C_{G}$ as follows.

THEOREM 4.2. Let $G$ be a finite connected graph and fix $\ell \in \mathcal{M}(G)$. Then

$$
F_{G}(\ell)=\sum_{\Delta \in C_{G}}(-1)^{|\Delta|} \exp (-\ell(\Delta))
$$

Proof. This follows from the coefficient theorem for digraphs. See, for instance, [15] and [3, Theorems 2.5 and 2.14].

Example 4.3. We apply Theorem 4.2 to the case when $G$ is the barbell graph as in Example 4.1. Using the change of variables $x=\exp (-\ell(a)), y=\exp (-\ell(b))$ and $z=$ $\exp (-\ell(c))$, we find that

$$
\begin{aligned}
F_{G}(\ell)= & 1-\left(2 x+2 y+4 x y z^{2}\right)+\left(x^{2}+y^{2}+4 x y+4 x^{2} y z^{2}+4 x y^{2} z^{2}\right) \\
& -\left(2 x^{2} y+2 x y^{2}+4 x^{2} y^{2} z^{2}\right)+x^{2} y^{2} .
\end{aligned}
$$

The following statements show how the function $F_{G}$ is related to $\mathcal{M}^{1}(G)$.

LEMMA 4.4. For $\ell \in \mathcal{M}^{1}(G)$ we have:

(1) $F_{G}(\ell)=0$;

(2) $\nabla F_{G}(\ell) \neq 0$; and moreover,

(3) $\partial_{e} F_{G}(\ell)>0$ for any $e \in E_{+}$.

Proof. Since $\ell$ lies in $\mathcal{M}^{1}(G)$, Theorem 3.7(1) and the definition of pressure imply that $\operatorname{spec}\left(A_{G, \ell}\right)=1$. Above we remarked that the assumptions on $G$ imply that $A_{G}$ is irreducible; hence so is $A_{G, \ell}$. By the Perron-Frobenius theorem the spectral radius of $A_{G, \ell}$ is realized by a positive, real, simple eigenvalue; (1) follows. 
Now consider the function $p: \mathbb{R} \rightarrow \mathbb{R}$ defined by $p(t)=\operatorname{det}\left(I-t A_{G, \ell}\right)$. Let $1=$ $\lambda_{1}, \ldots, \lambda_{|E|}$ be the roots of the characteristic polynomial of $A_{G, \ell}$. Then we can write

$$
p(t)=(1-t) \prod_{i=2}^{|E|}\left(1-t \lambda_{i}\right) .
$$

Therefore, taking the derivative, we find that

$$
p^{\prime}(1)=-\prod_{i=2}^{|E|}\left(1-\lambda_{i}\right) .
$$

For $i=2, \ldots,|E|$ we have that $\left|\lambda_{i}\right| \leq 1$ and $\lambda_{i} \neq 1$. Combining these observations with the fact that complex eigenvalues come in conjugate pairs, it follows that $p^{\prime}(1)<0$. Observe that $F_{G}(\ell+s \cdot \mathbf{1})=\operatorname{det}\left(I-\exp (-s) A_{G, \ell}\right)=p(\exp (-s))$. Therefore we have that $\left\langle\mathbf{1}, \nabla F_{G}(\ell)\right\rangle=-p^{\prime}(1) \exp (0)>0$, giving (2).

We claim that $\nabla F_{G}(\ell)$ is parallel to $\nabla \mathfrak{P}_{G}(\ell)$ for $\ell \in \mathcal{M}^{1}(G)$. Indeed, this follows as (1), (2) and Theorem 3.7 imply that $\mathfrak{P}_{G}^{-1}(0)=\mathcal{M}^{1}(G)$ is a connected component of $F_{G}^{-1}(0)$. By (2) and Lemma 3.9(2) the gradients of $F_{G}$ and $\mathfrak{P}_{G}$ are non-zero for this subset. As gradients are always orthogonal to level sets, the claim follows. Hence by Lemma 3.9(1), we have that either $\partial_{e} F_{G}(\ell)>0$ or $\partial_{e} F_{G}(\ell)<0$ for all $e \in E_{+}$and $\ell \in \mathcal{M}^{1}(G)$. Since $\left\langle\mathbf{1}, \nabla F_{G}(\ell)\right\rangle>0$, we must have the former, whence (3).

As a remarked in the proof of Lemma 4.4, we have the following corollary.

COROLLARY 4.5. The unit-entropy moduli space $\mathcal{M}^{1}(G)$ is a connected component of the level set $\left\{\ell \in \mathcal{M}(G) \mid F_{G}(\ell)=0\right\}$.

Using these observations, we can compute the entropy and pressure norms using the function $F_{G}$.

Proposition 4.6. If $\ell_{t}:(-1,1) \rightarrow \mathcal{M}^{1}(G)$ is a smooth path, then

$$
\begin{gathered}
\left\|\left(\ell_{t}, \dot{\ell}_{t}\right)\right\|_{\mathfrak{h}, G}^{2}=\frac{-\left\langle\dot{\ell}_{t}, \mathbf{H}\left[F_{G}\left(\ell_{t}\right)\right] \dot{\ell}_{t}\right\rangle}{\left\langle\ell_{t}, \nabla F_{G}\left(\ell_{t}\right)\right\rangle}=\frac{\left\langle\ddot{\ell}_{t}, \nabla F_{G}\left(\ell_{t}\right)\right\rangle}{\left\langle\ell_{t}, \nabla F_{G}\left(\ell_{t}\right)\right\rangle}, \\
\left\|\left(\ell_{t}, \dot{\ell}_{t}\right)\right\|_{\mathfrak{P}, G}^{2}=\frac{-\left\langle\dot{\ell}_{t}, \mathbf{H}\left[F_{G}\left(\ell_{t}\right)\right] \dot{\ell}_{t}\right\rangle}{\left\|\nabla F_{G}\left(\ell_{t}\right)\right\|_{1}}=\frac{\left\langle\ddot{\ell}_{t}, \nabla F_{G}\left(\ell_{t}\right)\right\rangle}{\left\|\nabla F_{G}\left(\ell_{t}\right)\right\|_{1}} .
\end{gathered}
$$

Proof. The proof of the formula for the entropy norm is similar to that of Proposition 3.12 and left to the reader.

The proof of the formula for the pressure norm is again similar, noting that $\|\nabla \mathfrak{P}(\ell)\|_{1}=1$ as stated in Lemma 3.9(2).

Using Theorem 4.2, we can compute the partial derivatives of $F_{G}$. We find for any edges $e, e^{\prime} \in E_{+}$that

$$
\begin{aligned}
\partial_{e} F_{G}(\ell) & =-\sum_{\Delta \in C_{G}}(-1)^{|\Delta|} \Delta(e) \exp (-\ell(\Delta)), \\
\partial_{e e^{\prime}} F_{G}(\ell) & =\sum_{\Delta \in C_{G}}(-1)^{|\Delta|} \Delta(e) \Delta\left(e^{\prime}\right) \exp (-\ell(\Delta)),
\end{aligned}
$$


where $\Delta(e) \in\{0,1,2\}$ denotes the cardinality of the intersection $\{e, \bar{e}\} \cap \Delta$. Using this notation, we remark that $\ell(\Delta)=\sum_{e \in E_{+}} \Delta(e) \ell(e)$ for a length function $\ell \in \mathcal{M}(G)$ and simplex $\Delta \in C_{G}$. Given a vector $\mathbf{v} \in \mathbb{R}^{\left|E_{+}\right|}$and a simplex $\Delta \in C_{G}$, we set $\mathbf{v}(\Delta)=$ $\sum_{e \in E_{+}} \Delta(e) \mathbf{v}(e)$. Using these expressions, we can rewrite the dot products appearing in the formulas for the metrics in Proposition 4.6 as sums over simplices in $C_{G}$ rather than over the edges of $G$ as follows.

LEMMA 4.7. For $\ell \in \mathcal{M}(G)$, we have

$$
\left\langle\ell, \nabla F_{G}(\ell)\right\rangle=-\sum_{\Delta \in C_{G}}(-1)^{|\Delta|} \ell(\Delta) \exp (-\ell(\Delta)) .
$$

Proof. We compute:

$$
\begin{aligned}
\left\langle\ell, \nabla F_{G}(\ell)\right\rangle & =\sum_{e \in E_{+}} \ell(e)\left(-\sum_{\Delta \in C_{G}}(-1)^{|\Delta|} \Delta(e) \exp (-\ell(\Delta))\right) \\
& =-\sum_{\Delta \in C_{G}}(-1)^{|\Delta|} \exp (-\ell(\Delta))\left(\sum_{e \in E_{+}} \ell(e) \Delta(e)\right) \\
& =-\sum_{\Delta \in C_{G}}(-1)^{|\Delta|} \ell(\Delta) \exp (-\ell(\Delta)) .
\end{aligned}
$$

LEMMA 4.8. Let $G$ be a finite connected graph. If $(\ell, \mathbf{v}) \in T \mathcal{M}^{1}(G)$, then

$$
\left\langle\mathbf{v}, \mathbf{H}\left[F_{G}(\ell)\right] \mathbf{v}\right\rangle=\sum_{\Delta \in C_{G}}(-1)^{|\Delta|} \mathbf{v}(\Delta)^{2} \exp (-\ell(\Delta)) .
$$

Proof. This is similar to Lemma 4.7. The eth component of $\mathbf{H}\left[F_{G}(\ell)\right] \mathbf{v}$ is

$$
\sum_{e^{\prime} \in E_{+}}\left(\sum_{\Delta \in C_{G}}(-1)^{|\Delta|} \Delta(e) \Delta\left(e^{\prime}\right) \exp (-\ell(\Delta))\right) \mathbf{v}\left(e^{\prime}\right)=\sum_{\Delta \in C_{G}}(-1)^{|\Delta|} \Delta(e) \mathbf{v}(\Delta) \exp (-\ell(\Delta)) \text {. }
$$

Hence

$$
\begin{aligned}
\left\langle\mathbf{v}, \mathbf{H}\left[\nabla F_{G}(\ell)\right] \mathbf{v}\right\rangle & =\sum_{e \in E_{+}} \mathbf{v}(e)\left(\sum_{\Delta \in C_{G}}(-1)^{|\Delta|} \Delta(e) \mathbf{v}(\Delta) \exp (-\ell(\Delta))\right) \\
& =\sum_{\Delta \in C_{G}}(-1)^{|\Delta|} \mathbf{v}(\Delta)^{2} \exp (-\ell(\Delta)) .
\end{aligned}
$$

By Lemma 4.4, if $\mathfrak{h}_{G}(\ell)=1$ then $F_{G}(\ell)=0$. The next lemma gives a partial converse.

LEMMA 4.9. If $\mathfrak{h}_{G}(\ell)<1$, then $F_{G}(\ell)>0$.

Proof. We begin by showing that if $\mathfrak{h}_{G}(\ell)<1$, then $F_{G}(\ell) \neq 0$. To begin, we observe that if $\mathfrak{h}_{G}(\ell)<1$, then $\mathfrak{P}_{G}(-\ell)<0$. Indeed, let $\ell_{t}:[0,1] \rightarrow \mathcal{M}(G)$ be the path defined by

$$
\ell_{t}=(1-t) \ell+t \mathfrak{h}_{G}(\ell) \ell .
$$

We have that $\left\langle\nabla \mathfrak{P}_{G}\left(-\ell_{t}\right),-\dot{\ell}_{t}\right\rangle>0$ as each component of $\nabla \mathfrak{P}_{G}\left(-\ell_{t}\right)$ is positive by Lemma 3.9(1) and each component of $-\dot{\ell}_{t}$ is positive by construction. Notice that 
$\mathfrak{P}_{G}\left(-\ell_{1}\right)=0$ by Theorem 3.7(1) since $\mathfrak{h}_{G}\left(\ell_{1}\right)=1$. Therefore, we find that

$$
-\mathfrak{P}_{G}\left(-\ell_{0}\right)=\int_{0}^{1}\left\langle\nabla \mathfrak{P}_{G}\left(-\ell_{t}\right),-\dot{\ell}_{t}\right\rangle d t>0
$$

and hence $\mathfrak{P}_{G}(-\ell)=\mathfrak{P}_{G}\left(-\ell_{0}\right)$ is negative as claimed. Therefore we have that $\operatorname{spec}\left(A_{G, \ell}\right)<1$ and, in particular, 1 is not an eigenvalue of $A_{G, \ell}$. Hence $F_{G}(\ell) \neq 0$. This completes the claim that $F_{G}(\ell) \neq 0$ for any $\ell \in \mathcal{M}(G)$ where $\mathfrak{h}_{G}(\ell)<1$.

We now complete the proof of the lemma. Suppose that $\mathfrak{h}_{G}(\ell)<1$ and consider the continuous function $p:[0, \infty) \rightarrow \mathbb{R}$ defined by $p(t)=F_{G}(\ell+t \cdot \mathbf{1})$. As $\mathfrak{h}_{G}(\ell+t \cdot \mathbf{1})<1$ for all $t \in[0, \infty)$, by the above claim we have that $p(t) \neq 0$. Since $p(t) \rightarrow 1$ as $t \rightarrow \infty$, we have that $p(t)>0$ for all $t \in[0, \infty)$. In particular, we have that $F_{G}(\ell)=p(0)>0$.

4.2. A simplification. The function $F_{G}$ factors non-trivially in special cases as a result of certain aspects of the graph $G$. In such a case, we can replace $F_{G}$ with one of these factors and simplify the expressions for the entropy and pressure norm from Proposition 4.6.

For instance, one factorization occurs if $e$ is a loop edge. When $\ell(e)=0$ the vector $\mathbf{v} \in \mathbb{R}^{|E|}$, where $\mathbf{v}(e)=1, \mathbf{v}(\bar{e})=-1$, and the rest of the entries are equal to 0 , is an eigenvector of $A_{G, \ell}$ with eigenvalue 1 . This means $1-\exp (-\ell(e))$ is a factor of $F_{G}$.

Example 4.10. Using the notation from Example 4.3, we have that both $1-x$ and $1-y$ are factors of $F_{G}$. Factoring, we have

$$
F_{G}(\ell)=(1-x)(1-y)\left(1-x-y+x y-4 x y z^{2}\right) .
$$

Another case where there is a factorization of $F_{G}$ is when the edge involution $e \leftrightarrow \bar{e}$ is a graph automorphism of $G$. There are only two types of graphs for which such an automorphism exists:

(1) the $r$-rose, $\mathcal{R}_{r}$;

(2) the graph $\Theta_{r}$ with vertices, $v$ and $w$, and edges $e_{1}, \ldots, e_{r+1}$ where $o\left(e_{i}\right)=v$ and $\tau\left(e_{i}\right)=w$ for $i=1, \ldots, r+1$.

In this case ordering the edges in $E_{+}$first and then ordering the edges in $E-E_{+}$ accordingly, we have that

$$
A_{G}=\left[\begin{array}{cc}
B_{G} & B_{G}^{\prime} \\
B_{G}^{\prime} & B_{G}
\end{array}\right]
$$

for two matrices $B_{G}, B_{G}^{\prime} \in \operatorname{Mat}_{\left|E_{+}\right|}(\mathbb{R})$. Thus

$$
\begin{aligned}
F_{G}(\ell) & =\operatorname{det}\left(I-A_{G, \ell}\right)=\operatorname{det}\left[\begin{array}{cc}
I-B_{G, \ell} & -B_{G, \ell}^{\prime} \\
-B_{G, \ell}^{\prime} & I-B_{G, \ell}
\end{array}\right] \\
& =\operatorname{det}\left(I-B_{G, \ell}-B_{G, \ell}^{\prime}\right) \operatorname{det}\left(I-B_{G, \ell}+B_{G, \ell}^{\prime}\right) .
\end{aligned}
$$

Since each row of $B_{G}$ or $B_{G}^{\prime}$ corresponds to an edge $e \in E_{+}$the notation $B_{G, \ell}$ and $B_{G, \ell}^{\prime}$ still makes sense. 
For $\mathcal{R}_{r}$, we have that $B_{\mathcal{R}_{r}}$ is the $r \times r$ matrix consisting of all $1 \mathrm{~s}$ and $B_{\mathcal{R}_{r}}^{\prime}=B_{\mathcal{R}_{r}}-I$. In this case

$$
\operatorname{det}\left(I-B_{\mathcal{R}_{r}, \ell}+B_{\mathcal{R}_{r}, \ell}^{\prime}\right)=\prod_{e \in E_{+}}(1-\exp (-\ell(e)))
$$

These are precisely the factors which were observed above for loop edges.

For $\Theta_{r}$, we have that $B_{\Theta_{r}}$ is the $(r+1) \times(r+1)$ matrix consisting of all $0 \mathrm{~s}$ and $B_{\Theta_{r}}^{\prime}$ is the $(r+1) \times(r+1)$ matrix where all diagonal entries are 0 and all non-diagonal entries are 1 . In this case we have

$$
F_{\Theta_{r}}(\ell)=\operatorname{det}\left(I-B_{\Theta_{r}, \ell}^{\prime}\right) \operatorname{det}\left(I+B_{\Theta_{r}, \ell}^{\prime}\right) .
$$

In general, we now construct a graph quotient $D_{G} \rightarrow \bar{D}_{G}$ that identifies certain edge pairs $\{e, \bar{e}\}$ resulting in a new matrix $\bar{A}_{G}$, which selects the appropriate factor. In this new matrix, every row corresponds to either an edge $e \in E$ or an edge pair $\{e, \bar{e}\}$, and so we can still make sense of $\bar{A}_{G, f}$ for a function $f: E_{+} \rightarrow \mathbb{R}$.

When $G$ is $\mathcal{R}_{r}$ or $\Theta_{r}$, we take $\bar{D}_{\Theta_{r}}$ to be the quotient of $D_{\Theta_{r}}$ by the orientation-reversing automorphism $e \mapsto \bar{e}$. In this case $\bar{A}_{G, \ell}=B_{G, \ell}+B_{G, \ell}^{\prime}$.

Otherwise, for each pair $\{e, \bar{e}\}$ that is a loop edge of $G$, we identify the vertices of $D_{G}$ corresponding to $e$ and $\bar{e}$, now denoted $e \bar{e}$, keep the incoming edges and identify the outgoing edges that have the same terminal vertex. We call the resulting graph $\bar{D}_{G}$. Algebraically, we add together the columns corresponding to $e$ and $\bar{e}$ and delete one of the rows corresponding to $e$ and $\bar{e}$.

We define $\bar{F}_{G}: \mathcal{M}(G) \rightarrow \mathbb{R}$ by

$$
\bar{F}_{G}(\ell)=\operatorname{det}\left(I-\bar{A}_{G, \ell}\right) .
$$

The formula in Theorem 4.2, the formulas for the partial derivatives in (4.2) and (4.3), and the inner products in Lemmas 4.7 and 4.8 hold for $\bar{F}_{G}$ using the complex $\bar{C}_{G}$, which is the cycle complex of the directed graph $\bar{D}_{G}$.

Example 4.11. For $G$ equal to the barbell graph as in Example 4.1 we have $\bar{A}_{G}$ and $\bar{D}_{G}$ as shown below (columns of the matrix are ordered as $a \bar{a}, b \bar{b}, c, \bar{c}$ ):

$$
\left[\begin{array}{llll}
1 & 0 & 1 & 0 \\
0 & 1 & 0 & 1 \\
0 & 2 & 0 & 0 \\
2 & 0 & 0 & 0
\end{array}\right]
$$

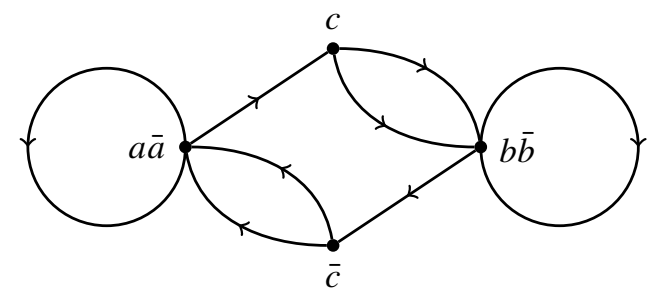

The two directed edges from $\bar{c}$ to $a \bar{a}$ are identified with the set $\{a, \bar{a}\}$ so that we think of the sequence $c, a$ or $c, \bar{a}$ as specifying which of the two edges between $\bar{c}$ and $a \bar{a}$ to traverse in a cycle. There are six simple cycles: $\gamma_{a \bar{a}}=(a \bar{a}), \gamma_{b \bar{b}}=(b \bar{b}), \gamma_{a b}=(a, c, b, \bar{c})$, $\gamma_{a \bar{b}}=(a, c, \bar{b}, \bar{c}), \gamma_{\bar{a} b}=(\bar{a}, c, b, \bar{c})$ and $\gamma_{\bar{a} \bar{b}}=(\bar{a}, c, \bar{b}, \bar{c})$. The cycle complex $\bar{C}_{G}$ is 
shown below:

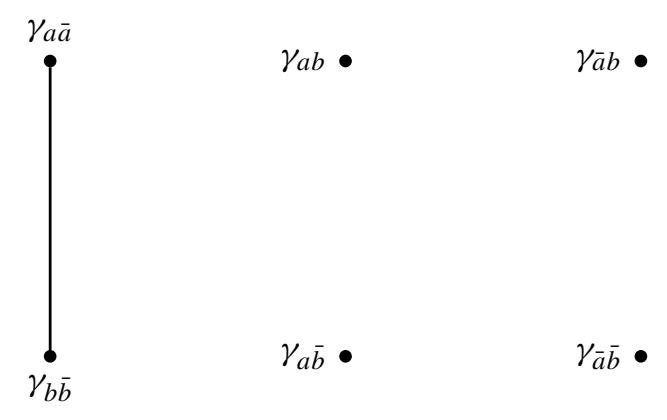

Using Theorem 4.2, we find (with $x=\exp (-\ell(a)), y=\exp (-\ell(b)$ ) and $z=$ $\exp (-\ell(c)))$ that

$$
\bar{F}_{G}(\ell)=\sum_{\Delta \in \bar{C}_{G}}(-1)^{|\Delta|} \exp (-\ell(\Delta))=1-\left(x+y+4 x y z^{2}\right)+x y .
$$

The astute reader will notice the comparison with Example 4.10.

LEMMA 4.12. With the above setup, $\operatorname{spec}\left(\bar{A}_{G, \ell}\right)=\operatorname{spec}\left(A_{G, \ell}\right)$.

Proof. Each circuit in $\bar{D}_{G}$ lifts to at most two circuits of the same length in $D_{G}$. Thus $\operatorname{tr}\left(\bar{A}_{G, \ell}^{n}\right) \leq \operatorname{tr}\left(A_{G, \ell}^{n}\right) \leq 2 \operatorname{tr}\left(\bar{A}_{G, \ell}^{n}\right)$ for all $n \in \mathbb{N}$ and so the lemma follows.

In particular, we have that $\mathfrak{P}_{G}(-\ell)=\log \operatorname{spec}\left(\bar{A}_{G, \ell}\right)$. As in Corollary 4.5 , we have the following statement. This follows for the same reasons as in Lemma 4.4 as $\bar{F}_{G}(\ell)=0$ and $\nabla \bar{F}_{G}(\ell) \neq 0$ for $\ell \in \mathcal{M}^{1}(G)$.

PROPOSITION 4.13. The unit-entropy moduli space $\mathcal{M}^{1}(G)$ is a connected component of the level set $\left\{\ell \in \mathcal{M}(G) \mid \bar{F}_{G}(\ell)=0\right\}$.

We also observe that the formulas for the metrics in Proposition 4.6 hold for $\bar{F}_{G}$.

Proposition 4.14. If $\ell_{t}:(-1,1) \rightarrow \mathcal{M}^{1}(G)$ is a smooth path, then

$$
\begin{aligned}
&\left\|\left(\ell_{t}, \dot{\ell}_{t}\right)\right\|_{\mathfrak{h}, G}^{2}=\frac{-\left\langle\dot{\ell}_{t}, \mathbf{H}\left[\bar{F}_{G}\left(\ell_{t}\right)\right] \dot{\ell}_{t}\right\rangle}{\left\langle\ell_{t}, \nabla \bar{F}_{G}\left(\ell_{t}\right)\right\rangle}=\frac{\left\langle\ddot{\ell}_{t}, \nabla \bar{F}_{G}\left(\ell_{t}\right)\right\rangle}{\left\langle\ell_{t}, \nabla \bar{F}_{G}\left(\ell_{t}\right)\right\rangle}, \\
&\left\|\left(\ell_{t}, \dot{\ell}_{t}\right)\right\|_{\mathfrak{P}, G}^{2}=\frac{-\left\langle\dot{\ell}_{t}, \mathbf{H}\left[\bar{F}_{G}\left(\ell_{t}\right)\right] \dot{\ell}_{t}\right\rangle}{\left\|\nabla \bar{F}_{G}\left(\ell_{t}\right)\right\|_{1}}=\frac{\left\langle\ddot{\ell}_{t}, \nabla \bar{F}_{G}\left(\ell_{t}\right)\right\rangle}{\left\|\nabla \bar{F}_{G}\left(\ell_{t}\right)\right\|_{1}} .
\end{aligned}
$$

5. The topology induced by the entropy metric

The purpose of this section is to show that the metric topology induced by $d_{\mathfrak{h}}$ on $\mathcal{X}^{1}\left(\mathbb{F}_{r}\right)$ is the same as the weak topology on $\mathcal{X}^{1}\left(\mathbb{F}_{r}\right)$. We do so using the formulas for the entropy metric derived in $\S 4$ and seeing that they behave as one might anticipate with regards to collapses. We refer the reader back to $\$ 2.2$ for the notation used in this section.

By Theorem 3.5 we have that $\mathfrak{h}_{G}: \mathcal{M}(G) \rightarrow \mathbb{R}$ extends to a continuous function on $\overline{\mathcal{M}}(G)$. Indeed, if $c: G \rightarrow G_{0}$ is a collapse, then for $\ell \in \mathcal{M}\left(G_{0}\right)$ we have $\mathfrak{h}_{G}\left(c^{*}(\ell)\right)=$ 
$\mathfrak{h}_{G_{0}}(\ell)$. We set $\overline{\mathcal{M}}^{1}(G)=\left\{\ell \in \overline{\mathcal{M}}(G) \mid \mathfrak{h}_{G}(\ell)=1\right\}$ and observe that we have

$$
\overline{\mathcal{M}}^{1}(G)=\bigcup_{c: G \rightarrow G_{0}} c^{*}\left(\mathcal{M}^{1}\left(G_{0}\right)\right)
$$

as well. This set is homeomorphic to the closure of $C V(G, \rho)$ in $C V\left(\mathbb{F}_{r}\right)$ for any marking $\rho: \mathcal{R}_{r} \rightarrow G$. Given a graph $G$, we observe that $F_{G}: \mathcal{M}(G) \rightarrow \mathbb{R}$ admits an extension (still denoted $F_{G}$ ) to $\mathbb{R}^{\left|E_{+}\right|}$. In particular, $F_{G}$ is defined on $\overline{\mathcal{M}}(G) \subset \mathbb{R}^{\left|E_{+}\right|}$. The next result shows that this function behaves as expected with respect to collapses.

LEMMA 5.1. If $c: G \rightarrow G_{0}$ is a collapse, then $F_{G} \circ c^{*}=F_{G_{0}}$.

Proof. It suffices to consider the case when $c: G \rightarrow G_{0}$ is the collapse of a single edge $e \in E_{+}$. Order the edges in $E$ starting with $e$ and $\bar{e}$. Since $e$ can be collapsed, it is not a loop and so we have that $A_{G}(e, e)=A_{G}(\bar{e}, \bar{e})=0$. By definition, $A_{G}(e, \bar{e})=A_{G}(\bar{e}, e)=0$. Thus the top-leftmost $2 \times 2$ block of the matrix $I-A_{G}$ is the $2 \times 2$ identity matrix.

Let $\ell \in \mathcal{M}\left(G_{0}\right)$. For an edge $e^{\prime} \notin\{e, \bar{e}\}$, the entry $\left[I-A_{G, c^{*}(\ell)}\right]\left(e, e^{\prime}\right)$ is either -1 or 0 depending on whether or not $e^{\prime}$ can follow $e$. Likewise for $\left[I-A_{G, c^{*}(\ell)}\right]\left(\bar{e}, e^{\prime}\right)$. Again, as $e$ is not a loop, for any edge $e^{\prime} \notin\{e, \bar{e}\}$, at most one of these entries is non-zero.

For each edge $e^{\prime} \notin\{e, \bar{e}\}$, where $\left[I-A_{G, c^{*}(\ell)}\right]\left(e, e^{\prime}\right)=-1$, we consider the column operation that adds the column for $e$ to the column for $e^{\prime}$. This zeros the $\left(e, e^{\prime}\right)$ entry. The $\left(\bar{e}, e^{\prime}\right)$ entry was previously 0 and is unaffected by this operation. We next see what effect this has on the remaining rows. In the row for $e^{\prime \prime} \notin\{e, \bar{e}\}$, this adds $-\exp \left(-\ell\left(e^{\prime \prime}\right)\right)$ to $A_{G, c^{*}(\ell)}\left(e^{\prime \prime}, e^{\prime}\right)$ if $e$ can follow $e^{\prime \prime}$ and 0 otherwise. In the former case, the previous entry was either $0\left(e^{\prime} \neq e^{\prime \prime}\right)$ or $1\left(e^{\prime}=e^{\prime \prime}\right)$ as $e$ is not a loop edge. In other words, this adds $-\exp \left(-\ell\left(e^{\prime \prime}\right)\right)$ whenever $e^{\prime}$ can follow $e^{\prime \prime}$ in $G_{0}$. Therefore, the remaining entries in the column for $e^{\prime}$ agree with the corresponding entries in the column of $I-A_{G_{0}, \ell}$ for $e^{\prime}$.

Hence, after performing column operations to $I-A_{G, c^{*}(\ell)}$ with the column for $e$ to clear out the rest of the row for $e$ and column operations with the column for $\bar{e}$ to clear out the rest of the row for $\bar{e}$, the resulting matrix has lower block triangular form. The top-leftmost $2 \times 2$ block is still the $2 \times 2$ identity matrix and the bottom-rightmost $(|E|-2) \times(|E|-2)$ block is $I-A_{G_{0}, \ell}$.

As these column operations do not change the determinant, we have for $\ell \in \mathcal{M}\left(G_{0}\right)$ that

$$
F_{G}\left(c^{*}(\ell)\right)=\operatorname{det}\left(I-A_{G, c^{*}(\ell)}\right)=\operatorname{det}\left(I-A_{G_{0}, \ell}\right)=F_{G_{0}}(\ell) .
$$

As a consequence of Lemma 5.1 we deduce the following result. If $c: G \rightarrow G_{0}$ is a collapse and $e \in E_{+}$is an edge such that $c(e)$ is not a vertex, then $\partial_{c(e)} F_{G_{0}}(\ell)=$ $\partial_{e} F_{G}\left(c^{*}(\ell)\right)$ for all $\ell \in \mathcal{M}\left(G_{0}\right)$. Similarly, in this same setting, if additionally $c\left(e^{\prime}\right)$ is not a vertex for an edge $e^{\prime} \in E_{+}$, then $\partial_{c(e) c\left(e^{\prime}\right)} F_{G_{0}}(\ell)=\partial_{e e^{\prime}} F_{G}\left(c^{*}(\ell)\right)$.

The tangent bundle $T \overline{\mathcal{M}}^{1}(G)$ is the subspace of $(\ell, \mathbf{v}) \in \mathbb{R}^{\left|E_{+}\right|} \times \mathbb{R}^{\left|E_{+}\right|}$such that $\ell \in \overline{\mathcal{M}}^{1}(G)$ and $\left\langle\mathbf{v}, \nabla F_{G}(\ell)\right\rangle=0$. Given a collapse, we let $\mathbf{c}^{*}: \mathbb{R}^{\left|\left(E_{0}\right)_{+}\right|} \rightarrow \mathbb{R}^{\left|E_{+}\right|}$be the derivative of the map $c^{*}: \mathcal{M}\left(G_{0}\right) \rightarrow \mathcal{M}(G)$ and $T c^{*}: T \mathcal{M}^{1}\left(G_{0}\right) \rightarrow T \overline{\mathcal{M}}^{1}(G)$ be the map given by $T c^{*}(\ell, \mathbf{v})=\left(c^{*}(\ell), \mathbf{c}^{*}(\mathbf{v})\right)$. 
Using this notation, we see that

$$
\begin{aligned}
\left\langle\mathbf{c}^{*}(\mathbf{v}), \mathbf{H}\left[F_{G}\left(c^{*}(\ell)\right)\right] \mathbf{c}^{*}(\mathbf{v})\right\rangle & =\left\langle\mathbf{v}, \mathbf{H}\left[F_{G_{0}}(\ell)\right] \mathbf{v}\right\rangle, \\
\left\langle c^{*}(\ell), \nabla F_{G}\left(c^{*}(\ell)\right)\right\rangle & =\left\langle\ell, \nabla F_{G_{0}}(\ell)\right\rangle .
\end{aligned}
$$

Hence, by Proposition 4.6, we get the following proposition.

Proposition 5.2. Let $G$ be a finite connected graph. The entropy norm $\|\cdot\|_{\mathfrak{h}, G}$ : $T \mathcal{M}^{1}(G) \rightarrow \mathbb{R}$ extends to a continuous semi-norm $\|\cdot\|_{\mathfrak{h}, G}: T \overline{\mathcal{M}}^{1}(G) \rightarrow \mathbb{R}$. Specifically, if $c: G \rightarrow G_{0}$ is a collapse and $(\ell, \mathbf{v})=T c^{*}\left(\ell_{0}, \mathbf{v}_{0}\right)$, then the extension satisfies

$$
\|(\ell, \mathbf{v})\|_{\mathfrak{h}, G}=\left\|\left(\ell_{0}, \mathbf{v}_{0}\right)\right\|_{\mathfrak{h}, G_{0}} .
$$

Consequently, we see that the distance function $d_{\mathfrak{h}, G}$ extends to a distance function on $\overline{\mathcal{M}}^{1}(G)$ and that the induced topology is the same as the subspace topology. As $X^{1}\left(\mathbb{F}_{r}\right)$ is locally finite, the metric topology agrees with the weak topology, as we now show.

THEOREM 5.3. The metric topology on $\left(\mathcal{X}^{1}\left(\mathbb{F}_{r}\right), d_{\mathfrak{h}}\right)$ is the same as the weak topology on $X^{1}\left(\mathbb{F}_{r}\right)$.

Proof. Let $U \subseteq \mathcal{X}^{1}\left(\mathbb{F}_{r}\right)$ be an open set in the weak topology and fix a marked metric graph $x=[(G, \rho, \ell)] \in U$. There are finitely many marked graphs $\left(G_{1}, \rho_{1}\right), \ldots,\left(G_{n}, \rho_{n}\right)$ such that $(G, \rho) \leq\left(G_{i}, \rho_{i}\right)$. By definition of the weak topology, the set $U \cap \overline{\mathcal{X}}^{1}\left(G_{i}, \rho_{i}\right)$ is open in $\bar{X}^{1}\left(G_{i}, \rho_{i}\right)$ in the subspace topology inherited from $\mathbb{R}_{\geq 0}^{\left|\left(E_{i}\right)_{+}\right|}$, where $E_{i}$ is the set of edges for $G_{i}$. As remarked above after Proposition 5.2, this set is also open in the metric topology induced by $d_{\mathfrak{h}, G_{i}}$. Hence there is an $\epsilon_{i}>0$ such that

$$
\left\{y \in \overline{\mathcal{X}}^{1}\left(G_{i}, \rho_{i}\right) \mid d_{\mathfrak{h}, G_{i}}(x, y)<\epsilon_{i}\right\} \subseteq U \cap \overline{\mathcal{X}}^{1}\left(G_{i}, \rho_{i}\right) .
$$

Let $\epsilon=\min \left\{\epsilon_{i} \mid 1 \leq i \leq n\right\}$. As $d_{\mathfrak{h}}(x, y) \leq d_{\mathfrak{h}, G_{i}}(x, y)$ for any $y \in \overline{\mathcal{X}}^{1}\left(G_{i}, \rho_{i}\right)$ we have

$$
\left\{y \in \overline{\mathcal{X}}^{1}\left(\mathbb{F}_{r}\right) \mid d_{\mathfrak{h}}(x, y)<\epsilon\right\} \subseteq \bigcup_{i=1}^{n} U \cap \overline{\mathcal{X}}^{1}\left(G_{i}, \rho_{i}\right) \subseteq U .
$$

Hence the metric topology is finer than the subspace topology.

Next, fix a marked metric graph $x=[(G, \rho, \ell)] \in \mathcal{X}^{1}\left(\mathbb{F}_{r}\right)$ and let $\epsilon>0$. Enumerate the finitely many marked graphs $\left(G_{1}, \rho_{1}\right), \ldots,\left(G_{n}, \rho_{n}\right)$ such that $(G, \rho) \leq\left(G_{i}, \rho_{i}\right)$ and such that $G_{i}$ is trivalent. In other words, $\left(G_{i}, \rho_{i}\right)$ are maximal elements in the partial order on marked graphs. As the norm varies continuously by Proposition 5.2, there is an $L$ and an open neighborhood $V \subseteq \bigcup_{i=1}^{n} \bar{X}^{1}\left(G_{i}, \rho_{i}\right)$ of $x$ in the weak topology such that $\|(y, \mathbf{v})\|_{\mathfrak{h}, G_{i}} \leq L$ whenever $y \in \overline{\mathcal{X}}^{1}\left(G_{i}\right) \cap V$ and $\langle\mathbf{v}, \mathbf{v}\rangle=1$. Therefore, there is an open neighborhood $U$ of $x$ in the weak topology such that

$$
U \subseteq\left\{y \in \overline{\mathcal{X}}^{1}\left(\mathbb{F}_{r}\right) \mid d_{\mathfrak{h}}(x, y)<\epsilon\right\} .
$$

Hence the subspace topology is finer than the metric topology. 
6. The entropy metric on $\mathcal{X}^{1}\left(\mathbb{F}_{2}\right)$

The goal of this section is to show that $\left(\mathcal{X}^{1}\left(\mathbb{F}_{2}\right), d_{\mathfrak{h}}\right)$ is complete. This appears as Proposition 6.8 in $\S 6.4$. The results in this section are not necessary for the remainder of the paper and can safely be skipped by a reader primarily interested in Theorems 1.2 and 1.3. However, the calculations can serve as a good introduction to estimating lengths with the entropy metric. In particular, the main strategy in each of Lemmas 6.1, 6.3 and 6.5 is very similar to the main strategy of Lemma 7.10 in $\$ 7.3$ which is the key tool used to show that $\left(\mathcal{M}^{1}\left(\mathcal{R}_{r}\right), d_{\mathfrak{h}, \mathcal{R}_{r}}\right)$ has infinite diameter.

To begin, we analyze the metric for each of the three topological types of graphs that appear in rank 2: the 2-rose $\mathcal{R}_{2}$, the barbell graph $\mathcal{B}_{2}$ and the theta graph $\Theta_{2}$. We refer the reader back to Figure 2 for these graphs. To this end, we define a continuous function $\mathrm{m}: \mathcal{X}^{1}\left(\mathbb{F}_{2}\right) \rightarrow \mathbb{R}$ which is a slight variation of the volume function in that it counts separating edges twice. In particular, it does not depend on the marking. The exact definition appears in $\S 6.4$. The strategy is to show that for any path $\ell_{t}:[0,1] \rightarrow \mathcal{M}^{1}(G)$ for $G \in\left\{\mathcal{R}_{2}, \mathcal{B}_{2}, \Theta_{2}\right\}$, if $\mathrm{m}\left(\ell_{0}\right)$ and $\mathrm{m}\left(\ell_{1}\right)$ are large enough, then the length of $\ell_{t}$ is bounded below by

$$
\frac{1}{\sqrt{5}}\left(\sqrt{\mathrm{m}\left(\ell_{1}\right)}-\sqrt{\mathrm{m}\left(\ell_{0}\right)}\right) .
$$

These calculations appear in the next three sections (Propositions 6.2, 6.4, and 6.6).

Using these estimates, it is not too hard to see that if $\left(x_{n}\right)_{n \in \mathbb{N}} \subset \mathcal{X}^{1}\left(\mathbb{F}_{2}\right)$ is Cauchy, then there is an $L$ such that $\mathrm{m}\left(x_{n}\right) \leq L$ for all $n$ (Lemma 6.7). From here, using local finiteness of $\mathcal{X}^{1}\left(\mathbb{F}_{2}\right)$ and a compactness argument, the completeness of $\left(\mathcal{X}^{1}\left(\mathbb{F}_{2}\right), d_{\mathfrak{h}}\right)$ follows. In the calculations, we make use of Lemmas 4.7 and 4.8 .

6.1. The 2-rose. Denote the edges of $\mathcal{R}_{2}$ by $e_{1}$ and $e_{2}$. To make the calculations in this subsection easier to read, given a length function $\ell \in \mathcal{M}\left(\mathcal{R}_{2}\right)$, we set $a=\ell\left(e_{1}\right), b=\ell\left(e_{2}\right)$ and $m=\ell\left(e_{1}\right)+\ell\left(e_{2}\right)$. Applying the definition of $\bar{F}_{G}$ from $\S 4.2$ to $\mathcal{R}_{2}$, we find the formula

$$
\bar{F}_{\mathcal{R}_{2}}(\ell)=1-\exp (-a)-\exp (-b)-3 \exp (-m) .
$$

LEMMA 6.1. Suppose $\ell_{t}:[0,1] \rightarrow \mathcal{M}^{1}\left(\mathcal{R}_{2}\right)$ is a smooth path such that for all $t \in[0,1]$ we have $\dot{m}_{t}>0$. If $m_{0} \geq 4$, then

$$
\mathcal{L}_{\mathfrak{h}, \mathcal{R}_{2}}\left(\ell_{t} \mid[0,1]\right) \geq \sqrt{m_{1}}-\sqrt{m_{0}} .
$$

Proof. Suppose that $\ell_{t}:[0,1] \rightarrow \mathcal{M}^{1}\left(\mathcal{R}_{2}\right)$ is a path where $\dot{m}_{t}>0$ as in the statement of the lemma and assume that $m_{0} \geq 4$. We reparametrize the path $\ell_{t}$ so that $m_{t}=t$.

Let $n_{t}=\min \left\{a_{t}, b_{t}\right\}$. As $\bar{F}_{\mathcal{R}_{2}}\left(\ell_{t}\right)=0$, we have

$$
1-2 \exp \left(-n_{t}\right) \leq 1-\exp \left(-a_{t}\right)-\exp \left(-b_{t}\right)=3 \exp \left(-m_{t}\right)=3 \exp (-t) .
$$

In particular, $2 \exp \left(-n_{t}\right) \geq 1-3 \exp (-t) \geq 2 \exp (-1)$ as $t \geq 4$ and so $n_{t}<1$ for all $t$.

Setting $p_{t}=\max \left\{a_{t}, b_{t}\right\}$, we find that $p_{t}=t-n_{t} \geq t-1$. Therefore, as $t-1 \geq 1$ for $t \geq 4$, the edge that realizes the minimum of $\left\{a_{t}, b_{t}\right\}$ does not depend on $t$ and hence 
we may assume that $b_{t}=n_{t}$ and that $a_{t}=p_{t} \geq t-1$. This gives us that $\exp \left(-a_{t}\right) \leq$ $\exp (-t+1)$. Hence, again as $\bar{F}_{\mathcal{R}_{2}}\left(\ell_{t}\right)=0$, we have

$$
1-\exp \left(-b_{t}\right)=\exp \left(-a_{t}\right)+3 \exp \left(-m_{t}\right) \leq(\exp (1)+3) \exp (-t) \leq 8 \exp (-t) .
$$

This enables us to give an upper bound on the denominator in the expression for the entropy norm. Specifically, using the fact that $x \exp (-x) \leq 1-\exp (-x)$ for $x \geq 0$, we have

$$
\begin{aligned}
\left\langle\ell_{t}, \nabla \bar{F}_{\mathcal{R}_{2}}\left(\ell_{t}\right)\right\rangle & =a_{t} \exp \left(-a_{t}\right)+b_{t} \exp \left(-b_{t}\right)+3 m_{t} \exp \left(-m_{t}\right) \\
& \leq t \exp (-t+1)+\left(1-\exp \left(-b_{t}\right)\right)+3 t \exp (-t) \\
& \leq 8 t \exp (-t)+8 \exp (-t) \\
& \leq 12 t \exp (-t) .
\end{aligned}
$$

In the final inequality we used that fact that $t \geq 4$. The expression for $\left\langle\ell_{t}, \nabla \bar{F}_{\mathcal{R}_{2}}\left(\ell_{t}\right)\right\rangle$ can be computed either directly from (6.1) or via Lemma 4.7.

Next, we get an upper bound on the numerator in the expression for the entropy norm by just using $m_{t}$. Specifically,

$$
\begin{aligned}
-\left\langle\dot{\ell}_{t}, \mathbf{H}\left[\bar{F}_{\mathcal{R}_{2}}\left(\ell_{t}\right)\right] \dot{\ell}_{t}\right\rangle & =\left(\dot{a}_{t}\right)^{2} \exp \left(-a_{t}\right)+\left(\dot{b}_{t}\right)^{2} \exp \left(-b_{t}\right)+3\left(\dot{m}_{t}\right)^{2} \exp \left(-m_{t}\right) \\
& \geq 3 \exp (-t) .
\end{aligned}
$$

As above, the expression for $-\left\langle\dot{\ell}_{t}, \mathbf{H}\left[\bar{F}_{\mathcal{R}_{2}}\left(\ell_{t}\right)\right] \dot{\ell}_{t}\right\rangle$ can be computed either directly from (6.1) or via Lemma 4.8.

Hence we find that the entropy norm along this path is bounded below by

$$
\left\|\left(\ell_{t}, \dot{\ell}_{t}\right)\right\|_{\mathfrak{h}, \mathcal{R}_{2}}^{2}=\frac{-\left\langle\dot{\ell}_{t}, \mathbf{H}\left[\bar{F}_{\mathcal{R}_{2}}\left(\ell_{t}\right)\right] \dot{\ell}_{t}\right\rangle}{\left\langle\ell_{t}, \nabla \bar{F}_{\mathcal{R}_{2}}\left(\ell_{t}\right)\right\rangle} \geq \frac{1}{4 t} .
$$

Therefore the length of this path in the entropy metric is at least

$$
\int_{m_{0}}^{m_{1}} \sqrt{\frac{1}{4 t}} d t=\sqrt{m_{1}}-\sqrt{m_{0}} .
$$

Using this lemma, we can get a lower bound on the distance between length functions in $\mathcal{M}^{1}\left(\mathcal{R}_{2}\right)$ in terms of the sum of the lengths of edges, so long as they are sufficiently large.

Proposition 6.2. Suppose $\ell$ and $\ell^{\prime}$ are length functions in $\mathcal{M}^{1}\left(\mathcal{R}_{2}\right)$ where $m=\ell\left(e_{1}\right)+$ $\ell\left(e_{2}\right)$ and $m^{\prime}=\ell^{\prime}\left(e_{1}\right)+\ell^{\prime}\left(e_{2}\right)$ are at least 4 . Then

$$
d_{\mathfrak{h}, \mathcal{R}_{2}}\left(\ell, \ell^{\prime}\right) \geq \sqrt{m^{\prime}}-\sqrt{m} .
$$

Proof. Let $\ell_{t}:[0,1] \rightarrow \mathcal{M}^{1}\left(\mathcal{R}_{2}\right)$ be a piecewise smooth path such that $\ell_{0}=\ell$ and $\ell_{1}=\ell^{\prime}$. Let $\delta \in[0,1]$ be the minimal value such that $m_{t} \geq 4$ for $t \in[\delta, 1]$. In particular, $m_{\delta} \leq m$.

By only considering the smooth subpaths of $\ell_{t} \mid[\delta, 1]$ for which $\dot{m}_{t}>0$, by Lemma 6.1, we find that

$$
\mathcal{L}_{\mathfrak{h}, \mathcal{R}_{2}}\left(\ell_{t} \mid[\delta, 1]\right) \geq \sqrt{m^{\prime}}-\sqrt{m_{\delta}} \geq \sqrt{m^{\prime}}-\sqrt{m}
$$


This also provides a lower bound on $\mathcal{L}_{\mathfrak{h}, \mathcal{R}_{2}}\left(\ell_{t} \mid[0,1]\right)$. Since the path was arbitrary this also is a lower bound on the distance between $\ell$ and $\ell^{\prime}$.

6.2. The barbell graph. Let $\mathcal{B}_{2}$ denote the graph with vertices $v$ and $w$, and edges $e_{1}, e_{2}$ and $e_{3}$ where $o\left(e_{1}\right)=\tau\left(e_{1}\right)=v, o\left(e_{2}\right)=\tau\left(e_{2}\right)=w$, and $o\left(e_{3}\right)=v$ and $\tau\left(e_{3}\right)=w$. To make the calculations in this section easier to read, given a length function $\ell \in \mathcal{M}\left(\mathcal{B}_{2}\right)$, we set $a=\ell\left(e_{1}\right), b=\ell\left(e_{2}\right)$ and $m=\ell\left(e_{1}\right)+\ell\left(e_{2}\right)+2 \ell\left(e_{3}\right)$. Applying the definition of $\bar{F}_{G}$ from $\S 4.2$ to $\mathcal{B}_{2}$, we find the formula

$$
\bar{F}_{\mathcal{B}_{2}}(\ell)=(1-\exp (-a))(1-\exp (-b))-4 \exp (-m) .
$$

LemMA 6.3. Suppose $\ell_{t}:[0,1] \rightarrow \mathcal{M}^{1}\left(\mathcal{B}_{2}\right)$ is a smooth path such that for all $t \in[0,1]$ we have $\dot{m}_{t}>0$. If $m_{0} \geq 4$, then

$$
\mathcal{L}_{\mathfrak{h}, \mathcal{B}_{2}}\left(\ell_{t} \mid[0,1]\right) \geq \frac{1}{\sqrt{2}}\left(\sqrt{m_{1}}-\sqrt{m_{0}}\right) .
$$

Proof. Suppose that $\ell_{t}:[0,1] \rightarrow \mathcal{M}^{1}\left(\mathcal{B}_{2}\right)$ is a path where $\dot{m}_{t}>0$ as in the statement of the lemma and assume that $m_{0} \geq 4$. We reparametrize the path $\ell_{t}$ so that $m_{t}=t$.

As $\bar{F}_{\mathcal{B}_{2}}\left(\ell_{t}\right)=0$, we have

$$
\left(1-\exp \left(-a_{t}\right)\right)\left(1-\exp \left(-b_{t}\right)\right)=4 \exp \left(-m_{t}\right)=4 \exp (-t) .
$$

This enables us to give an upper bound on the denominator in the expression for the entropy norm. Specifically, using the fact that $x \exp (-x) \leq 1-\exp (-x)$ for $x \geq 0$, we have

$$
\begin{aligned}
\left\langle\ell_{t}, \nabla \bar{F}_{\mathcal{B}_{2}}\left(\ell_{t}\right)\right\rangle= & a_{t} \exp \left(-a_{t}\right)\left(1-\exp \left(-b_{t}\right)\right) \\
& +b_{t} \exp \left(-b_{t}\right)\left(1-\exp \left(-a_{t}\right)\right)+4 m_{t} \exp \left(-m_{t}\right) \\
\leq & 2\left(1-\exp \left(-a_{t}\right)\right)\left(1-\exp \left(-b_{t}\right)\right)+4 t \exp (-t) \\
= & 4 t \exp (-t)+8 \exp (-t) \\
\leq & 8 t \exp (-t) .
\end{aligned}
$$

The penultimate line follows from (6.2), and in the final inequality we use the fact that $t \geq 4$.

Next, we get a lower bound on the numerator in the expression for the entropy norm. We claim that $-\left\langle\dot{\ell}_{t}, \mathbf{H}\left[\bar{F}_{\mathcal{B}_{2}}\left(\ell_{t}\right)\right] \dot{\ell}_{t}\right\rangle \geq\left(\dot{m}_{t}\right)^{2} \exp \left(-m_{t}\right)$. We have that

$$
\begin{aligned}
-\left\langle\dot{\ell}_{t}, \mathbf{H}\left[\bar{F}_{\mathcal{B}_{2}}\left(\ell_{t}\right)\right] \dot{\ell}_{t}\right\rangle= & \left(\dot{a}_{t}\right)^{2} \exp \left(-a_{t}\right)\left(1-\exp \left(-b_{t}\right)\right)+\left(\dot{b}_{t}\right)^{2} \exp \left(-b_{t}\right)\left(1-\exp \left(-a_{t}\right)\right) \\
& -2 \dot{a}_{t} \dot{b}_{t} \exp \left(-a_{t}-b_{t}\right)+4\left(\dot{m}_{t}\right)^{2} \exp \left(-m_{t}\right) .
\end{aligned}
$$

Therefore if $\dot{a}_{t} \dot{b}_{t}<0$ then each term is positive and so the claim holds. Therefore, we assume that $\dot{a}_{t}$ and $\dot{b}_{t}$ have the same sign. As $\left\langle\dot{\ell}_{t}, \nabla \bar{F}_{\mathcal{B}_{2}}\left(\ell_{t}\right)\right\rangle=0$, we have that

$$
4 \dot{m}_{t} \exp \left(-m_{t}\right)=-\dot{a}_{t} \exp \left(-a_{t}\right)\left(1-\exp \left(-b_{t}\right)\right)-\dot{b}_{t} \exp \left(-b_{t}\right)\left(1-\exp \left(-a_{t}\right)\right) .
$$

We write this equation as $w=u+v$. As $\bar{F}_{\mathcal{B}_{2}}\left(\ell_{t}\right)=0$, we find that

$$
\begin{aligned}
2 u v & =2 \dot{a}_{t} \dot{b}_{t} \exp \left(-a_{t}\right) \exp \left(-b_{t}\right)\left(1-\exp \left(-a_{t}\right)\right)\left(1-\exp \left(-b_{t}\right)\right) \\
& =2 \dot{a}_{t} \dot{b}_{t} \exp \left(-a_{t}-b_{t}\right)\left(4 \exp \left(-m_{t}\right)\right) .
\end{aligned}
$$


As $2 x y \leq \frac{3}{4}(x+y)^{2}$ for all $x$ and $y$, we find that

$2 \dot{a}_{t} \dot{b}_{t} \exp \left(-a_{t}-b_{t}\right)\left(4 \exp \left(-m_{t}\right)\right) \leq \frac{3}{4}\left(4 \dot{m}_{t} \exp \left(-m_{t}\right)\right)^{2}=3\left(\dot{m}_{t}\right)^{2}\left(4 \exp \left(-2 m_{t}\right)\right)$.

Therefore $2 \dot{a}_{t} \dot{b}_{t} \exp \left(-a_{t}-b_{t}\right) \leq 3\left(\dot{m}_{t}\right)^{2} \exp \left(-m_{t}\right)$. From this the claim now follows, and furthermore that

$$
-\left\langle\dot{\ell}_{t}, \mathbf{H}\left[\bar{F}_{\mathcal{B}_{2}}\left(\ell_{t}\right)\right] \dot{\ell}_{t}\right\rangle \geq\left(\dot{m}_{t}\right)^{2} \exp \left(-m_{t}\right)=\exp (-t) .
$$

Hence we find that the entropy norm along this path is bounded below by

$$
\left\|\left(\ell_{t}, \dot{\ell}_{t}\right)\right\|_{\mathfrak{h}, \mathcal{B}_{2}}^{2}=\frac{-\left\langle\dot{\ell}_{t}, \mathbf{H}\left[\bar{F}_{\mathcal{B}_{2}}\left(\ell_{t}\right)\right] \dot{\ell}_{t}\right\rangle}{\left\langle\ell_{t}, \nabla \bar{F}_{\mathcal{B}_{2}}\left(\ell_{t}\right)\right\rangle} \geq \frac{1}{8 t} .
$$

Therefore the length of this path in the entropy metric is at least

$$
\int_{m_{0}}^{m_{1}} \sqrt{\frac{1}{8 t}} d t=\frac{1}{\sqrt{2}}\left(\sqrt{m_{1}}-\sqrt{m_{0}}\right) .
$$

As for the 2-rose, we obtain the following proposition.

Proposition 6.4. Suppose $\ell$ and $\ell^{\prime}$ are length functions in $\mathcal{M}^{1}\left(\mathcal{B}_{2}\right)$ where $m=\ell\left(e_{1}\right)+$ $\ell\left(e_{2}\right)+2 \ell\left(e_{3}\right)$ and $m^{\prime}=\ell^{\prime}\left(e_{1}\right)+\ell^{\prime}\left(e_{2}\right)+2 \ell^{\prime}\left(e_{3}\right)$ are at least 4 . Then

$$
d_{\mathfrak{h}, \mathcal{B}_{2}}\left(\ell, \ell^{\prime}\right) \geq \frac{1}{\sqrt{2}}\left(\sqrt{m^{\prime}}-\sqrt{m}\right) .
$$

6.3. The theta graph. Let $\Theta_{2}$ denote the graph with vertices $v$ and $w$, and edges $e_{1}, e_{2}$ and $e_{3}$ where $o\left(e_{i}\right)=v$ and $\tau\left(e_{i}\right)=w$ for $i \in\{1,2,3\}$. To make the calculations in this section easier to read, given a length function $\ell \in \mathcal{M}\left(\Theta_{2}\right)$, we set $a=\ell\left(e_{1}\right)+\ell\left(e_{2}\right), b=$ $\ell\left(e_{2}\right)+\ell\left(e_{3}\right), c=\ell\left(e_{3}\right)+\ell\left(e_{1}\right)$ and $m=\ell\left(e_{1}\right)+\ell\left(e_{2}\right)+\ell\left(e_{3}\right)$. Applying the definition of $\bar{F}_{G}$ from $\S 4.2$ to $\Theta_{2}$, we find the formula

$$
\bar{F}_{\Theta_{2}}(\ell)=1-\exp (-a)-\exp (-b)-\exp (-c)-2 \exp (-m) .
$$

Lemma 6.5. Suppose $\ell_{t}:[0,1] \rightarrow \mathcal{M}^{1}\left(\Theta_{2}\right)$ is a smooth path such that for all $t \in[0,1]$ we have $\dot{m}_{t}>0$. If $m_{0} \geq 4$, then

$$
\mathcal{L}_{\mathfrak{h}, \Theta_{2}}\left(\ell_{t} \mid[0,1]\right) \geq \frac{1}{\sqrt{5}}\left(\sqrt{m_{1}}-\sqrt{m_{0}}\right) .
$$

Proof. Suppose that $\ell_{t}:[0,1] \rightarrow \mathcal{M}^{1}\left(\Theta_{2}\right)$ is a path where $\dot{m}_{t}>0$ as in the statement of the lemma and assume that $m_{0} \geq 4$. We reparametrize the path $\ell_{t}$ so that $m_{t}=t$.

Let $n_{t}=\min \left\{a_{t}, b_{t}, c_{t}\right\}$. As $\bar{F}_{\Theta_{2}}\left(\ell_{t}\right)=0$, we have

$$
1-3 \exp \left(-n_{t}\right) \leq 1-\exp \left(-a_{t}\right)-\exp \left(-b_{t}\right)-\exp \left(-c_{t}\right)=2 \exp \left(-m_{t}\right)=2 \exp (-t) .
$$

In particular, $3 \exp \left(-n_{t}\right) \geq 1-2 \exp (-t)>3 \exp (-2)$ as $t \geq 4$ and thus $n_{t}<2$ for all $t$.

Setting $p_{t}=\max \left\{a_{t}, b_{t}, c_{t}\right\}$ and $q_{t}=a_{t}+b_{t}+c_{t}-p_{t}-n_{t}$ so that $\left\{p_{t}, q_{t}, n_{t}\right\}=$ $\left\{a_{t}, b_{t}, c_{t}\right\}$ for all $t$, we find that $p_{t}, q_{t} \geq t-2$ as $p_{t}+q_{t}=2 t-n_{t} \geq 2 t-2$ and $p_{t}, q_{t} \leq t$. Therefore, as $t-2 \geq 2$ for $t \geq 4$, the cycle that realizes the minimum of $\left\{a_{t}, b_{t} c_{t}\right\}$ does not depend on $t$ and therefore we may assume that $c_{t}=\min \left\{a_{t}, b_{t}, c_{t}\right\}$ 
and thus $a_{t}, b_{t} \geq t-2$. Therefore $\exp \left(-a_{t}\right), \exp \left(-b_{t}\right) \leq \exp (-t+2)$. Hence, again as $\bar{F}_{\Theta_{2}}\left(\ell_{t}\right)=0$, we have

$$
\begin{aligned}
1-\exp \left(-c_{t}\right) & =\exp \left(-a_{t}\right)+\exp \left(-b_{t}\right)+2 \exp \left(-m_{t}\right) \\
& \leq(2 \exp (2)+2) \exp (-t) \leq 20 \exp (-t) .
\end{aligned}
$$

This enables us to give an upper bound on the denominator in the expression for the entropy norm. Specifically, using the fact that $x \exp (-x) \leq 1-\exp (-x)$ for $x \geq 0$, we have

$$
\begin{aligned}
\left\langle\ell_{t}, \nabla \bar{F}_{\Theta_{2}}\left(\ell_{t}\right)\right\rangle & =a_{t} \exp \left(-a_{t}\right)+b_{t} \exp \left(-b_{t}\right)+c_{t} \exp \left(-c_{t}\right)+2 m_{t} \exp \left(-m_{t}\right) \\
& \leq a_{t} \exp \left(-a_{t}\right)+b_{t} \exp \left(-b_{t}\right)+1-\exp \left(-c_{t}\right)+2 m_{t} \exp \left(-m_{t}\right) \\
& \leq t \exp (-t+2)+t \exp (-t+2)+20 \exp (-t)+2 t \exp (-t) \\
& \leq 20 t \exp (-t)+20 \exp (-t) \\
& \leq 40 t \exp (-t) .
\end{aligned}
$$

In the final inequality we used the fact that $t \geq 4$.

Next, we get a lower bound on the numerator in the expression for the entropy norm by just using $m_{t}$. Specifically,

$$
\begin{aligned}
-\left\langle\dot{\ell}_{t}, \mathbf{H}\left[\bar{F}_{\Theta_{2}}\left(\ell_{t}\right)\right] \dot{\ell}_{t}\right\rangle= & \left(\dot{a}_{t}\right)^{2} \exp \left(-a_{t}\right)+\left(\dot{b}_{t}\right)^{2} \exp \left(-b_{t}\right) \\
& +\left(\dot{c}_{t}\right)^{2} \exp \left(-c_{t}\right)+2\left(\dot{m}_{t}\right)^{2} \exp \left(-m_{t}\right) \\
\geq & 2 \exp (-t) .
\end{aligned}
$$

Hence we find that the entropy norm along this path is bounded below by

$$
\left\|\left(\ell_{t}, \dot{\ell}_{t}\right)\right\|_{\mathfrak{h}, \Theta_{2}}^{2}=\frac{-\left\langle\dot{\ell}_{t}, \mathbf{H}\left[\bar{F}_{\Theta_{2}}\left(\ell_{t}\right)\right] \dot{\ell}_{t}\right\rangle}{\left\langle\ell_{t}, \nabla \bar{F}_{\Theta_{2}}\left(\ell_{t}\right)\right\rangle} \geq \frac{1}{20 t} .
$$

Therefore the length of this path in the entropy metric is at least

$$
\int_{m_{0}}^{m_{1}} \sqrt{\frac{1}{20 t}} d t=\frac{1}{\sqrt{5}}\left(\sqrt{m_{1}}-\sqrt{m_{0}}\right) .
$$

Again, as for the 2-rose, we obtain the following proposition.

Proposition 6.6. Suppose $\ell$ and $\ell^{\prime}$ are length functions in $\mathcal{M}^{1}\left(\Theta_{2}\right)$ where $m=\ell\left(e_{1}\right)+$ $\ell\left(e_{2}\right)+\ell\left(e_{3}\right)$ and $m^{\prime}=\ell^{\prime}\left(e_{1}\right)+\ell^{\prime}\left(e_{2}\right)+\ell^{\prime}\left(e_{3}\right)$ are at least 4 . Then

$$
d_{\mathfrak{h}, \Theta_{2}}\left(\ell, \ell^{\prime}\right) \geq \frac{1}{\sqrt{5}}\left(\sqrt{m^{\prime}}-\sqrt{m}\right) .
$$

6.4. $\left(\mathcal{X}^{1}\left(\mathbb{F}_{2}\right), d_{\mathfrak{h}}\right)$ is complete. We can now prove the main result of this section that the entropy metric on $\mathcal{X}^{1}\left(\mathbb{F}_{2}\right)$ is complete. Given a marked metric graph $x=[(G, \rho, \ell)]$ in $\mathcal{X}^{1}\left(\mathbb{F}_{2}\right)$, we let

$$
\mathrm{m}(x)= \begin{cases}\ell\left(e_{1}\right)+\ell\left(e_{2}\right) & \text { if } G=\mathcal{R}_{2}, \\ \ell\left(e_{1}\right)+\ell\left(e_{2}\right)+2 \ell\left(e_{3}\right) & \text { if } G=\mathcal{B}_{2}, \\ \ell\left(e_{1}\right)+\ell\left(e_{2}\right)+\ell\left(e_{3}\right) & \text { if } G=\Theta_{2}\end{cases}
$$

We remark that $m: \mathcal{X}^{1}\left(\mathbb{F}_{2}\right) \rightarrow \mathbb{R}$ is an $\operatorname{Out}\left(\mathbb{F}_{2}\right)$-invariant continuous function. 
LEMma 6.7. Let $\left(x_{n}\right)_{n \in \mathbb{N}}$ be a Cauchy sequence in $\left(\mathcal{X}^{1}\left(\mathbb{F}_{2}\right), d_{\mathfrak{h}}\right)$. Then there is an L such that $m\left(x_{n}\right) \leq L$ for all $n$.

Proof. Suppose that $\left(x_{n}\right)_{n \in \mathbb{N}}$ is a sequence in $\mathcal{X}^{1}\left(\mathbb{F}_{2}\right)$ such that $\mathrm{m}\left(x_{n}\right) \rightarrow \infty$. We will show that $\left(x_{n}\right)_{n \in \mathbb{N}}$ is not Cauchy by showing that $\lim \sup d_{\mathfrak{h}}\left(x_{1}, x_{n}\right)=\infty$.

Let $m=\max \left\{\mathrm{m}\left(x_{1}\right), 4\right\}$. Given $N \geq 0$, we let $n$ be such that $\sqrt{\mathrm{m}\left(x_{n}\right)} \geq \sqrt{5} N+\sqrt{m}$. Consider a path $\alpha_{t}:[0,1] \rightarrow \mathcal{X}^{1}\left(\mathbb{F}_{2}\right)$ such that $\alpha_{0}=x_{1}$ and $\alpha_{1}=x_{n}$. Let $\delta \in[0,1]$ be the minimal value such that $\mathrm{m}\left(\alpha_{t}\right) \geq 4$ for all $t \in[\delta, 1]$. In particular, $\mathrm{m}\left(\alpha_{\delta}\right) \leq m$.

Combining Propositions 6.2, 6.4 and 6.6, we find that

$$
\mathcal{L}_{\mathfrak{h}}\left(\alpha_{t} \mid[\delta, 1]\right) \geq \frac{1}{\sqrt{5}}\left(\sqrt{\mathrm{m}\left(x_{n}\right)}-\sqrt{\mathrm{m}\left(\alpha_{\delta}\right)}\right) \geq \frac{1}{\sqrt{5}}\left(\sqrt{\mathrm{m}\left(x_{n}\right)}-\sqrt{m}\right) \geq N .
$$

This also provides a lower bound on $\mathcal{L}_{\mathfrak{h}}\left(\alpha_{t} \mid[0,1]\right)$. Since the path was arbitrary, this also is a lower bound on $d_{\mathfrak{h}}\left(x_{1}, x_{n}\right)$.

Therefore $d_{\mathfrak{h}}\left(x_{1}, x_{n}\right) \geq N$, showing that $\lim \sup d_{\mathfrak{h}}\left(x_{1}, x_{n}\right)=\infty$ as claimed.

Given $L \geq 0$, we set $\mathcal{X}_{L}^{1}\left(\mathbb{F}_{2}\right)=\left\{x \in \mathcal{X}^{1}\left(\mathbb{F}_{2}\right) \mid \mathrm{m}(x) \leq L\right\}$, and additionally, given a marked graph $\rho: \mathcal{R}_{2} \rightarrow G$, we set $\mathcal{X}_{L}^{1}(G, \rho)=\mathcal{X}^{1}(G, \rho) \cap \mathcal{X}_{L}^{1}\left(\mathbb{F}_{2}\right)$. We remark that the closure of $\mathcal{X}_{L}^{1}(G, \rho)$ is compact. As $\mathcal{X}^{1}\left(\mathbb{F}_{2}\right)$ is locally finite and there are only finitely many topological types of graphs, the following statement holds. For all $L, D \geq 0$, there is an $N$ such that if $x \in \mathcal{X}_{L}^{1}\left(\mathbb{F}_{2}\right)$ then there is a collection of marked graphs $\left(G_{1}, \rho_{1}\right), \ldots,\left(G_{N}, \rho_{N}\right)$ such that

$$
\left\{x^{\prime} \in \mathcal{X}_{L}^{1}\left(\mathbb{F}_{2}\right) \mid d_{\mathfrak{h}}\left(x, x^{\prime}\right) \leq D\right\} \subseteq \bigcup_{k=1}^{N} \mathcal{X}_{L}^{1}\left(G_{k}, \rho_{k}\right) .
$$

Proposition 6.8. The metric space $\left(\mathcal{X}^{1}\left(\mathbb{F}_{2}\right), d_{\mathfrak{h}}\right)$ is complete.

Proof. Let $\left(x_{n}\right)_{n \in \mathbb{N}}$ be a Cauchy sequence in $\left(\mathcal{X}^{1}\left(\mathbb{F}_{2}\right), d_{\mathfrak{h}}\right)$. By Lemma 6.7, there is an $L$ such that $\left(x_{n}\right) \subset \mathcal{X}_{L}^{1}\left(\mathbb{F}_{2}\right)$. Let $n_{0}$ be such that $d_{\mathfrak{h}}\left(x_{n}, x_{m}\right) \leq 1$ if $n, m \geq n_{0}$. By the above remark, there are finitely marked graphs $\left(G_{1}, \rho_{1}\right), \ldots,\left(G_{N}, \rho_{N}\right)$ such that

$$
\left\{x_{n} \mid n \geq n_{0}\right\} \subset\left\{x^{\prime} \in \mathcal{X}_{L}^{1}\left(\mathbb{F}_{2}\right) \mid d_{\mathfrak{h}}\left(x_{n_{0}}, x^{\prime}\right) \leq 1\right\} \subseteq \bigcup_{k=1}^{N} \mathcal{X}_{L}^{1}\left(G_{k}, \rho_{k}\right) .
$$

As the closure of this set in $\mathcal{X}^{1}\left(\mathbb{F}_{2}\right)$ is compact, the sequence $\left(x_{n}\right)_{n \in \mathbb{N}}$ converges.

\section{The moduli space of the rose}

The purpose of this section is to examine the entropy metric on the moduli space of an $r$-rose $\mathcal{M}^{1}\left(\mathcal{R}_{r}\right)$. We begin in $\$ 7.1$ by computing the function $\bar{F}_{\mathcal{R}_{r}}$ introduced in $\S 4.2$. For the $r$-rose, we can strengthen Proposition 4.13 and conclude that $\mathcal{M}^{1}\left(\mathcal{R}_{r}\right)$ equals the set of length functions $\ell$ for which $\bar{F}_{\mathcal{R}_{r}}(\ell)=0$. Next, in $\$ 7.2$ we show that $\left(\mathcal{M}^{1}\left(\mathcal{R}_{r}\right), d_{\mathfrak{h}, \mathcal{R}_{r}}\right)$ for $r \geq 3$ is not complete by producing paths that have finite length yet no accumulation point. Lastly, in $\S 7.3$ we show that $\left(\mathcal{M}^{1}\left(\mathcal{R}_{r}\right), d_{\mathfrak{h}, \mathcal{R}_{r}}\right)$ has infinite diameter. Specifically, a path that shrinks the length of an edge to zero necessarily has infinite length. 
7.1. $\mathcal{M}^{1}\left(\mathcal{R}_{r}\right)$ as a zero locus. In this section we compute the function $\bar{F}_{\mathcal{R}_{r}}$. This appears as Proposition 7.3. In Proposition 7.5 we prove that $\mathcal{M}^{1}\left(\mathcal{R}_{r}\right)=\left\{\ell \in \mathcal{M}\left(\mathcal{R}_{r}\right) \mid \bar{F}_{\mathcal{R}_{r}}(\ell)=0\right\}$; this strengthens Proposition 4.13 in this setting.

First we set some notation for working with the graph $\mathcal{R}_{r}$. We identify the unoriented edges of $\mathcal{R}_{r}$ with the set $[r]=\{1,2, \ldots, r\}$. To simplify the expressions, we will use $r$ as the identifying subscript rather than $\mathcal{R}_{r}$ and we will use variables $\ell=\left(\ell^{1}, \ldots, \ell^{r}\right)$ to denote the length of the unoriented edges. The matrix $\bar{A}_{r, \ell} \in \operatorname{Mat}_{r}(\mathbb{R})$ has rows and columns indexed by $[r]$, and we have

$$
\bar{A}_{r, \ell}(i, j)=\exp \left(-\ell^{i}\right)(2-\delta(i, j))
$$

where $\delta(\cdot, \cdot)$ is the Kronecker delta function.

For the calculations in this section, we need the following combinatorial identities.

LEMMA 7.1. For any $r \geq 1$ and any $x \in \mathbb{R}$, the following equations hold:

$$
\begin{aligned}
& (1+x)^{r-1}(x-(2 r-1))=x^{r} \sum_{k=0}^{r}\left(\begin{array}{l}
r \\
k
\end{array}\right)(1-2 k) x^{-k}, \\
& (1+x)^{r-1}(x+(2 r+1))=x^{r} \sum_{k=0}^{r}\left(\begin{array}{l}
r \\
k
\end{array}\right)(1+2 k) x^{-k} .
\end{aligned}
$$

Proof. Differentiate the equation $(1+x)^{r}=\sum_{k=0}^{r}\left(\begin{array}{l}r \\ k\end{array}\right) x^{r-k}$ and multiply it by $x$ to obtain the equality $r x(1+x)^{r-1}=\sum_{k=0}^{r}(r-k)\left(\begin{array}{l}r \\ k\end{array}\right) x^{r-k}$. Therefore

$$
\begin{aligned}
2 r x(1+x)^{r-1}-(2 r-1)(1+x)^{r}= & 2 r \sum_{k=0}^{r}\left(\begin{array}{l}
r \\
k
\end{array}\right) x^{r-k}-\sum_{k=0}^{r} 2 k\left(\begin{array}{l}
r \\
k
\end{array}\right) x^{r-k} \\
& -2 r \sum_{k=0}^{r}\left(\begin{array}{l}
r \\
k
\end{array}\right) x^{r-k}+\sum_{k=0}^{r}\left(\begin{array}{l}
r \\
k
\end{array}\right) x^{r-k} \\
= & \sum_{k=0}^{r}(1-2 k)\left(\begin{array}{l}
r \\
k
\end{array}\right) x^{r-k}=x^{r} \sum_{k=0}^{r}(1-2 k)\left(\begin{array}{l}
r \\
k
\end{array}\right) x^{-k} .
\end{aligned}
$$

The left-hand side in the above equation simplifies to $(1+x)^{r-1}(x-(2 r-1))$. This shows (7.2). In a similar manner one can derive (7.3).

COROLlaRY 7.2. For any $r \geq 1$,

$$
\sum_{k=0}^{r}\left(\begin{array}{l}
r \\
k
\end{array}\right)(1-2 k)(2 r-1)^{-k}=0 .
$$

Proof. Evaluate equation (7.2) with $x=2 r-1$. The left-hand side becomes 0. Dividing the resulting equation by $(2 r-1)^{r}$, we obtain (7.4).

Given a length function $\ell=\left(\ell^{1}, \ldots, \ell^{n}\right) \in \mathcal{M}\left(\mathcal{R}_{r}\right)$ and a subset $S \subseteq[r]$, we define $\ell^{S}=\sum_{k \in S} \ell^{k}$. In particular, we have $\ell^{\emptyset}=0$. 
PROPOSITION 7.3. For any $r \geq 2$ and any length function $\ell \in \mathcal{M}\left(\mathcal{R}_{r}\right)$,

$$
\bar{F}_{r}(\ell)=\sum_{S \subseteq[r]}(1-2|S|) \exp \left(-\ell^{S}\right) .
$$

Proof. Using the expansion of the determinant via permutations of $[r]$, we can express $\bar{F}_{r}(\ell)=\operatorname{det}\left(I-\bar{A}_{r, \ell}\right)$ as

$$
\bar{F}_{r}(\ell)=\sum_{S \subseteq[r]} c_{S, r} \exp \left(-\ell^{S}\right)
$$

for some coefficients $c_{S, r} \in \mathbb{R}$ depending on the subset $S \subseteq[r]$ and the rank $r$. Further, it is apparent that the coefficient $c_{S, r}$ only depends on the cardinality of $S$. It remains to determine these coefficients. We will do so by induction.

For $r=2$, we compute

$$
\begin{aligned}
\bar{F}_{2}\left(\ell^{1}, \ell^{2}\right) & =\operatorname{det}\left[\begin{array}{cc}
1-\exp \left(-\ell^{1}\right) & -2 \exp \left(-\ell^{1}\right) \\
-2 \exp \left(-\ell^{2}\right) & 1-\exp \left(-\ell^{2}\right)
\end{array}\right] \\
& =1-\exp \left(-\ell^{1}\right)-\exp \left(-\ell^{2}\right)-3 \exp \left(-\ell^{1}-\ell^{2}\right) .
\end{aligned}
$$

This shows the proposition for $r=2$.

Suppose $r \geq 3$ and that the proposition holds for $r-1$. That is, we assume that $c_{S, r-1}=1-2|S|$ for any $S \subseteq[r-1]$. Since $c_{S, r}$ only depends on the cardinality of $S$, this implies that $c_{S, r}=1-2|S|$ for any $S \subseteq[r]$ where $|S|<r$ as well. Hence it only remains to compute $c_{[r], r}$.

To compute $c_{[r], r}$, we make use of Corollary 7.2. Indeed, by Example 3.3, we have $\mathfrak{h}_{r}(\log (2 r-1) \cdot \mathbf{1})=1$. Therefore, by Proposition 4.13, we obtain $\bar{F}_{r}(\log (2 r-1) \cdot \mathbf{1})=0$. Hence

$$
\begin{aligned}
0 & =\bar{F}_{r}(\log (2 r-1) \cdot \mathbf{1}) \\
& =\sum_{S \subset[r]}(1-2|S|) \exp (-|S| \log (2 r-1))+c_{[r], r} \exp (-r \log (2 r-1)) \\
& =\sum_{k=0}^{r-1}\left(\begin{array}{l}
r \\
k
\end{array}\right)(1-2 k)(2 r-1)^{-k}+c_{[r], r}(2 r-1)^{-r} .
\end{aligned}
$$

By Corollary 7.2, we find that $c_{[r], r}=1-2 r$ as desired.

Example 7.4. For $r=2$ and $r=3$, using the coordinates $x=\exp \left(-\ell^{1}\right), y=\exp \left(-\ell^{2}\right)$ and $z=\exp \left(-\ell^{3}\right)$, we find

$$
\begin{aligned}
\bar{F}_{2}\left(\ell^{1}, \ell^{2}\right) & =1-x-y-3 x y, \\
\bar{F}_{3}\left(\ell^{1}, \ell^{2}, \ell^{3}\right) & =1-x-y-z-3 x y-3 x z-3 y z-5 x y z .
\end{aligned}
$$

Figure 5 shows $\mathcal{M}^{1}\left(\mathcal{R}_{r}\right)$ as a subset of $\mathcal{M}\left(\mathcal{R}_{r}\right)$ for $r=2$ and $r=3$.

Using Proposition 7.3, we can provide a strengthening of Proposition 4.13 for the $r$-rose.

PROPOSITION 7.5. For any $r \geq 2$, the unit-entropy moduli space $\mathcal{M}^{1}\left(\mathcal{R}_{r}\right)$ equals the level set $\left\{\ell \in \mathcal{M}\left(\mathcal{R}_{r}\right) \mid \bar{F}_{r}(\ell)=0\right\}$. 

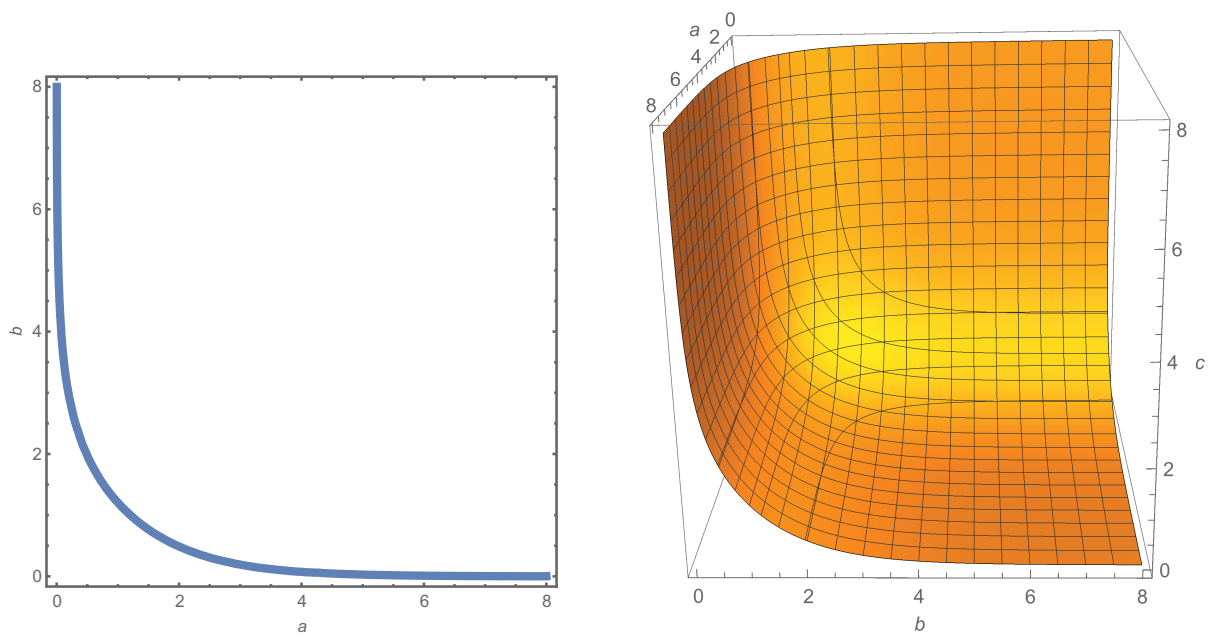

FIGURE 5. The hypersurfaces $\mathcal{M}^{1}\left(\mathcal{R}_{r}\right)$ for the roses with 2 and 3 petals.

Proof. By Proposition 4.13, we have that $\mathcal{M}^{1}\left(\mathcal{R}_{r}\right) \subseteq\left\{\ell \in \mathcal{M}\left(\mathcal{R}_{r}\right) \mid \bar{F}_{r}(\ell)=0\right\}$. Suppose that $\bar{F}_{r}(\ell)=0$ for some $\ell \in \mathcal{M}\left(\mathcal{R}_{r}\right)$. Set $h=\mathfrak{h}_{r}(\ell)$. We need to show that $h=1$.

Consider the function $p: \mathbb{R}_{>0} \rightarrow \mathbb{R}$ defined by $p(t)=\bar{F}_{r}(t \cdot \ell)$. We have $p(1)=$ $\bar{F}_{r}(\ell)=0$. As $\mathfrak{h}_{r}(h \cdot \ell)=1$, we have $p(h)=\bar{F}_{r}(h \cdot \ell)=0$ as well by Proposition 4.13.

Using the expression for $\bar{F}_{r}(\ell)$ derived in Proposition 7.3, we compute that

$$
p^{\prime}(t)=\sum_{\substack{S \subseteq[r] \\ S \neq \emptyset}}(2|S|-1) \ell^{S} \exp \left(-t \cdot \ell^{S}\right) .
$$

Therefore $p^{\prime}(t)>0$ for all $t \in \mathbb{R}_{>0}$. As $p(h \cdot \ell)=0=p(\ell)$, we must have that $h \cdot \ell=\ell$ and hence $h=1$.

7.2. Finite-length paths in $\mathcal{M}^{1}\left(\mathcal{R}_{r}\right)$ for $r \geq 3$. Using the computation of $\bar{F}_{r}$, in Proposition 7.8 we will compute the length of the path in $\mathcal{M}^{1}\left(\mathcal{R}_{r}\right)$ starting at $\log (2 r-1) \cdot 1$ that blows up the length of one edge while shrinking the lengths of the others at the same rate. As we will show, when $r$ is at least 3, this path has finite length and thus the moduli space $\left(\mathcal{M}^{1}\left(\mathcal{R}_{r}\right), d_{\mathfrak{h}, \mathcal{R}_{r}}\right)$ is not complete for $r \geq 3$.

Before we begin, it is useful to introduce the following functions $X_{i}, Y_{i}: \mathcal{M}\left(\mathcal{R}_{r}\right) \rightarrow \mathbb{R}$ for each $i \in[r]$ :

$$
\begin{aligned}
& X_{i}(\ell)=\sum_{S \subseteq[r]-\{i\}}(1-2|S|) \exp \left(-\ell^{S}\right), \\
& Y_{i}(\ell)=\sum_{S \subseteq[r]-\{i\}}(1+2|S|) \exp \left(-\ell^{S}\right) .
\end{aligned}
$$

Both $X_{i}$ and $Y_{i}$ are constant with respect to $\ell^{i}$. Using these functions, we can isolate the terms in $\bar{F}_{r}(\ell)$ in which $\ell^{i}$ appears and write

$$
\bar{F}_{r}(\ell)=X_{i}(\ell)-\exp \left(-\ell^{i}\right) Y_{i}(\ell) .
$$


Hence for $\ell \in \mathcal{M}^{1}\left(\mathcal{R}_{r}\right)$, as $\bar{F}_{r}(\ell)=0$ we can solve for $\ell^{i}$ and write

$$
\ell^{i}=\log \left(\frac{Y_{i}(\ell)}{X_{i}(\ell)}\right) .
$$

Further, we find the following expression for the partial derivative of $\bar{F}_{r}(\ell)$ with respect to $\ell^{i}$ :

$$
\partial_{i} \bar{F}_{r}(\ell)=\exp \left(-\ell^{i}\right) Y_{i}(\ell)
$$

We observe the following inequalities for any $\ell \in \mathcal{M}^{1}\left(\mathcal{R}_{r}\right)$.

LEMMA 7.6. Let $r \geq 2$ and let $\ell \in \mathcal{M}^{1}\left(\mathcal{R}_{r}\right)$. Then

$$
\begin{aligned}
& 0<X_{i}(\ell)<1, \\
& 1<Y_{i}(\ell)<4 .
\end{aligned}
$$

Proof. For (7.11), we first note that $X_{i}(\ell)=\exp \left(-\ell^{i}\right) Y_{i}(\ell)$ for all $\ell \in \mathcal{M}^{1}\left(\mathcal{R}_{r}\right)$ by Proposition 4.13 and (7.8). Since every term in $Y_{i}(\ell)$ has a positive coefficient, we find that $0<X_{i}(\ell)$. As the term in $X_{i}(\ell)$ corresponding to $S=\emptyset$ is 1 and all other terms have negative coefficients, we find $X_{i}(\ell)<1$.

For (7.12), we have that the term in $Y_{i}(\ell)$ corresponding to $S=\emptyset$ is 1 and all other terms have positive coefficients, thus $1<Y_{i}(\ell)$. The terms in $1-X_{i}(\ell)$ and $Y_{i}(\ell)-1$ correspond to the non-empty subsets $S \subseteq[r]-\{i\}$. The coefficient for the term corresponding to $S$ in $1-X_{i}(\ell)$ is

$$
\frac{2|S|+1}{2|S|-1}
$$

times the coefficient for the same term in $Y_{i}(\ell)-1$. As this ratio is bounded by 3 , we find that $Y_{i}(\ell)-1 \leq 3\left(1-X_{i}(\ell)\right)$. Hence, as $1-X_{i}(\ell)<1$ by $(7.11)$, we have $Y_{i}(\ell)-1<3$ and so $Y_{i}(\ell)<4$.

We record the following calculation.

LEMMA 7.7. Let $r \geq 2$, and let $\ell \in \mathcal{M}^{1}\left(\mathcal{R}_{r}\right)$ be such that $\ell^{i}=\log (L)$ for $i \in[r-1]$ for some $L>2 r-3$. Then

$$
\ell^{r}=\log \left(\frac{L+(2 r-1)}{L-(2 r-3)}\right) .
$$

Proof. For any $S \subseteq[r-1]$ we have $\exp \left(-\ell^{S}\right)=\exp (-|S| \log L)=L^{-|S|}$. Hence, by Lemma 7.1, we have that

$$
\begin{aligned}
& X_{r}(\ell)=\sum_{k=0}^{r-1}\left(\begin{array}{c}
r-1 \\
k
\end{array}\right)(1-2 k) L^{-k}=L^{-r+1}(L+1)^{r-2}(L-(2 r-3)), \\
& Y_{r}(\ell)=\sum_{k=0}^{r-1}\left(\begin{array}{c}
r-1 \\
k
\end{array}\right)(1+2 k) L^{-k}=L^{-r+1}(L+1)^{r-2}(L+(2 r-1)) .
\end{aligned}
$$


Therefore, by (7.9), we find that

$$
\ell^{r}=\log \left(\frac{Y_{r}(\ell)}{X_{r}(\ell)}\right)=\log \left(\frac{L+(2 r-1)}{L-(2 r-3)}\right) .
$$

For any $r \geq 3$, we will construct a path $\ell_{t}:[0,1) \rightarrow \mathcal{M}^{1}\left(\mathcal{R}_{r}\right)$ that has finite length and the property that $\ell_{t}^{r} \rightarrow \infty$ as $t \rightarrow 1^{-}$.

Proposition 7.8. Fix $r \geq 3$ and let $N_{t}=2(r-t)-1$. Let $\ell_{t}:[0,1) \rightarrow \mathcal{M}^{1}\left(\mathcal{R}_{r}\right)$ be the smooth path where $\ell_{t}^{i}=\log \left(N_{t}\right)$ for $i \in[r-1]$. Then $\mathcal{L}_{\mathfrak{h}, r}\left(\ell_{t} \mid[0,1)\right)$ is finite and $\ell_{t}^{r} \rightarrow \infty$ as $t \rightarrow 1^{-}$.

Proof. Let $\ell_{t}:[0,1) \rightarrow \mathcal{M}^{1}\left(\mathcal{R}_{r}\right)$ be as in the statement. Using Lemma 7.7, we find that

$$
\ell_{t}^{r}=\log \left(\frac{2 r-1-t}{1-t}\right)
$$

In particular, we have $\ell_{t}^{r} \rightarrow \infty$ when $t \rightarrow 1^{-}$as claimed.

We first provide a lower bound on $\left\langle\ell_{t}, \nabla \bar{F}_{r}\left(\ell_{t}\right)\right\rangle$. This is the denominator of the expression for the entropy norm in Proposition 4.14. Using the expressions for the partial derivatives for $\bar{F}_{r}\left(\ell_{t}\right)$ in (7.10), the fact that $1<Y_{i}\left(\ell_{t}\right)$ from (7.12) and that $\ell_{t}^{r} \exp \left(-\ell_{t}^{r}\right) Y_{r}\left(\ell_{t}\right)>0$, we have that

$$
\left\langle\ell_{t}, \nabla \bar{F}_{r}\left(\ell_{t}\right)\right\rangle=\sum_{i=1}^{r} \ell_{t}^{i} \exp \left(-\ell_{t}^{i}\right) Y_{i}\left(\ell_{t}\right)>\sum_{i=1}^{r-1} \ell_{t}^{i} \exp \left(-\ell_{t}^{i}\right)=(r-1) \frac{\log \left(N_{t}\right)}{N_{t}} .
$$

As $\log \left(N_{t}\right) \geq \log \left(N_{1}\right)$ and $N_{t} \leq N_{0}$ for all $t \in[0,1]$, we conclude that

$$
\left\langle\ell_{t}, \nabla \bar{F}_{r}\left(\ell_{t}\right)\right\rangle>(r-1) \frac{\log \left(N_{t}\right)}{N_{t}} \geq(r-1) \frac{\log \left(N_{1}\right)}{N_{0}}=(r-1) \frac{\log (2 r-3)}{2 r-1} .
$$

Next, we provide an upper bound on $\left\langle\ddot{\ell}_{t}, \nabla \bar{F}_{r}\left(\ell_{t}\right)\right\rangle$. This is the numerator of the expression for the entropy norm in Proposition 4.14. To do so, we compute that

$$
\dddot{\ell}_{t}^{i}=-\frac{4}{N_{t}^{2}}, \quad \text { for } i \in[r-1] \text {, and } \ddot{\ell}_{t}^{r}=-\frac{1}{(2 r-1-t)^{2}}+\frac{1}{(1-t)^{2}} .
$$

In particular, $\ddot{\ell}_{t}^{i} \exp \left(-\ell_{t}^{i}\right) Y_{i}\left(\ell_{t}\right)<0$ for $i \in[r-1]$ and $\ddot{\ell}_{t}^{r}<1 /(1-t)^{2}$. Combining these with the expressions for the partial derivatives for $\bar{F}_{r}\left(\ell_{t}\right)$ in (7.10) and the fact that $Y_{i}\left(\ell_{t}\right)<4$ (7.12), we have that

$$
\begin{aligned}
\left\langle\ddot{\ell}_{t}, \nabla \bar{F}_{r}\left(\ell_{t}\right)\right\rangle & =\sum_{i=1}^{r} \ddot{\ell}_{t}^{i} \exp \left(-\ell_{t}^{i}\right) Y_{i}\left(\ell_{t}\right) \\
& <\ddot{\ell}_{t}^{r} \exp \left(-\ell_{t}^{r}\right) Y_{i}\left(\ell_{t}\right) \\
& <\frac{1}{(1-t)^{2}} \cdot \frac{1-t}{2 r-1-t} \cdot 4 \leq \frac{2}{r-1} \cdot \frac{1}{1-t} .
\end{aligned}
$$


Proposition 4.14, together with the bounds appearing in (7.14) and (7.15), implies that

$$
\left\|\left(\ell_{t}, \dot{\ell}_{t}\right)\right\|_{\mathfrak{h}, \mathcal{R}_{r}}^{2}=\frac{\left\langle\ddot{\ell}_{t}, \nabla \bar{F}_{r}\left(\ell_{t}\right)\right\rangle}{\left\langle\ell_{t}, \nabla \bar{F}_{r}\left(\ell_{t}\right)\right\rangle} \leq \frac{2(2 r-1)}{(r-1)^{2} \log (2 r-3)} \cdot \frac{1}{1-t} .
$$

Therefore, the entropy length of the path $\ell_{t}:[0,1) \rightarrow \mathcal{M}^{1}\left(\mathcal{R}_{r}\right)$ is finite as claimed.

As a consequence, we obtain that $\mathcal{M}^{1}\left(\mathcal{R}_{r}\right)$ is not complete when $r \geq 3$.

PROPOSITION 7.9. For any $r \geq 3$, the moduli space $\left(\mathcal{M}^{1}\left(\mathcal{R}_{r}\right), d_{\mathfrak{h}, \mathcal{R}_{r}}\right)$ is not complete.

In $\S 8$ we will use Proposition 7.8 to show that $\left(\mathcal{X}^{1}\left(\mathbb{F}_{r}\right), d_{\mathfrak{h}}\right)$ is not complete as well when $r \geq 3$.

7.3. The diameter of $\mathcal{M}^{1}\left(\mathcal{R}_{r}\right)$ is infinite. In this subsection we show that $\left(\mathcal{M}^{1}\left(\mathcal{R}_{r}\right), d_{\mathfrak{h}, \mathcal{R}_{r}}\right)$ has infinite diameter by showing that any path that shrinks an edge to 0 has infinite length. Before we begin, it is useful to introduce the following functions. For distinct $i, j \in[r]$, we define

$$
\begin{aligned}
& X_{i j}(\ell)=\sum_{S \subseteq[r]-\{i, j\}}(1+2|S|) \exp \left(-\ell^{S}\right), \\
& Y_{i j}(\ell)=\sum_{S \subseteq[r]-\{i, j\}}(3+2|S|) \exp \left(-\ell^{S}\right) .
\end{aligned}
$$

As in Lemma 7.6, we observe that, for any $\ell \in \mathcal{M}^{1}\left(\mathcal{R}_{r}\right)$, we have

$$
3<Y_{i j}(\ell)<3 Y_{i}(\ell)<12 .
$$

Notice that both $X_{i j}$ and $Y_{i j}$ are constant with respect to both $\ell^{i}$ and $\ell^{j}$. For any distinct $i, j \in[r]$, these functions allow us to write

$$
Y_{i}(\ell)=X_{i j}(\ell)+\exp \left(-\ell^{j}\right) Y_{i j}(\ell) .
$$

Using (7.19) plus the expressions for the partial derivatives for $\bar{F}_{r}(\ell)$ in (7.10), we find the following expressions for the second partial derivatives of $\bar{F}_{r}(\ell)$ :

$$
\begin{gathered}
\partial_{i i} \bar{F}_{r}(\ell)=-\exp \left(-\ell^{i}\right) Y_{i}(\ell), \\
\partial_{i j} \bar{F}_{r}(\ell)=-\exp \left(-\ell^{i}-\ell^{j}\right) Y_{i j}(\ell) \text { for } i \neq j .
\end{gathered}
$$

The following technical lemma is the main tool for estimating length. Intuitively, it says that when one of the edge lengths $-\ell^{r}$ in the statement - is short, the length of a path is bounded below by the difference in the square roots of the lengths of second shortest edge $-\ell^{1}$ in the statement - at the endpoints of the path. In the statement below, shortness of $\ell^{r}$ is guaranteed by taking $\ell^{1}$ large enough.

LEMMA 7.10. Let $r \geq 2$. There is an $L_{r}$ with the following property. Suppose $\ell_{t}:[0,1] \rightarrow$ $\mathcal{M}^{1}\left(\mathcal{R}_{r}\right)$ is a piecewise smooth path such that, for all $t \in[0,1]$ :

(1) $\ell_{t}^{1}=\min \left\{\ell_{t}^{i} \mid i \in[r-1]\right\}$;

(2) $\ell_{0}^{1} \geq L_{r}$; and

(3) $\dot{\ell}_{t}^{1}>0$. 
Then

$$
\mathcal{L}_{\mathfrak{h}, \mathcal{R}_{r}}\left(\ell_{t} \mid[0,1]\right) \geq \frac{1}{2 \sqrt{2} B_{1}}\left(\sqrt{B_{1} \ell_{1}^{1}+B_{2}}-\sqrt{B_{1} \ell_{0}^{1}+B_{2}}\right)
$$

where $B_{1}=4(r-1)$ and $B_{2}=2^{r+3}(2 r-1)$.

Proof. Let $\ell_{t}:[0,1] \rightarrow \mathcal{M}^{1}\left(\mathcal{R}_{r}\right)$ be as in the statement. By (3) we may reparametrize the path so that $\ell_{t}^{1}=t$. Let $L_{r}$ be large enough so that

$$
\max \left\{2^{r}(2 r-3) \exp \left(-L_{r}\right), 288 r \exp \left(-L_{r}\right)\right\} \leq 1 .
$$

The method of proof is similar to the calculations performed in $\S 6$. Specifically, using the expression

$$
\left\|\left(\ell_{t}, \dot{\ell}_{t}\right)\right\|_{\mathfrak{h}, \mathcal{R}_{r}}^{2}=\frac{-\left\langle\dot{\ell}_{t}, \mathbf{H}\left[\bar{F}_{r}\left(\ell_{t}\right)\right] \dot{\ell}_{t}\right\rangle}{\left\langle\ell_{t}, \nabla \bar{F}_{r}\left(\ell_{t}\right)\right\rangle}
$$

from Proposition 4.14, we show that the square of the entropy norm along this path is bounded from below by $1 / 2\left(B_{1} t+B_{2}\right)$. This is done by showing that the denominator is bounded from above by $\exp (-t)\left(B_{1} t+B_{2}\right)$ in $(7.27)$, and that the numerator is bounded from below by $\frac{1}{2} \exp (-t)$ in $(7.33)$.

We first provide the upper bound on $\left\langle\ell_{t}, \nabla \bar{F}_{r}\left(\ell_{t}\right)\right\rangle$.

As $\ell_{t}^{i} \geq \ell_{t}^{1}=t$ for all $i \in[r-1]$, we have that $\exp \left(-\ell_{t}^{S}\right) \leq \exp (-t)$ for all non-empty subsets $S \subseteq[r-1]$. Since $1-2|S| \geq-(2 r-3)$ for any non-empty subset $S \subseteq[r-1]$, using the definition of $X_{i}\left(\ell_{t}\right)$ in (7.6) we have that

$$
X_{r}\left(\ell_{t}\right)=\sum_{S \subseteq[r-1]}(1-2|S|) \exp \left(-\ell_{t}^{S}\right) \geq 1-2^{r-1}(2 r-3) \exp (-t) .
$$

Therefore

$$
1-X_{r}\left(\ell_{t}\right) \leq 2^{r-1}(2 r-3) \exp (-t) .
$$

As $t \geq L_{r}$ we additionally find that

$$
X_{r}\left(\ell_{t}\right) \geq \frac{1}{2}
$$

As $0<X_{r}\left(\ell_{t}\right)<1$ by (7.11) and $-\log (1-x) \leq x /(1-x)$ for all $0<x<1$, using (7.24) and (7.25), we find that

$$
-\log \left(X_{r}\left(\ell_{t}\right)\right)=-\log \left(1-\left(1-X_{r}\left(\ell_{t}\right)\right)\right) \leq \frac{1-X_{r}\left(\ell_{t}\right)}{X_{r}\left(\ell_{t}\right)} \leq 2^{r}(2 r-3) \exp (-t) .
$$

Similarly, as $1<Y_{r}\left(\ell_{t}\right)$ by (7.12) and $\log (x) \leq x-1$ for all $x \geq 1$, using the definition of $Y_{i}\left(\ell_{t}\right)$ from (7.7), we have that

$$
\log \left(Y_{r}\left(\ell_{t}\right)\right) \leq Y_{r}\left(\ell_{t}\right)-1=\sum_{\substack{S \subseteq[r-1] \\ S \neq \emptyset}}(1+2|S|) \exp \left(-\ell_{t}^{S}\right) \leq 2^{r-1}(2 r-1) \exp (-t) .
$$

Thus by (7.9), we find

$$
\ell_{t}^{r}=\log \left(Y_{r}\left(\ell_{t}\right)\right)-\log \left(X_{r}\left(\ell_{t}\right)\right) \leq 2^{r+1}(2 r-1) \exp (-t) .
$$


As $x \exp (-x)$ is decreasing for $x>1$, we have that $\ell_{t}^{i} \exp \left(-\ell_{t}^{i}\right) \leq \ell_{t}^{1} \exp \left(-\ell_{t}^{1}\right)=$ $t \exp (-t)$ for $i \in[r-1]$. Using the expressions for the partial derivatives of $\bar{F}_{r}\left(\ell_{t}\right)$ in (7.10) and the fact that $Y_{i}\left(\ell_{t}\right)<4$ from (7.12), we have that

$$
\begin{aligned}
\left\langle\ell_{t}, \nabla \bar{F}_{r}\left(\ell_{t}\right)\right\rangle & =\sum_{i=1}^{r} \ell_{t}^{i} \exp \left(-\ell_{t}^{i}\right) Y_{i}\left(\ell_{t}\right) \\
& <4\left((r-1) t \exp (-t)+\ell_{t}^{r} \exp \left(-\ell_{t}^{r}\right)\right) \\
& \leq 4 \exp (-t)\left((r-1) t+2^{r+1}(2 r-1)\right) .
\end{aligned}
$$

As defined above, we have that $B_{1}=4(r-1)$ and $B_{2}=2^{r+3}(2 r-1)$. Hence

$$
\left\langle\ell_{t}, \nabla \bar{F}_{r}\left(\ell_{t}\right)\right\rangle \leq \exp (-t)\left(B_{1} t+B_{2}\right) .
$$

Next we provide a lower bound on $-\left\langle\dot{\ell}_{t}, \mathbf{H}\left[\bar{F}_{r}\left(\ell_{t}\right)\right] \dot{\ell}_{t}\right\rangle$. Using the expressions for the second partial derivatives of $\bar{F}_{r}\left(\ell_{t}\right)$ in (7.20) and (7.21), we have that

$$
-\left\langle\dot{\ell}_{t}, \mathbf{H}\left[\bar{F}_{r}\left(\ell_{t}\right)\right] \dot{\ell}_{t}\right\rangle=\sum_{i=1}^{r}\left(\dot{\ell}_{t}^{i}\right)^{2} \exp \left(-\ell_{t}^{i}\right) Y_{i}\left(\ell_{t}\right)+\sum_{i=1}^{r-1} \sum_{j=i+1}^{r} 2 \dot{\ell}_{t}^{i} \dot{\ell}_{t}^{j} \exp \left(-\ell_{t}^{i}-\ell_{t}^{j}\right) Y_{i j}\left(\ell_{t}\right) .
$$

The following claim says that the diagonal terms in $\mathbf{H}\left[\bar{F}_{r}\left(\ell_{t}\right)\right]$ dominate in the current setting, that is, when one of the edge lengths is small.

CLAIM 7.11. $\frac{1}{2}\left\langle\left(\dot{\ell}_{t}\right)^{2}, \nabla \bar{F}_{r}\left(\ell_{t}\right)\right\rangle \leq-\left\langle\dot{\ell}_{t}, \mathbf{H}\left[\bar{F}_{r}\left(\ell_{t}\right)\right] \dot{\ell}_{t}\right\rangle \leq \frac{3}{2}\left\langle\left(\dot{\ell}_{t}\right)^{2}, \nabla \bar{F}_{r}\left(\ell_{t}\right)\right\rangle$.

Proof of Claim 7.11. We observe that the first summand in (7.28) is exactly $\left\langle\left(\dot{\ell}_{t}\right)^{2}, \nabla \bar{F}_{r}(\ell)\right\rangle$. The claim is thus proved by showing that the second summand has absolute value bounded above by $\frac{1}{2}\left\langle\left(\dot{\ell}_{t}\right)^{2}, \nabla \bar{F}_{r}\left(\ell_{t}\right)\right\rangle$. We accomplish this by breaking this summand into various pieces.

To begin, we focus on the terms in this summand where $j=r$.

Let $K_{r} \subseteq[r-1]$ be the set of indices where $\left|2 \dot{\ell}_{t}^{i} \exp \left(-\ell_{t}^{i}\right) Y_{i r}\left(\ell_{t}\right)\right| \leq(1 / 2 r)\left|\dot{\ell}_{t}^{r} Y_{r}\left(\ell_{t}\right)\right|$. Summing over the elements in $K_{r}$, we find that

$$
\left|\sum_{i \in K_{r}} 2 \dot{\ell}_{t}^{i} \dot{\ell}_{t}^{r} \exp \left(-\ell_{t}^{i}-\ell_{t}^{r}\right) Y_{i r}\left(\ell_{t}\right)\right| \leq \frac{1}{2}\left(\dot{\ell}_{t}^{r}\right)^{2} \exp \left(-\ell_{t}^{r}\right) Y_{r}\left(\ell_{t}\right) .
$$

From the definition of $L_{r}$ we have $24 r \exp \left(-L_{r}\right) \leq 1 / 12$. Thus if $i<r$ and $i \notin K_{r}$ as $\ell_{t}^{i} \geq L_{r}$ and $Y_{i r}\left(\ell_{t}\right)<3 \max \left\{Y_{i}\left(\ell_{t}\right), Y_{r}\left(\ell_{t}\right)\right\}$ from (7.18), we have that

$$
2\left|\dot{\ell}_{t}^{r} Y_{i r}\left(\ell_{t}\right)\right| \leq 6\left|\dot{\ell}_{t}^{r} Y_{r}\left(\ell_{t}\right)\right| \leq\left|24 r \dot{\ell}_{t}^{i} \exp \left(-\ell_{t}^{i}\right) Y_{i r}\left(\ell_{t}\right)\right| \leq 1 / 12\left|\dot{\ell}_{t}^{i} Y_{i r}\left(\ell_{t}\right)\right| \leq 1 / 4\left|\dot{\ell}_{t}^{i} Y_{i}\left(\ell_{t}\right)\right| \text {. }
$$

Thus for $i<r$ and $i \notin K_{r}$ we have that

$$
\left|2 \dot{\ell}_{t}^{i} \dot{\ell}_{t}^{r} \exp \left(-\ell_{t}^{i}-\ell_{t}^{r}\right) Y_{i r}\left(\ell_{t}\right)\right| \leq\left|2 \dot{\ell}_{t}^{i} \dot{\ell}_{t}^{r} \exp \left(-\ell_{t}^{i}\right) Y_{i r}\left(\ell_{t}\right)\right| \leq \frac{1}{4}\left(\dot{\ell}_{t}^{i}\right)^{2} \exp \left(-\ell_{t}^{i}\right) Y_{i}\left(\ell_{t}\right) .
$$

Next we turn our attention to the terms where $j<r$. 
For $i \in[r-1]$ we let $K_{i} \subseteq[r-1]$ be the set of indices where $\left|\dot{\ell}_{t}^{i}\right|>\left|\dot{\ell}_{t}^{j}\right|$ or where $\left|\dot{\ell}_{t}^{i}\right|=\left|\dot{\ell}_{t}^{j}\right|$ and $j>i$. We observe that for any distinct pair of indices $i, j \in$ [r-1] either $j \in K_{i}$ and $i \notin K_{j}$ or $i \in K_{j}$ and $j \notin K_{i}$. From the definition of $L_{r}$ we have $2 \exp \left(-L_{r}\right) \leq 1 / 12 r$. Hence as $Y_{i j}\left(\ell_{t}\right)<3 Y_{i}\left(\ell_{t}\right)$ from (7.18), we find that $2 \exp \left(-\ell_{t}^{j}\right) Y_{i j}\left(\ell_{t}\right) \leq(1 / 4 r) Y_{i}\left(\ell_{t}\right)$ for $j \in[r-1]$. Therefore, summing over the indices in $K_{i}$ we find that

$$
\left|\sum_{j \in K_{i}} 2 \dot{\ell}_{t}^{i} \dot{\ell}_{t}^{j} \exp \left(-\ell_{t}^{i}-\ell_{t}^{j}\right) Y_{i j}\left(\ell_{t}\right)\right| \leq \frac{1}{4}\left(\dot{\ell}_{t}^{i}\right)^{2} \exp \left(-\ell_{t}^{i}\right) Y_{i}\left(\ell_{t}\right) .
$$

Rearranging the terms and using (7.29), (7.31) and (7.32), we find that

$$
\begin{aligned}
\left|\sum_{i=1}^{r-1} \sum_{j=i+1}^{r} 2 \dot{\ell}_{t}^{i} \dot{\ell}_{t}^{j} \exp \left(-\ell_{t}^{i}-\ell_{t}^{j}\right) Y_{i j}\left(\ell_{t}\right)\right| \leq & \left|\sum_{i=1}^{r-1} \sum_{j \in K_{i}} 2 \dot{\ell}_{t}^{i} \dot{\ell}_{t}^{j} \exp \left(-\ell_{t}^{i}-\ell_{t}^{j}\right) Y_{i j}\left(\ell_{t}\right)\right| \\
& +\left|\sum_{i \in K_{r}} 2 \dot{\ell}_{t}^{i} \dot{\ell}_{t}^{r} \exp \left(-\ell_{t}^{i}-\ell_{t}^{r}\right) Y_{i r}\left(\ell_{t}\right)\right| \\
& +\left|\sum_{i \notin K_{r}} 2 \dot{\ell}_{t}^{i} \dot{\ell}_{t}^{r} \exp \left(-\ell_{t}^{i}-\ell_{t}^{r}\right) Y_{i r}\left(\ell_{t}\right)\right| \\
\leq & \sum_{i=1}^{r-1} \frac{1}{4}\left(\dot{\ell}_{t}^{i}\right)^{2} \exp \left(-\ell_{t}^{i}\right) Y_{i}\left(\ell_{t}\right) \\
& +\frac{1}{2}\left(\dot{\ell}_{t}^{r}\right)^{2} \exp \left(-\ell_{t}^{r}\right) Y_{r}\left(\ell_{t}\right) \\
& +\sum_{i \notin K_{r}} \frac{1}{4}\left(\dot{\ell}_{t}^{i}\right)^{2} \exp \left(-\ell_{t}^{i}\right) Y_{i}\left(\ell_{t}\right) \\
\leq & \frac{1}{2}\left\langle\left(\dot{\ell}_{t}\right)^{2}, \nabla \bar{F}_{r}\left(\ell_{t}\right)\right\rangle .
\end{aligned}
$$

As explained above, the claim now follows.

Thus, applying Claim 7.11 and by focusing on the term in $\left\langle\left(\dot{\ell}_{t}\right)^{2}, \nabla \bar{F}_{r}\left(\ell_{t}\right)\right\rangle$ corresponding to $\ell_{t}^{1}$ and tossing out the rest—which are all non-negative-we get our desired bound:

$$
-\left\langle\dot{\ell}_{t}, \mathbf{H}\left[\bar{F}_{r}\left(\ell_{t}\right)\right] \dot{\ell}_{t}\right\rangle \geq \frac{1}{2}\left\langle\left(\dot{\ell}_{t}\right)^{2}, \nabla \bar{F}_{r}\left(\ell_{t}\right)\right\rangle \geq \frac{1}{2}\left(\dot{\ell}_{t}^{1}\right)^{2} \exp \left(-\ell_{t}^{1}\right) Y_{1}\left(\ell_{t}\right) \geq \frac{1}{2} \exp (-t) .
$$

For the last inequality, recall from (7.12) that $1<Y_{1}\left(\ell_{t}\right)$. Combining (7.33) with our previous bound on $\left\langle\ell_{t}, \nabla \bar{F}_{r}\left(\ell_{t}\right)\right\rangle$ from (7.27), we see that

$$
\left\|\left(\ell_{t}, \dot{\ell}_{t}\right)\right\|_{\mathfrak{h}, \mathcal{R}_{r}}^{2}=\frac{-\left\langle\dot{\ell}_{t}, \mathbf{H}\left[\bar{F}_{r}\left(\ell_{t}\right)\right] \dot{\ell}_{t}\right\rangle}{\left\langle\ell_{t}, \nabla \bar{F}_{r}\left(\ell_{t}\right)\right\rangle} \geq \frac{1}{2\left(B_{1} t+B_{2}\right)} .
$$

Hence the length of this path in the entropy metric is at least

$$
\int_{\ell_{0}^{1}}^{\ell_{1}^{1}} \sqrt{\frac{1}{2\left(B_{1} t+B_{2}\right)}} d t=\frac{1}{2 \sqrt{2} B_{1}}\left(\sqrt{B_{1} \ell_{1}^{1}+B_{2}}-\sqrt{B_{1} \ell_{0}^{1}+B_{2}}\right) .
$$


Before we can apply Lemma 7.10 to show that $\left(\mathcal{M}^{1}\left(\mathcal{R}_{r}\right), d_{\mathfrak{h}, \mathcal{R}_{r}}\right)$ has infinite diameter, we require two more estimates. The first states that for a length function in $\mathcal{M}^{1}\left(\mathcal{R}_{r}\right)$ when $\ell^{r}$ is bounded from below, there is an upper bound on the length of the shortest edge that is not $r$.

LEMMA 7.12. Let $r \geq 2$. If $\ell \in \mathcal{M}^{1}\left(\mathcal{R}_{r}\right)$ where $\ell^{r} \geq \log (3)$, then $\min \left\{\ell^{i} \mid i \in[r-1]\right\} \leq$ $\log (4 r-5)$.

Proof. We first prove the lemma under the additional assumption that $\ell^{i}=\ell^{1}$ for any $i \in[r-1]$. In this case, we have that $\ell^{i}=\log (L)$ for $i \in[r-1]$ and some $L>2 r-3$. By Lemma 7.7, we have

$$
\log (3) \leq \ell^{r}=\log \left(\frac{L+(2 r-1)}{L-(2 r-3)}\right) .
$$

Hence we have that $3(L-(2 r-3)) \leq L+(2 r-1)$, which implies that $L \leq 4 r-5$.

Next we prove the general case. Let $\ell \in \mathcal{M}^{1}\left(\mathcal{R}_{r}\right)$ be such that $\ell^{r} \geq \log (3)$. Without loss of generality, we assume that $\ell^{1}=\min \left\{\ell^{i} \mid i \in[r-1]\right\}$.

If $\ell^{1} \leq \log (2 r-3)$, then we are done.

Otherwise, we may decrease the lengths $\ell^{2}, \ldots, \ell^{r-1}$ to be equal to $\ell^{1}$ while increasing $\ell^{r}$ to maintain the fact that the metric has unit entropy. The assumption that $\ell^{1}>\log (2 r-3)$ ensures that $\ell^{r}$ is finite. Denote the resulting metric by $\hat{\ell}$. Observe that $\hat{\ell}^{r} \geq \ell^{r} \geq \log (3)$. By the special case considered above, $\hat{\ell}^{i} \leq \log (4 r-5)$ for each $i \in[r-1]$. As $\ell^{1}=\hat{\ell}^{1}$, this completes the proof of the lemma.

The second estimate shows that when the length of an edge is small for a length function in $\mathcal{M}^{1}\left(\mathcal{R}_{r}\right)$, the lengths of the other edges must be very large.

LEMMA 7.13. Let $r \geq 2$ and let $\ell \in \mathcal{M}^{1}\left(\mathcal{R}_{r}\right)$. For any $\epsilon>0$, if $\ell^{i} \leq \epsilon$ for some $i \in[r]$, then for any $j \in[r]-\{i\}$ we have $\ell^{j}>-\log (\exp (\epsilon)-1)$.

Proof. The subrose consisting of the edges $i$ and $j$ has entropy less than or equal to 1 (strictly less than 1 when $r \geq 3$ ). By Lemma 7.7, this implies that

$$
\ell^{j} \geq \log \left(\frac{\exp \left(\ell^{i}\right)+3}{\exp \left(\ell^{i}\right)-1}\right)>-\log \left(\exp \left(\ell^{i}\right)-1\right) \geq-\log (\exp (\epsilon)-1) .
$$

We can now prove the main inequality in this section that shows that any path that shrinks the length of an edge to zero must have infinite length.

Proposition 7.14. Let $r \geq 2$. For any $D>0$, there is an $\epsilon>0$ such that for any $\ell \in$ $\mathcal{M}^{1}\left(\mathcal{R}_{r}\right)$ with $\min \left\{\ell^{i} \mid i \in[r]\right\} \leq \epsilon$ we have $d_{\mathfrak{h}, \mathcal{R}_{r}}(\log (2 r-1) \cdot \mathbf{1}, \ell) \geq D$.

Proof. Let $L_{0}=\max \left\{\log (4 r-5), L_{r}\right\}$, where $L_{r}$ is the constant from Lemma 7.10. Fix an $\epsilon>0$ such that

$$
\frac{1}{2 \sqrt{2} B_{1}}\left(\sqrt{-B_{1} \log (\exp (\epsilon)-1)+B_{2}}-\sqrt{B_{1} L_{0}+B_{2}}\right) \geq D .
$$


Since $-\log (x-1) \rightarrow \infty$ as $x \rightarrow 1^{+}$, such an $\epsilon$ exists. Observe that for this $\epsilon$ we have that $-\log (\exp (\epsilon)-1) \geq L_{0}$.

Let $\ell \in \mathcal{M}^{1}\left(\mathcal{R}_{r}\right)$ be such that $\min \left\{\ell^{i} \mid i \in[r]\right\} \leq \epsilon$ and let $\ell_{t}:[0,1] \rightarrow \mathcal{M}^{1}\left(\mathcal{R}_{r}\right)$ be a piecewise smooth path where $\ell_{0}=\log (2 r-1) \cdot \mathbf{1}$ and $\ell_{1}=\ell$. We will show that the entropy length of this path is at least $D$. As the path is arbitrary, this shows that $d_{\mathfrak{h}, \mathcal{R}_{r}}(\log (2 r-1) \cdot \mathbf{1}, \ell) \geq D$ as desired.

Without loss of generality, assume that $\ell^{r}=\min \left\{\ell^{i} \mid i \in[r]\right\}$. Let $\delta_{0} \in[0,1]$ be the minimal value so that $\ell_{t}^{r}=\min \left\{\ell_{t}^{i} \mid i \in[r], t \in\left[\delta_{0}, 1\right]\right\}$. We observe that $\ell_{\delta_{0}}^{r} \geq \log (3)$. Indeed, there is another index $i \in[r-1]$ such that $\ell_{\delta_{0}}^{i}=\ell_{\delta_{0}}^{r}$. If $\ell_{\delta_{0}}^{r}<\log (3)$, then the entropy of the subgraph consisting of the $i$ th and $r$ th edges is greater than 1 . This contradicts the fact that the entropy of $\ell_{\delta_{0}}$ is equal to 1 .

As there is an automorphism of the $r$-rose permuting any two edges, by redefining $\ell_{t}$ if necessary, we may assume that $\ell_{t}^{1}=\min \left\{\ell_{t}^{i} \mid i \in[r-1]\right\}$ for each $t \in\left[\delta_{0}, 1\right]$. By Lemma 7.12, as $\ell_{\delta_{0}}^{r} \geq \log (3)$, we have that $\ell_{\delta_{0}}^{1} \leq \log (4 r-5) \leq L_{0}$. By Lemma 7.13, as $\ell_{1}^{r} \leq \epsilon$, we have that $\ell_{1}^{1}>-\log (\exp (\epsilon)-1) \geq L_{0}$.

Let $\delta_{1} \in\left[\delta_{0}, 1\right]$ be the minimal value so that $\ell_{t}^{1} \geq L_{0}$ for all $t \in\left[\delta_{1}, 1\right]$. As $\mathcal{L}_{\mathfrak{h}, \mathcal{R}_{r}}\left(\ell_{t} \mid[0,1]\right) \geq \mathcal{L}_{\mathfrak{h}, \mathcal{R}_{r}}\left(\ell_{t} \mid\left[\delta_{1}, 1\right]\right)$, it suffices to show that the latter is bounded below by $D$.

By only considering the portion of $\ell_{t}$ along the subintervals of $\left[\delta_{1}, 1\right]$ with $\dot{\ell}_{t}^{1}>0$, we find by Lemma 7.10 that

$$
\mathcal{L}_{\mathfrak{h}, \mathcal{R}_{r}}\left(\ell_{t} \mid\left[\delta_{1}, 1\right]\right) \geq \frac{1}{2 \sqrt{2} B_{1}}\left(\sqrt{B_{1} \ell_{1}^{1}+B_{2}}-\sqrt{B_{1} \ell_{\delta_{1}}^{1}+B_{2}}\right) .
$$

As $\ell_{1}^{r} \leq \epsilon$, we have $\ell_{1}^{1} \geq-\log (\exp (\epsilon)-1)$ by Lemma 7.13. By definition $\ell_{\delta_{1}}^{1}=L_{0}$. Therefore

$$
\mathcal{L}_{\mathfrak{h}, \mathcal{R}_{r}}\left(\ell_{t} \mid\left[\delta_{1}, 1\right]\right) \geq \frac{1}{2 \sqrt{2} B_{1}}\left(\sqrt{-B_{1} \log (\exp (\epsilon)-1)+B_{2}}-\sqrt{B_{1} L_{0}+B_{2}}\right) \geq D .
$$

\section{Proof of Theorem 1.1}

In this section we give the proof of the first main result of this paper. Theorem 1.1 states that $\left(\mathcal{X}^{1}\left(\mathbb{F}_{r}\right), d_{\mathfrak{h}}\right)$ is complete when $r=2$ and not complete if $r \geq 3$.

Proof of Theorem 1.1. In $\S 6$ we showed that $\left(X^{1}\left(\mathbb{F}_{2}\right), d_{\mathfrak{h}}\right)$ is complete (Proposition 6.8), and so it remains to show that $\left(\mathcal{X}^{1}\left(\mathbb{F}_{r}\right), d_{\mathfrak{h}}\right)$ is not complete when $r \geq 3$. This is a simple consequence of Proposition 7.8, as we now explain.

Let $r \geq 3$ and let $\ell_{t}:[0,1) \rightarrow \mathcal{M}^{1}\left(\mathcal{R}_{r}\right)$ be the path described in Proposition 7.8. Using the natural homeomorphism $\mathcal{M}^{1}\left(\mathcal{R}_{r}\right) \leftrightarrow \mathcal{X}^{1}\left(\mathcal{R}_{r}\right.$, id), we can consider $\ell_{t}$ as a path in $X^{1}\left(\mathbb{F}_{r}\right)$.

The sequence of length functions $\left(\ell_{1-1 / n}\right)_{n \in \mathbb{N}}$ is a Cauchy sequence in $\left(\mathcal{X}^{1}\left(\mathbb{F}_{r}\right), d_{\mathfrak{h}}\right)$ as the entropy distance on $\mathcal{M}^{1}\left(\mathcal{R}_{r}\right)$ is an upper bound on the entropy distance on $\mathcal{X}^{1}\left(\mathbb{F}_{r}\right)$.

We claim that this sequence does not have a limit. Indeed, any length function $\ell$ that does not lie in $\mathcal{X}^{1}\left(\mathcal{R}_{r}\right.$, id) has an open neighborhood in the weak topology that does not intersect $\mathcal{X}^{1}\left(\mathcal{R}_{r}, \mathrm{id}\right)$. As the metric topology and the weak topology agree, any possible limit of this sequence must lie in $\mathcal{X}^{1}\left(\mathcal{R}_{r}, \mathrm{id}\right)$. However, as $\ell_{1-1 / n}^{r} \rightarrow \infty$ as $n \rightarrow \infty$, we 
see that for any $\ell \in \mathcal{X}^{1}\left(\mathcal{R}_{r}, \mathrm{id}\right)$, there is an open neighborhood of $\ell$ in the weak topology $U \subset \mathcal{X}^{1}\left(\mathbb{F}_{r}\right)$ such that $\ell_{1-1 / n} \in U$ for only finitely many $n$. Hence, again as the metric topology and the weak topology agree, we see that this sequence does not have a limit in $\chi^{1}\left(\mathcal{R}_{r}, \mathrm{id}\right)$.

9. The completion of $\left(\mathcal{M}^{1}\left(\mathcal{R}_{r}\right), d_{\mathfrak{h}, \mathcal{R}_{r}}\right)$

The goal of this section is to prove Theorem 1.2 which states that the completion of $\left(\mathcal{M}^{1}\left(\mathcal{R}_{r}\right), d_{\mathfrak{h}, \mathcal{R}_{r}}\right)$ is homeomorphic to the complement of the vertices in an $(r-1)$-simplex. Intuitively, the newly added completion points correspond to unit-entropy metrics on the subroses of $\mathcal{R}_{r}$ consisting of at least two edges. Specifically, a face of dimension $k-1$ corresponds to unit-entropy metrics on a sub- $k$-rose. We observe that $\mathcal{R}_{1}$ does not possess a metric with unit entropy. This accounts for the missing vertices in the completion.

There are two steps to the proof. First, in $\$ 9.1$ we introduce a model space $\widehat{\mathcal{M}}^{1}\left(\mathcal{R}_{r}\right)$ for the completion of $\mathcal{M}^{1}\left(\mathcal{R}_{r}\right)$ with respect to the entropy metric. This model considers $\mathcal{M}\left(\mathcal{R}_{r}\right)$ as a subset of $[0, \infty]^{r}$ and adds the faces where at most $r-2$ of the coordinates are equal to $\infty$. It is apparent from the construction that $\widehat{\mathcal{M}}^{1}\left(\mathcal{R}_{r}\right)$ is homeomorphic to the complement of the vertices in an $(r-1)$-simplex. Proposition 9.6 shows that the distance function $d_{\mathfrak{h}, \mathcal{R}_{r}}$ on $\mathcal{M}^{1}\left(\mathcal{R}_{r}\right)$ extends to a distance function on $\widehat{\mathcal{M}}^{1}\left(\mathcal{R}_{r}\right)$. It is clear that $\mathcal{M}^{1}\left(\mathcal{R}_{r}\right)$ is dense in $\widehat{\mathcal{M}}^{1}\left(\mathcal{R}_{r}\right)$. In $\S 9.2$ we complete the proof of Theorem 1.2 by showing that $\left(\widehat{\mathcal{M}}^{1}\left(\mathcal{R}_{r}\right), d_{\mathfrak{h}, \mathcal{R}_{r}}\right)$ is complete. Example 9.7 illustrates $\widehat{\mathcal{M}}^{1}\left(\mathcal{R}_{3}\right)$ and contrasts this with the closure of $C V\left(\mathcal{R}_{3}\right.$, id) considered as a subset of $\mathbb{R}^{\mathbb{F}_{3}}$ in the axis topology.

Finally, in $\$ 9.3$ we show that the diameter of cross-section of $\mathcal{M}^{1}\left(\mathcal{R}_{r}\right)$ consisting of length functions with $\ell^{i}=\epsilon$ for some fixed $i \in[r]$ and $\epsilon>0$ goes to zero as $\epsilon \rightarrow 0^{+}$. This is important for $\S 11$ where we show that $\mathcal{X}^{1}\left(\mathcal{R}_{r}\right.$, id $)$ is a bounded subset of $\left(\mathcal{X}^{1}\left(\mathbb{F}_{r}\right), d_{\mathfrak{h}}\right)$.

9.1. The model space $\widehat{\mathcal{M}}^{1}\left(\mathcal{R}_{r}\right)$. In this section we introduce a model for the completion of $\left(\mathcal{M}^{1}\left(\mathcal{R}_{r}\right), d_{\mathfrak{h}, \mathcal{R}_{r}}\right)$. As mentioned above, we add the faces to $\mathcal{M}^{1}\left(\mathcal{R}_{r}\right)$ corresponding to subroses consisting of at least two edges where the rest of the edge lengths are infinite.

Topologize $[0, \infty]$ as a closed interval. The natural inclusion $\mathcal{M}^{1}\left(\mathcal{R}_{r}\right) \subset(0, \infty)^{r} \subset$ $[0, \infty]^{r}$ is an embedding. By setting $x+\infty=\infty$ and $\exp (-\infty)=0$ we get that the functions $\bar{F}_{r}$ from (7.5), $X_{i}$ from (7.2), $Y_{i}$ from (7.7), $X_{i j}$ from (7.16) and $Y_{i j}$ from (7.17) extend to continuous functions on $[0, \infty]^{r}$, and the entropy function $\mathfrak{h}_{r}(\ell)$ extends to $(0, \infty]^{r}$.

Given a subset $S \subseteq[r]$, we identify the subsets

$$
\begin{aligned}
& \mathcal{M}_{S}=\left\{\ell \in(0, \infty]^{r} \mid \ell^{i}<\infty \text { if } i \in S \text { and } \ell^{i}=\infty \text { if } i \notin S\right\}, \quad \text { and } \\
& \mathcal{M}_{S}^{1}=\left\{\ell \in \mathcal{M}_{S} \mid \bar{F}_{r}(\ell)=0\right\} .
\end{aligned}
$$

Notice that $\mathcal{M}_{[r]}=\mathcal{M}\left(\mathcal{R}_{r}\right)$ and that $\mathcal{M}_{S} \cap \mathcal{M}_{S^{\prime}}=\emptyset$ if $S \neq S^{\prime}$. We further observe that $\mathcal{M}_{\emptyset}^{1}=\emptyset$ and $\mathcal{M}_{\{i\}}^{1}=\emptyset$ for any $i \in[r]$. For the latter, note that $X_{i}(\ell)=1$ and $Y_{i}(\ell)=1$ for any $\ell \in \mathcal{M}_{\{i\}}$. Thus for $\ell \in \mathcal{M}_{\{i\}}$ we have that $\bar{F}_{r}(\ell)=1-\exp \left(-\ell^{i}\right)>0$. 
Fix $S \subseteq[r]$ with $|S|>1$ and let $\iota_{S}: S \rightarrow\{1,2, \ldots,|S|\}$ be the order-preserving bijection and let $\varepsilon_{S}:[0, \infty]^{|S|} \rightarrow[0, \infty]^{r}$ be the embedding defined by

$$
\varepsilon_{S}\left(\ell^{1}, \ldots, \ell^{|S|}\right)^{i}= \begin{cases}\ell^{\iota_{S}(i)} & \text { if } i \in S, \\ \infty & \text { otherwise }\end{cases}
$$

The function $\varepsilon_{S}$ allows us to consider a length function on $\mathcal{R}_{|S|}$ as a length function on $\mathcal{R}_{r}$ where the edges not in $S$ have infinite length. Indeed, with these definitions we have $\varepsilon_{S}\left(\mathcal{M}\left(\mathcal{R}_{|S|}\right)\right)=\mathcal{M}_{S}$. The following lemma is immediate from Proposition 7.3 and the definitions.

LEMMA 9.1. Let $r \geq 2$ and let $S \subseteq[r]$ have $|S|>1$. Then the following statements are true.

(1) $\bar{F}_{r} \circ \varepsilon_{S}=\bar{F}_{|S|}$.

(2) For $\ell \in \mathcal{M}\left(\mathcal{R}_{|S|}\right)$, we have $\mathfrak{h}_{r}\left(\varepsilon_{S}(\ell)\right)=\mathfrak{h}_{|S|}(\ell)$.

(3) $\varepsilon_{S}$ restricts to a homeomorphism $\mathcal{M}^{1}\left(\mathcal{R}_{|S|}\right) \rightarrow \mathcal{M}_{S}^{1}$.

Next we define the sets

$$
\begin{aligned}
\widehat{\mathcal{M}}\left(\mathcal{R}_{r}\right) & =(0, \infty]^{r}=\bigcup_{S \subseteq[r]} \mathcal{M}_{S}, \\
\widehat{\mathcal{M}}^{1}\left(\mathcal{R}_{r}\right) & =\left\{\ell \in \widehat{\mathcal{M}}\left(\mathcal{R}_{r}\right) \mid \bar{F}_{r}(\ell)=0\right\}=\bigcup_{S \subseteq[r]} \mathcal{M}_{S}^{1} .
\end{aligned}
$$

The set $\widehat{\mathcal{M}}^{1}\left(\mathcal{R}_{r}\right)$ is homeomorphic to the complement of the set of vertices in an $(r-1)$-simplex. Applying Proposition 7.5, Lemma 9.1, and the above definitions, we get the following result.

Proposition 9.2. Let $r \geq 2$. A length function $\ell \in \widehat{\mathcal{M}}\left(\mathcal{R}_{r}\right)$ lies in $\widehat{\mathcal{M}}^{1}\left(\mathcal{R}_{r}\right)$ if and only if it has entropy equal to 1.

As $Y_{i}(\ell)$ and $Y_{i j}(\ell)$ extend to continuous functions on $\widehat{\mathcal{M}}\left(\mathcal{R}_{r}\right)$, using the expressions for the partial derivatives of $\bar{F}_{r}(\ell)$ in (7.10), (7.20) and (7.21), we see that $\partial_{i} \bar{F}_{r}(\ell), \partial_{i i} \bar{F}_{r}(\ell)$ and $\partial_{i j} \bar{F}_{r}(\ell)$ extend to continuous functions on $\widehat{\mathcal{M}}\left(\mathcal{R}_{r}\right)$. Using these formulas, we see that extensions satisfy the following properties.

LEMmA 9.3. Let $r \geq 2$, let $S \subseteq[r]$ have $|S|>1$ and fix $\ell \in \mathcal{M}\left(\mathcal{R}_{|S|}\right)$. Then for $i, j \in[r]$ the following hold:

$$
\begin{aligned}
\partial_{i} \bar{F}_{r}\left(\varepsilon_{S}(\ell)\right) & = \begin{cases}\partial_{\iota_{S}(i)} \bar{F}_{|S|}(\ell) & \text { if } i \in S, \\
0 & \text { otherwise; }\end{cases} \\
\partial_{i j} \bar{F}_{r}\left(\varepsilon_{S}(\ell)\right) & = \begin{cases}\partial_{l_{S}(i) \iota_{S}(j)} \bar{F}_{|S|}(\ell) & \text { if } i, j \in S, \\
0 & \text { otherwise. }\end{cases}
\end{aligned}
$$

Hence both $\nabla \bar{F}_{r}(\ell)$ and $\mathbf{H}\left[\bar{F}_{r}(\ell)\right]$ are well defined for $\ell \in \widehat{\mathcal{M}}\left(\mathcal{R}_{r}\right)$. Additionally, the inner product $\left\langle\ell, \nabla \bar{F}_{r}(\ell)\right\rangle$ extends continuously as we next show. 
LEMMA 9.4. Let $r \geq 2$. The function $\ell \mapsto\left\langle\ell, \nabla \bar{F}_{r}(\ell)\right\rangle$ has a continuous extension to $\widehat{\mathcal{M}}^{1}\left(\mathcal{R}_{r}\right)$. Moreover, if $S \subseteq[r]$ has $|S|>1$ and $\ell \in \mathcal{M}_{|S|}$, then

$$
\left\langle\varepsilon_{S}(\ell), \nabla \bar{F}_{r}\left(\varepsilon_{S}(\ell)\right)\right\rangle=\left\langle\ell, \nabla \bar{F}_{|S|}(\ell)\right\rangle .
$$

Proof. Both of these statements follow from the expressions for the partial derivatives of $\bar{F}_{r}(\ell)$ in (7.10) as $x \exp (-x) \rightarrow 0$ when $x \rightarrow \infty$.

We define the tangent bundle $T \widehat{\mathcal{M}}^{1}\left(\mathcal{R}_{r}\right)$ as the subspace of $(\ell, \mathbf{v}) \in \widehat{\mathcal{M}}^{1}\left(\mathcal{R}_{r}\right) \times \mathbb{R}^{r}$ where $\left\langle\mathbf{v}, \nabla \bar{F}_{r}(\ell)\right\rangle=0$. The subset of $(\ell, \mathbf{v}) \in T \widehat{\mathcal{M}}^{1}\left(\mathcal{R}_{r}\right)$ where $\ell \in \mathcal{M}_{S}^{1}$ is denoted by $T \mathcal{M}_{S}^{1}$. For consistency, we denote $T \mathcal{M}_{[r]}^{1}$ by $T \mathcal{M}^{1}\left(\mathcal{R}_{r}\right)$. There is an embedding $\boldsymbol{\varepsilon}_{S}: \mathbb{R}^{|S|} \rightarrow \mathbb{R}^{r}$ defined by

$$
\boldsymbol{\varepsilon}_{S}\left(\mathbf{v}^{1}, \ldots, \mathbf{v}^{|S|}\right)^{i}= \begin{cases}\mathbf{v}^{\iota_{S}(i)} & \text { if } i \in S, \\ 0 & \text { otherwise. }\end{cases}
$$

This allows us to define an embedding $T_{S}: T \mathcal{M}^{1}\left(\mathcal{R}_{|S|}\right) \rightarrow T \widehat{\mathcal{M}}^{1}\left(\mathcal{R}_{r}\right)$ whose image is contained in $T \mathcal{M}_{S}^{1}$ by $T_{S}(\ell, \mathbf{v})=\left(\varepsilon_{S}(\ell), \boldsymbol{\varepsilon}_{S}(\mathbf{v})\right)$. Proposition 4.14, together with Lemmas 9.3 and 9.4 , has the following implication.

PROpOSITION 9.5. Let $r \geq 2$. The entropy norm $\|\cdot\|_{\mathfrak{h}, \mathcal{R}_{r}}: T \mathcal{M}^{1}\left(\mathcal{R}_{r}\right) \rightarrow \mathbb{R}$ extends to a continuous semi-norm $\|\cdot\|_{\mathfrak{h}, \mathcal{R}_{r}}: T \widehat{\mathcal{M}}^{1}\left(\mathcal{R}_{r}\right) \rightarrow \mathbb{R}$. Moreover, the embedding maps $T_{S}: T \mathcal{M}^{1}\left(\mathcal{R}_{|S|}\right) \rightarrow T \widehat{\mathcal{M}}^{1}\left(\mathcal{R}_{r}\right)$ are norm-preserving. Specifically, if $S \subseteq[r]$ has $|S|>1$, and $(\ell, \mathbf{v})=T_{S}\left(\ell_{S}, \mathbf{v}_{S}\right)$, then the extension satisfies

$$
\|(\ell, \mathbf{v})\|_{\mathfrak{h}, \mathcal{R}_{r}}=\left\|\left(\ell_{S}, \mathbf{v}_{S}\right)\right\|_{\mathfrak{h}, \mathcal{R}_{|S|}} .
$$

The reason why the extension fails to be a norm is as follows. If $(\ell, \mathbf{v}) \in \mathcal{M}_{S}^{1} \times \mathbb{R}^{r}$ and $\mathbf{v}^{i}=0$ for $i \in S$, then $(\ell, \mathbf{v}) \in T \mathcal{M}_{S}^{1}$ as $\partial_{i} \bar{F}_{r}(\ell)=0$ whenever $i \notin S$. Further, $\partial_{i j} \bar{F}_{r}(\ell)=0$ whenever $i \notin S$ or $j \notin S$. Thus $-\left\langle\mathbf{v}, \mathbf{H}\left[\bar{F}_{r}\right](\ell) \mathbf{v}\right\rangle=0$ and hence $\|(\ell, \mathbf{v})\|_{\mathfrak{h}, \mathcal{R}_{r}}=0$ for such points.

The following proposition is the main result of this section. It shows that $\widehat{\mathcal{M}}^{1}\left(\mathcal{R}_{r}\right)$ is contained in the completion of $\left(\mathcal{M}^{1}\left(\mathcal{R}_{r}\right), d_{\mathfrak{h}, \mathcal{R}_{r}}\right)$.

PROPOSITION 9.6. Let $r \geq 2$. The following statements hold.

(1) The extension of the entropy norm defines a distance function $d_{\mathfrak{h}, \mathcal{R}_{r}}$ on $\widehat{\mathcal{M}}^{1}\left(\mathcal{R}_{r}\right)$.

(2) The inclusion $\left(\mathcal{M}^{1}\left(\mathcal{R}_{r}\right), d_{\mathfrak{h}, \mathcal{R}_{r}}\right) \rightarrow\left(\widehat{\mathcal{M}}^{1}\left(\mathcal{R}_{r}\right), d_{\mathfrak{h}, \mathcal{R}_{r}}\right)$ is an isometric embedding.

(3) The topology induced by $d_{\mathfrak{h}, \mathcal{R}_{r}}$ equals the subspace topology $\widehat{\mathcal{M}}^{1}\left(\mathcal{R}_{r}\right) \subseteq[0, \infty]^{r}$.

Proof. By definition $\widehat{\mathcal{M}}^{1}\left(\mathcal{R}_{2}\right)=\mathcal{M}^{1}\left(\mathcal{R}_{2}\right)$. Hence the proposition is obvious for $r=2$. We assume from now on that $r \geq 3$.

First we need to show that for each $\ell, \ell^{\prime} \in \widehat{\mathcal{M}}^{1}\left(\mathcal{R}_{r}\right)$ there is a path $\ell_{t}:[0,1] \rightarrow \widehat{\mathcal{M}}^{1}\left(\mathcal{R}_{r}\right)$ with $\ell_{0}=\ell$ and $\ell_{1}=\ell^{\prime}$ that has finite length so that $d_{\mathfrak{h}, \mathcal{R}_{r}}\left(\ell, \ell^{\prime}\right)$ is defined. Notice that by Proposition 9.5 for each $S \subseteq[r]$ with $|S|>1$, any two points in $\mathcal{M}_{S}^{1}$ can be joined by a path of finite length. Hence it suffices to show that, for any $S \subseteq[r]$ with $|S|>1$, there is a path of finite length joining $\log (2 r-1) \cdot \mathbf{1} \in \mathcal{M}^{1}\left(\mathcal{R}_{r}\right)$ to $\varepsilon_{S}(\log (2|S|-1) \cdot \mathbf{1}) \in \mathcal{M}_{S}^{1}$. 
Recall that in Proposition 7.8 we showed that there is a path $\ell_{t}:[0,1] \rightarrow \widehat{\mathcal{M}}^{1}\left(\mathcal{R}_{r}\right)$ where $\ell_{0}=\log (2 r-1) \cdot \mathbf{1}$ and $\ell_{1}=\varepsilon_{[r-1]}(\log (2 r-3) \cdot \mathbf{1}) \in \mathcal{M}_{[r-1]}^{1}$ that has finite length.

Now, given $S \subseteq[r]$ with $|S|>1$, we inductively define subsets $S_{i}$ for $i=0, \ldots, r-$ $|S|$ by $S_{0}=[r]$, and $S_{i+1}=S_{i}-\left\{\max \left(S_{i}-S\right)\right\}$ so that $S_{r-|S|}=S$. The calculation in Proposition 7.8 shows that there is a finite-length path $\left(\ell_{i}\right)_{t}:[0,1] \rightarrow \widehat{\mathcal{M}}^{1}\left(\mathcal{R}_{r}\right)$ with $\left(\ell_{i}\right)_{0}=\varepsilon_{S_{i}}(\log (2(r-i)-1) \cdot \mathbf{1}) \in \mathcal{M}_{S_{i}}^{1}$ and $\left(\ell_{i}\right)_{1}=\varepsilon_{S_{i+1}}(\log (2(r-(i+1))-$ 1) - 1) $\in \mathcal{M}_{S_{i+1}}^{1}$. The concatenation of these paths is a path with finite length from $\log (2 r-1) \cdot \mathbf{1} \in \mathcal{M}^{1}\left(\mathcal{R}_{r}\right)$ to $\varepsilon_{S}(\log (2|S|-1) \cdot \mathbf{1}) \in \mathcal{M}_{S}^{1}$. Therefore $d_{\mathfrak{h}, \mathcal{R}_{r}}\left(\ell, \ell^{\prime}\right)$ is defined for all $\ell, \ell^{\prime} \in \widehat{\mathcal{M}}^{1}\left(\mathcal{R}_{r}\right)$.

Next, we show that $d_{\mathfrak{h}, \mathcal{R}_{r}}$ defines a distance function on $\widehat{\mathcal{M}}^{1}\left(\mathcal{R}_{r}\right)$. Symmetry and the triangle inequality obviously hold. What needs to be checked is that if $\ell$ and $\ell^{\prime}$ are distinct points in $\widehat{\mathcal{M}}^{1}\left(\mathcal{R}_{r}\right)$, then there is an $\epsilon$ such that any path from $\ell$ to $\ell^{\prime}$ has length at least $\epsilon$.

This is clear if at least one of $\ell$ and $\ell^{\prime}$ lie in $\mathcal{M}^{1}\left(\mathcal{R}_{r}\right)$. Indeed, say $\ell$ lies in $\mathcal{M}^{1}\left(\mathcal{R}_{r}\right)$. Then there is an open set $U \subset \mathcal{M}^{1}\left(\mathcal{R}_{r}\right)$ containing $\ell$ with compact closure $\bar{U}$ such that $\ell^{\prime} \notin \bar{U}$. Further, there is an $\epsilon>0$ such that if $d_{\mathfrak{h}, \mathcal{R}_{r}}\left(\ell, \ell^{\prime \prime}\right)<\epsilon$ then $\ell^{\prime \prime} \in U$. Hence any path from $\ell$ to $\ell^{\prime}$ must have length at least $\epsilon$ and therefore $d_{\mathfrak{h}, \mathcal{R}_{r}}\left(\ell, \ell^{\prime}\right) \geq$ $\epsilon>0$.

It remains to consider the case where neither $\ell$ nor $\ell^{\prime}$ lies in $\mathcal{M}^{1}\left(\mathcal{R}_{r}\right)$. Suppose that there is a sequence of paths $\left(\ell_{n}\right)_{t}:[0,1] \rightarrow \widehat{\mathcal{M}}^{1}\left(\mathcal{R}_{r}\right)$ from $\ell, \ell^{\prime} \in \widehat{\mathcal{M}}^{1}\left(\mathcal{R}_{r}\right)-\mathcal{M}^{1}\left(\mathcal{R}_{r}\right)$ where $\mathcal{L}_{\mathfrak{h}, \mathcal{R}_{r}}\left(\left(\ell_{n}\right)_{t} \mid[0,1]\right) \rightarrow 0$. As the lengths of the paths $\left(\ell_{n}\right)_{t}$ go to 0 , by Proposition 7.14, there is an $\epsilon>0$ such that $\left(\left(\ell_{n}\right)_{t}\right)^{i} \geq \epsilon$ for all $t \in[0,1]$ and all $n$. Hence the images of the paths lie in a compact set of $\widehat{\mathcal{M}}^{1}\left(\mathcal{R}_{r}\right)$ and, by the Arzelà-Ascoli theorem, there is a path $\ell_{t}:[0,1] \rightarrow \widehat{\mathcal{M}}^{1}\left(\mathcal{R}_{r}\right)$ from $\ell$ to $\ell^{\prime}$ with length 0 .

The image of such a path must be contained in $\widehat{\mathcal{M}}^{1}\left(\mathcal{R}_{r}\right)-\mathcal{M}^{1}\left(\mathcal{R}_{r}\right)$. As

$$
\widehat{\mathcal{M}}^{1}\left(\mathcal{R}_{r}\right)-\mathcal{M}^{1}\left(\mathcal{R}_{r}\right)=\bigcup_{S \subset[r]} \mathcal{M}_{S}
$$

we must have that $\dot{\ell}_{t}=0$ since the semi-norm is non-degenerate on any $T \mathcal{M}_{S}^{1}$ by Proposition 9.5 and hence $\ell=\ell^{\prime}$.

This shows (1).

Item (2) follows as any path in $\widehat{\mathcal{M}}^{1}\left(\mathcal{R}_{r}\right)$ with endpoints in $\mathcal{M}^{1}\left(\mathcal{R}_{r}\right)$ is close to a path entirely contained in $\mathcal{M}^{1}\left(\mathcal{R}_{r}\right)$ by continuity of the semi-norm.

Item (3) now follows by continuity of the semi-norm.

9.2. Proof of Theorem 1.2. We can now complete the proof of Theorem 1.2 which states that the completion of $\mathcal{M}^{1}\left(\mathcal{R}_{r}\right)$ with respect to the entropy metric is homeomorphic to the complement of the vertices of an $(r-1)$-simplex. We accomplish this by showing that $\left(\widehat{\mathcal{M}}^{1}\left(\mathcal{R}_{r}\right), d_{\mathfrak{h}, \mathcal{R}_{r}}\right)$ is the completion as we have already observed that this space is homeomorphic to the complement of the vertices of an $(r-1)$-simplex. 
Proof of Theorem 1.2. As the inclusion map $\left(\mathcal{M}^{1}\left(\mathcal{R}_{r}\right), d_{\mathfrak{h}, \mathcal{R}_{r}}\right) \rightarrow\left(\widehat{\mathcal{M}}^{1}\left(\mathcal{R}_{r}\right), d_{\mathfrak{h}, \mathcal{R}_{r}}\right)$ is an isometric embedding by Proposition 9.6(2) and the image is clearly dense, it remains to show that $\left(\widehat{\mathcal{M}}^{1}\left(\mathcal{R}_{r}\right), d_{\mathfrak{h}, \mathcal{R}_{r}}\right)$ is complete.

To this end, let $\left(\ell_{n}\right)_{n \in \mathbb{N}} \subset \widehat{\mathcal{M}}^{1}\left(\mathcal{R}_{r}\right)$ be a Cauchy sequence. Then for each $1 \leq i \leq r$, we have that $\left(\ell^{i}\right)_{n}$ limits to some $\ell_{\infty}^{i} \in[0, \infty]^{r}$ where $\bar{F}_{r}\left(\ell_{\infty}^{1}, \ldots, \ell_{\infty}^{r}\right)=0$. What remains to be shown is that such a limiting length function $\ell_{\infty}$ belongs to $\widehat{\mathcal{M}}^{1}\left(\mathcal{R}_{r}\right)$.

Let $S=\left\{i \in[r] \mid \ell_{\infty}^{i} \neq \infty\right\}$. If $\ell^{i} \neq 0$ for all $i \in[r]$, then $\ell \in \mathcal{M}_{S}^{1} \subset \widehat{\mathcal{M}}^{1}\left(\mathcal{R}_{r}\right)$ and we are done. This is indeed always the case as by Proposition 7.14, the limiting length $\ell_{\infty}^{i}$ is bounded away from zero for all $i$ since the sequence is Cauchy.

Example 9.7. In this example, we compare the completion $\widehat{\mathcal{M}}^{1}\left(\mathcal{R}_{3}\right)$ with the closure of $C V\left(\mathcal{R}_{3}\right.$, id) considered as a subset of $\mathbb{R}^{\mathbb{F}_{3}}$. Recall that $C V\left(\mathcal{R}_{3}, \mathrm{id}\right) \subset C V\left(\mathbb{F}_{3}\right)$ is the space of length functions on $\mathcal{R}_{3}$ with unit volume, that is, the sum of the lengths of the edges is equal to 1 . For the current discussion, the marking is irrelevant. For more information about the closure of $C V\left(\mathbb{F}_{r}\right)$ in $\mathbb{R}^{\mathbb{F}_{r}}$ we refer the reader to the papers by Bestvina and Feighn [7] and Cohen and Lustig [12].

We again consider Figure 5 in $\$ 7.1$, which shows $\mathcal{M}^{1}\left(\mathcal{R}_{r}\right)$ for $r=2$ and $r=3$. These images suggest that as the length of one of the edges goes to infinity, the moduli space $\mathcal{M}^{1}\left(\mathcal{R}_{3}\right)$ limits to the moduli space $\mathcal{M}^{1}\left(\mathcal{R}_{2}\right)$ for the subgraph consisting of the other two edges. There are three such $\mathcal{R}_{2}$ subgraphs in $\mathcal{R}_{3}$, each contributing a one-dimensional face to $\widehat{\mathcal{M}}^{1}\left(\mathcal{R}_{3}\right)$. Figure 6 shows a schematic of $\widehat{\mathcal{M}}^{1}\left(\mathcal{R}_{3}\right)$ contrasted with $\overline{C V\left(\mathcal{R}_{3}, \text { id }\right)}$, the closure of $C V\left(\mathcal{R}_{3}\right.$, id) considered as a subset of $\mathbb{R}^{\mathbb{F}_{3}}$. The spaces are not homeomorphic; $\widehat{\mathcal{M}}^{1}\left(\mathcal{R}_{3}\right)$ is a 2-simplex without vertices, whereas $\overline{C V\left(\mathcal{R}_{3}, \text { id }\right)}$ is a 2 -simplex. A more striking difference comes from the duality between the newly added edges and vertices.

- In $\widehat{\mathcal{M}}^{1}\left(\mathcal{R}_{3}\right)$, as $c \rightarrow \infty$ we obtain a copy of $\mathcal{M}^{1}\left(\mathcal{R}_{2}\right)$ for the subgraph on $a$ and $b$. In $\overline{C V\left(\mathcal{R}_{3}, \mathrm{id}\right)}$, the corresponding sequence would send $a, b \rightarrow 0, c \rightarrow 1$ and the result is a single point corresponding to the graph of groups decomposition of $\langle a, b, c\rangle$ with underlying graph $\mathcal{R}_{1}$ where $\langle a, b\rangle$ is the vertex group and the edge group is trivial.

- In $\widehat{\mathcal{M}}^{1}\left(\mathcal{R}_{3}\right)$, as $b, c \rightarrow \infty$ there is no limit. This is a missing vertex of the 2-simplex; this stems from the fact that $\mathcal{R}_{1}$ cannot be scaled to have unit entropy. In $\overline{C V\left(\mathcal{R}_{3}, \text { id }\right)}$, the corresponding sequence would send $a \rightarrow 0$ and we obtain a one-dimensional face in the closure corresponding to unit-volume length functions on the graph of groups decomposition of $\langle a, b, c\rangle$ with underlying graph $\mathcal{R}_{2}$ where $\langle a\rangle$ is the vertex group and both edge groups are trivial.

9.3. The thin part of $\mathcal{M}^{1}\left(\mathcal{R}_{r}\right)$. For $\epsilon>0$ and $i \in[r]$, we define

$$
\mathcal{S}_{\epsilon}^{i}=\left\{\ell \in \mathcal{M}^{1}\left(\mathcal{R}_{r}\right) \mid \ell^{i}=\epsilon\right\} .
$$

We use the letter ' $S$ ' as we think of this subset as a slice of the moduli space. The goal of this section is to prove the following proposition. 

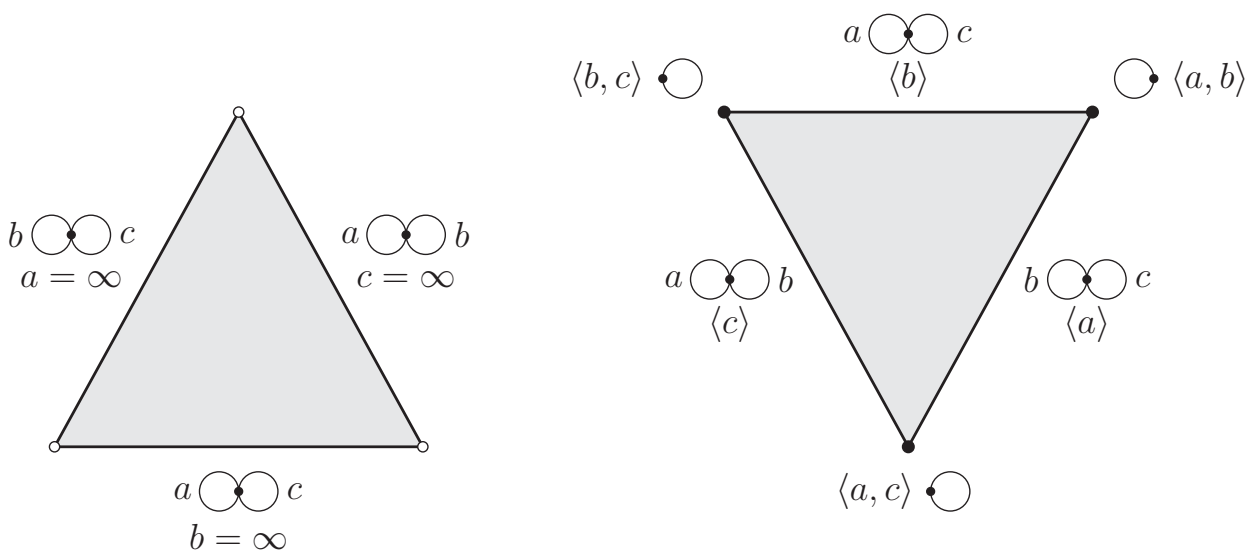

FIGURE 6. The completion of entropy normalization $\widehat{M}^{1}\left(\mathcal{R}_{3}\right)$ contrasted with the closure of the volume normalization $\overline{C V\left(\mathcal{R}_{3}, \text { id }\right)}$ in $\mathbb{R}^{\mathbb{F}_{3}}$.

Proposition 9.8. Let $r \geq 2$ and let $i \in[r]$. Then

$$
\lim _{\epsilon \rightarrow 0^{+}} \operatorname{diam}\left(\mathcal{S}_{\epsilon}^{i}\right)=0 .
$$

Topologically, we have seen that $\widehat{M}^{1}\left(\mathcal{R}_{r}\right)$ is homeomorphic to a simplex with its vertices removed. Proposition 9.8 shows that geometrically $\widehat{\mathcal{M}}^{1}\left(\mathcal{R}_{r}\right)$ is similar to an ideal hyperbolic simplex, with cross-sections whose diameter shrinks to zero as we move toward an ideal vertex.

Given distinct $i, j \in[r]$ and $\epsilon>0$, we let $\ell_{i, j}(\epsilon)$ denote the length function in $\mathcal{M}_{\{i, j\}}^{1}$ where $\left(\ell_{i, j}(\epsilon)\right)^{i}=\epsilon$. As a result, we get that

$$
\left(\ell_{i, j}(\epsilon)\right)^{j}=\log \left(\frac{\exp (-\epsilon)+3}{\exp (-\epsilon)-1}\right)
$$

by Lemma 7.7 .

We begin with a technical lemma that bounds the length of a path in $\mathcal{S}_{\epsilon}^{i}$ to such a point.

Lemma 9.9. Let $r \geq 2$. There is a constant $D$ with the following property. Let $0<\epsilon<$ $\log (2)$ and let $i \in[r]$. Suppose $\ell \in \mathcal{S}_{\epsilon}^{i}$ and that $j \in[r]-\{i\}$ is such that $\ell^{j}=\min \left\{\ell^{k} \mid\right.$ $k \in[r]-\{i\}\}$. Then

$$
d_{\mathfrak{h}, \mathcal{R}_{r}}\left(\ell, \ell_{i, j}(\epsilon)\right) \leq \frac{D}{-\log (\exp (\epsilon)-1)}
$$

Proof. Using the notation of the lemma, we consider the path $\ell_{t}:[0, \infty) \rightarrow \mathcal{S}_{\epsilon}^{i}$ defined by

$$
\ell_{t}^{k}=\ell^{k}+t, \quad k \neq i, j ; \ell_{t}^{i}=\epsilon .
$$

Note that $\ell_{t}^{j}$ is not specified; its value is determined by the constraint that $\mathfrak{h}_{r}\left(\ell_{t}\right)=1$. Notice that $\ell_{t}$ extends to a path $\ell_{t}:[0, \infty] \rightarrow \widehat{\mathcal{M}}^{1}\left(\mathcal{R}_{r}\right)$ and $\ell_{\infty}=\ell_{i, j}(\epsilon) \in \mathcal{M}_{\{i, j\}}^{1}$. We 
observe that since the length of $\ell_{t}^{k}$ is increasing for $k \neq i, j$ and as $\ell_{t}^{i}$ is constant, we have $\ell_{t}^{j} \leq \ell^{j}$ for all $t$.

Given a subset $S \subseteq[r]$, let $|S|_{i}=|S|-1$ if $i \in S$ and let $|S|_{i}=|S|$ otherwise. With this definition, for a subset $S \subseteq[r]-\{j\}$ we have that $\ell_{t}^{S}=\ell^{S}+|S|_{i} t$. Therefore, using the definition of $X_{j}\left(\ell_{t}\right)$ from (7.6) and $Y_{j}\left(\ell_{t}\right)$ from (7.7), we find that

$$
\begin{aligned}
X_{j}\left(\ell_{t}\right) & =\sum_{S \subseteq[r]-\{j\}}(1-2|S|) \exp \left(-\ell^{S}-|S|_{i} t\right), \\
Y_{j}\left(\ell_{t}\right) & =\sum_{S \subseteq[r]-\{j\}}(1+2|S|) \exp \left(-\ell^{S}-|S|_{i} t\right) .
\end{aligned}
$$

Let $p(t)=\log \left(Y_{j}\left(\ell_{t}\right)\right)$ and $q(t)=-\log \left(X_{j}\left(\ell_{t}\right)\right)$, and so $\ell^{j}(t)=p(t)+q(t)$ by (7.9). The next two claims establish bounds on the second derivatives of $p(t)$ and $q(t)$.

Claim 9.10. There is a constant $C_{1}$ such that $|\ddot{p}(t)| \leq C_{1} \exp (-t)$.

Proof of Claim 9.10. Using that fact that $1<Y_{j}\left(\ell_{t}\right)$ from (7.12), we have

$$
|\ddot{p}(t)|=\left|\frac{\ddot{Y}_{j}\left(\ell_{t}\right) Y_{j}\left(\ell_{t}\right)-\dot{Y}_{j}\left(\ell_{t}\right)^{2}}{Y_{j}\left(\ell_{t}\right)^{2}}\right| \leq\left|\frac{\ddot{Y}_{j}\left(\ell_{t}\right)}{Y_{j}\left(\ell_{t}\right)}\right|+\left|\frac{\dot{Y}_{j}\left(\ell_{t}\right)}{Y_{j}\left(\ell_{t}\right)}\right|^{2} \leq\left|\ddot{Y}_{j}\left(\ell_{t}\right)\right|+\left|\dot{Y}_{j}\left(\ell_{t}\right)\right|^{2} .
$$

The summands in $\left|\ddot{Y}_{j}\left(\ell_{t}\right)\right|$ have the form

$$
|S|_{i}^{2}(1+2|S|) \exp \left(-\ell^{S}-|S|_{i} t\right)
$$

The summands in $\left|\dot{Y}_{j}\left(\ell_{t}\right)\right|^{2}$ have the form

$$
|S|_{i}\left|S^{\prime}\right|_{i}(1+2|S|)\left(1+2\left|S^{\prime}\right|\right) \exp \left(-\ell^{S}-\ell^{S^{\prime}}-\left(|S|_{i}+\left|S^{\prime}\right|_{i}\right) t\right) .
$$

Each non-zero term in (9.6) and (9.7) has the form $A \exp (-B-C t)$ where $A, B \geq 0$ and $C \geq 1$. Hence each term is bounded by $A \exp (-t)$ for some $A \geq 0$. The existence of $C_{1}$ is now clear.

Claim 9.11. There is a constant $C_{2}$ such that $|\ddot{q}(t)| \leq C_{2} \exp (-t)$.

Proof of Claim 9.11. Using the facts that $1<Y_{j}\left(\ell_{t}\right)$ from (7.12) and $\exp \left(-\ell_{t}^{j}\right) Y_{j}\left(\ell_{t}\right)=$ $X_{j}\left(\ell_{t}\right)$ from (7.8), we find that $\exp \left(-\ell_{t}^{j}\right) \leq \exp \left(-\ell_{t}^{j}\right) Y_{j}\left(\ell_{t}\right)=X_{j}\left(\ell_{t}\right)$. Hence

$$
\begin{aligned}
|\ddot{q}(t)| & =\left|\frac{\ddot{X}_{j}\left(\ell_{t}^{j}\right) X_{j}\left(\ell_{t}\right)-\dot{X}_{j}\left(\ell_{t}\right)^{2}}{X_{j}\left(\ell_{t}\right)^{2}}\right| \leq\left|\frac{\ddot{X}_{j}\left(\ell_{t}\right)}{X_{j}\left(\ell_{t}\right)}\right|+\left|\frac{\dot{X}_{j}\left(\ell_{t}\right)}{X_{j}\left(\ell_{t}\right)}\right|^{2} \\
& \leq \exp \left(\ell_{t}^{j}\right)\left|\ddot{X}_{j}\left(\ell_{t}\right)\right|+\exp \left(2 \ell_{t}^{j}\right)\left|\dot{X}_{j}\left(\ell_{t}\right)\right|^{2} .
\end{aligned}
$$

The summands in $\exp \left(\ell_{t}^{j}\right)\left|\ddot{X}_{j}\left(\ell_{t}\right)\right|$ have the form

$$
|S|_{i}^{2}(1+2|S|) \exp \left(\ell_{t}^{j}-\ell^{S}-|S|_{i} t\right) .
$$

The summands in $\exp \left(2 \ell_{t}^{j}\right)\left|\dot{X}_{j}\left(\ell_{t}\right)\right|^{2}$ have the form

$$
|S|_{i}\left|S^{\prime}\right|_{i}(1+2|S|)\left(1+2\left|S^{\prime}\right|\right) \exp \left(2 \ell_{t}^{j}-\ell^{S}-\ell^{S^{\prime}}-\left(|S|_{i}+\left|S^{\prime}\right|_{i}\right) t\right) .
$$


As $\ell_{t}^{j} \leq \min \left\{\ell^{k} \mid k \in[r]-\{i\}\right\}$, we find that $\ell_{t}^{j}-\ell^{S} \leq 0$ for all $S \subseteq[r]-\{j\}$ when $|S|_{i} \neq 0$. Hence, as above, each non-zero term in (9.8) and (9.9) has the form $A \exp (-B-$ $C t$ ) where $A, B \geq 0$ and $C \geq 1$. The existence of $C_{2}$ is now clear.

We can now bound the entropy norm of $\left(\ell_{t}, \dot{\ell}_{t}\right)$ using Proposition 4.14. As $\ddot{\ell}_{t}^{k}=0$ for $k \neq j$ and $\ell_{t}^{k} \partial_{k} \bar{F}_{r}\left(\ell_{t}\right)=\ell_{t}^{k} \exp \left(-\ell_{t}^{k}\right) Y_{k}\left(\ell_{t}\right)>0$ for all $k$ from (7.10), we find that

$$
\left\|\left(\ell_{t}, \dot{\ell}_{t}\right)\right\|_{\mathfrak{h}, \mathcal{R}_{r}}^{2}=\frac{\left\langle\ddot{\ell}_{t}, \nabla \bar{F}_{r}\left(\ell_{t}\right)\right\rangle}{\left\langle\ell_{t}, \nabla \bar{F}_{r}\left(\ell_{t}\right)\right\rangle} \leq \frac{\ddot{\ell}_{t}^{j} \partial_{j} \bar{F}_{r}\left(\ell_{t}\right)}{\ell_{t}^{j} \partial_{j} \bar{F}_{r}\left(\ell_{t}\right)}=\frac{\ddot{\ell}_{t}^{j}}{\ell_{t}^{j}} \leq \frac{\ddot{\ell}_{t}^{j}}{\ell^{j}} .
$$

Thus we can bound the length of the path $\ell_{t}$ by

$$
\begin{aligned}
\mathcal{L}_{\mathfrak{h}, \mathcal{R}_{r}}\left(\ell_{t} \mid[0, \infty)\right) & =\int_{0}^{\infty}\left\|\left(\ell_{t}, \dot{\ell}_{t}\right)\right\|_{\mathfrak{h}, \mathcal{R}_{r}} d t \leq \frac{1}{\ell^{j}} \int_{0}^{\infty} \sqrt{\ddot{\ell}_{t}^{j}} d t \\
& \leq \frac{\sqrt{C_{1}+C_{2}}}{\ell^{j}} \int_{0}^{\infty} \exp (-t / 2) d t=\frac{2 \sqrt{C_{1}+C_{2}}}{\ell^{j}} .
\end{aligned}
$$

As $\ell_{j} \geq-\log (\exp (\epsilon)-1)(9.2)$, setting $D=2 \sqrt{C_{1}+C_{2}}$ we complete the proof of the lemma.

Proof of Proposition 9.8. Let $r \geq 2$ and let $D$ be the constant from Lemma 9.9. Fix $i \in[r]$ and let $\ell_{\epsilon} \in \mathcal{S}_{\epsilon}^{i}$, defined by $\ell_{\epsilon}^{i}=\epsilon$ and such that all other $\ell_{\epsilon}^{j}$ are equal. By Lemma 9.9, we have that

$$
d_{\mathfrak{h}, \mathcal{R}_{r}}\left(\ell_{\epsilon}, \ell_{i, j}(\epsilon)\right) \leq \frac{D}{-\log (\exp (\epsilon)-1)}
$$

for all $j \in[r]-\{i\}$. In particular, the set $\left\{\ell_{i, j}(\epsilon) \mid j \in[r]-\{i\}\right\}$ has diameter at most $2 D /(-\log (\exp (\epsilon)-1))$.

Again, by Lemma 9.9, each $\ell \in \mathcal{S}_{\epsilon}^{i}$ has distance at most $D /(-\log (\exp (\epsilon)-1))$ from some point in $\left\{\ell_{i, j}(\epsilon) \mid j \in[r]-\{i\}\right\}$. Hence

$$
\operatorname{diam}\left(\mathcal{S}_{\epsilon}^{i}\right) \leq \frac{3 D}{-\log (\exp (\epsilon)-1)} .
$$

As $-\log (\exp (\epsilon)-1) \rightarrow \infty$ as $\epsilon \rightarrow 0^{+}$, the proof of the proposition is complete.

10. The moduli space of a graph with a separating edge

The purpose of this section is to introduce tools to analyze the entropy metric on the moduli space of a graph with a separating edge. Throughout this section, let $G=\left(V, E, o, \tau,{ }^{-}\right)$ be a finite connected graph which consists of two disjoint connected subgraphs $G_{1}$, with edges $e_{1}^{1}, \ldots, e_{n_{1}}^{1}$, and $G_{2}$, with edges $e_{1}^{2}, \ldots, e_{n_{2}}^{2}$ connected by an edge $e_{0}$. We assume that both $G_{1}$ and $G_{2}$ have rank at least 2 . We begin our analysis in $\S 10.1$ by showing that there exist paths of finite length limiting to any unit-entropy metric on either $G_{1}$ or $G_{2}$. Using this, in $\$ 10.2$ we construct a space $\widehat{\mathcal{M}}^{1}(G)$ that is similar to the construction of $\widehat{\mathcal{M}}^{1}\left(\mathcal{R}_{r}\right)$ from $\S 9$. The main difference is that in this section, we do not bother to construct the entire completion of $\left(\mathcal{M}^{1}(G), d_{\mathfrak{h}, G}\right)$, rather we just add the points that correspond to a length function of entropy 1 on $G_{1} \cup e_{0}$ or $G_{2} \cup e_{0}$ or $G_{1} \cup G_{2}$. This is sufficient for our purposes. Proposition 10.6 is the culmination of this analysis where we show that there is 
a map from $\widehat{\mathcal{M}}^{1}(G)$ to the completion of $\left(\mathcal{M}^{1}(G), d_{\mathfrak{h}, G}\right)$ that collapses these newly added length functions to a single point.

10.1. Finite-length paths in $\mathcal{M}^{1}(G)$. We seek to show that there is a finite-length path in $\left(\mathcal{M}^{1}(G), d_{\mathfrak{h}, G}\right)$ that limits onto an arbitrary unit-entropy metric on either $G_{1}$ or $G_{2}$. This calculation appears in Proposition 10.2. Given a length function $\ell \in \mathcal{M}(G)$ we denote $\ell^{0}=\ell\left(e_{0}\right), \ell^{1}=\left(\ell\left(e_{1}^{1}\right), \ldots, \ell\left(e_{n_{1}}^{1}\right)\right)$ and $\ell^{2}=\left(\ell\left(e_{1}^{2}\right), \ldots, \ell\left(e_{n_{2}}^{2}\right)\right)$.

Given a simplex $\Delta \in C_{G}$ and an edge $e \in E$ of $G$, we recall that $\Delta(e)$ denotes the number of times $e$ or $\bar{e}$ appears as a vertex in a simple cycle contained in $\Delta$. By the construction of $C_{G}$ we have that $\Delta(e) \in\{0,1,2\}$ for any edge. Further, $\Delta\left(e_{0}\right) \in\{0,2\}$ as $e_{0}$ is separating.

Analogous to the functions $Y_{i}$ defined in $\$ 7.2$ that allowed us to isolate the variable $\ell^{i}$ for the $r$-rose, we define a function $Y: \mathcal{M}(G) \rightarrow \mathbb{R}$ by

$$
Y(\ell)=-\sum_{\substack{\Delta \in C_{G} \\ \Delta\left(e_{0}\right)=2}}(-1)^{|\Delta|} \exp \left(-\ell(\Delta)+2 \ell^{0}\right) .
$$

Notice that this function is constant with respect to $\ell^{0}$ as we may write

$$
\ell(\Delta)=\sum_{e \in E_{+}} \Delta(e) \ell(e)
$$

Hence if $\Delta\left(e_{0}\right)=2$, then $\ell(\Delta)-2 \ell^{0}$ has no dependence on $\ell^{0}$. Also we remark that the function $Y: \mathcal{M}(G) \rightarrow \mathbb{R}$ has a continuous extension to $[0, \infty]^{\left|E_{+}\right|}$and is bounded on $[0, \infty]^{\left|E_{+}\right|}$. With this notation we have the following expression for $F_{G}$.

Lemma 10.1. Let $\ell \in \mathcal{M}(G)$. Then $F_{G}(\ell)=F_{G_{1}}\left(\ell^{1}\right) F_{G_{2}}\left(\ell^{2}\right)-\exp \left(-2 \ell^{0}\right) Y(\ell)$.

Proof. Let $\Delta$ be a simplex in $C_{G}$. If $\Delta\left(e_{0}\right)=0$, then $\Delta$ is the join of two (possibly empty) simplices $\Delta_{1} \in C_{G_{1}}$ and $\Delta_{2} \in C_{G_{2}}$. Indeed, if $\Delta=\left\{\gamma_{1}^{1}, \ldots, \gamma_{m_{1}}^{1}, \gamma_{1}^{2}, \ldots, \gamma_{m_{2}}^{2}\right\}$, then $\Delta=\Delta_{1} * \Delta_{2}$ where $\Delta_{i}=\left\{\gamma_{1}^{i}, \ldots, \gamma_{m_{i}}^{i}\right\}$ for $i=1$, 2. We have $|\Delta|=m_{1}+m_{2}=$ $\left|\Delta_{1}\right|+\left|\Delta_{2}\right|$ and thus

$$
(-1)^{|\Delta|} \exp (-\ell(\Delta))=\left((-1)^{\left|\Delta_{1}\right|} \exp \left(-\ell^{1}\left(\Delta_{1}\right)\right)\right)\left((-1)^{\left|\Delta_{2}\right|} \exp \left(-\ell^{2}\left(\Delta_{2}\right)\right)\right) .
$$

Therefore, by Theorem 4.2, we find that

$$
\begin{aligned}
\sum_{\substack{\Delta \in C_{G} \\
\Delta\left(e_{0}\right)=0}}(-1)^{|\Delta|} \exp (-\ell(\Delta))= & \left(\sum_{\Delta_{1} \in C_{G_{1}}}(-1)^{\left|\Delta_{1}\right|} \exp \left(-\ell^{1}\left(\Delta_{1}\right)\right)\right) \\
& \times\left(\sum_{\Delta_{2} \in C_{G_{2}}}(-1)^{\left|\Delta_{2}\right|} \exp \left(-\ell^{2}\left(\Delta_{2}\right)\right)\right) \\
= & F_{G_{1}}\left(\ell^{1}\right) F_{G_{2}}\left(\ell^{2}\right) .
\end{aligned}
$$

If $\Delta\left(e_{0}\right)=2$, then

$$
(-1)^{|\Delta|} \exp (-\ell(\Delta))=\exp \left(-2 \ell^{0}\right)(-1)^{|\Delta|} \exp \left(-\ell(\Delta)+2 \ell^{0}\right)
$$


Hence, by the definition of $Y(\ell)$, we have

$$
\sum_{\substack{\Delta \in C_{G} \\ \Delta\left(e_{0}\right)=2}}(-1)^{|\Delta|} \exp (-\ell(\Delta))=-\exp \left(-2 \ell^{0}\right) Y(\ell) .
$$

As $e_{0}$ is separating, there are no simplices in $C_{G}$ for which $\Delta\left(e_{0}\right)=1$. By Theorem 4.2 again, the lemma follows.

In particular, for $\ell \in \mathcal{M}^{1}(G)$ we have $F_{G}(\ell)=0$ by Lemma 4.4 , and thus

$$
\ell^{0}=\frac{1}{2} \log \left(\frac{Y(\ell)}{F_{G_{1}}\left(\ell^{1}\right) F_{G_{2}}\left(\ell^{2}\right)}\right) .
$$

Using the above expression for $F_{G}$ and $\ell^{0}$, we will give a method of a finite-length path in $G$ for which $\ell^{0} \rightarrow \infty$.

Proposition 10.2. Fix a length function $\ell^{\prime} \in \mathcal{M}^{1}\left(G_{1}\right)$ and let $1<x_{1}, \ldots, x_{n_{1}}<\infty$ be such that $\ell^{\prime}\left(e_{i}^{1}\right)=\log \left(x_{i}\right)$ for $1 \leq i \leq n_{1}$. Let $\ell \in \mathcal{M}^{1}(G)$ be such that $\ell\left(e_{i}^{1}\right)=\log \left(x_{i}+1\right)$ for $1 \leq i \leq n_{1}$ and let $\ell_{t}:[0,1) \rightarrow \widehat{\mathcal{M}}^{1}(G)$ be the path defined by $\ell_{t}\left(e_{i}^{1}\right)=\log \left(x_{i}+\right.$ $1-t), \ell_{t}^{2}=\ell^{2}$ and

$$
\ell_{t}^{0}=\frac{1}{2} \log \left(\frac{Y\left(\ell_{t}\right)}{F_{G_{1}}\left(\ell_{t}^{1}\right) F_{G_{2}}\left(\ell_{t}^{2}\right)}\right) .
$$

Then $\mathcal{L}_{\mathfrak{h}, G}\left(\ell_{t} \mid[0,1)\right)$ is finite and $\ell_{t}^{0} \rightarrow \infty$ as $t \rightarrow 1^{-}$.

Proof. We will use the notation as in the statement of the proposition. As $\mathfrak{h}_{G_{1}}\left(\ell^{\prime}\right)=1$, we must have that $\ell_{t}^{0} \rightarrow \infty$ at $t \rightarrow 1^{-}$. Indeed, if not then the limiting length function has unit entropy, and with this length function the subgraph $G_{1}$ also has unit entropy.

Notice that since $\ell_{t}(e) \partial_{e} F_{G}\left(\ell_{t}\right)>0$ for all $e \in E_{+}$by Lemma 4.4(3), we have that

$$
\left\langle\ell_{t}, \nabla F_{G}\left(\ell_{t}\right)\right\rangle=\sum_{e \in E_{+}} \ell_{t}(e) \partial_{e} F_{G}\left(\ell_{t}\right) \geq \sum_{i=1}^{n_{1}} \ell_{t}\left(e_{i}^{1}\right) \partial_{e_{i}^{1}} F_{G}\left(\ell_{t}\right) .
$$

By Lemma 10.1, we compute that

$$
\partial_{e_{i}^{1}} F_{G}\left(\ell_{t}\right)=F_{G_{2}}\left(\ell_{t}^{2}\right) \partial_{e_{i}^{1}} F_{G_{1}}\left(\ell_{t}^{1}\right)-\exp \left(-2 \ell_{t}^{0}\right) \partial_{e_{i}^{1}} Y\left(\ell_{t}\right) .
$$

Thus since $\partial_{e_{i}^{1}} Y(\ell)$ is bounded on $\mathcal{M}(G)$, we see that $\left\langle\ell_{t}, \nabla F_{G}\left(\ell_{t}\right)\right\rangle$ has a limit, as $t \rightarrow 1^{-}$, that is bounded below by $F_{G_{2}}\left(\ell^{2}\right)\left\langle\ell^{\prime}, \nabla F_{G_{1}}\left(\ell^{\prime}\right)\right\rangle$, which is positive by Lemma 4.4(3) and Lemma 4.9. (We will see in Lemma 10.4(2) that the limit is in fact exactly equal to $F_{G_{2}}\left(\ell^{2}\right)\left\langle\ell^{\prime}, \nabla F_{G_{1}}\left(\ell^{\prime}\right)\right\rangle$.) Hence there is an $\epsilon>0$ such that

$$
\left\langle\ell_{t}, \nabla F_{G}\left(\ell_{t}\right)\right\rangle \geq \epsilon
$$

for all $t \in[0,1)$.

We compute that $\ddot{\ell}_{t}\left(e_{i}^{1}\right)=(-1) /\left(x_{i}+1-t\right)<0$, hence $\ddot{\ell}_{t}\left(e_{i}^{1}\right) \partial_{e_{i}^{1}} F_{G}\left(\ell_{t}\right)<0$ for all $0<t<1$ and $1 \leq i \leq n_{1}$. Clearly $\ddot{\ell}_{t}\left(e_{i}^{2}\right) \partial_{e_{i}^{2}} F_{G}\left(\ell_{t}\right)=0$ for all $1 \leq i \leq n_{2}$ and $0<t<1$. 
Thus

$$
\left\langle\ddot{\ell}_{t}, \nabla F_{G}\left(\ell_{t}\right)\right\rangle \leq \ddot{\ell}_{t}^{0} \partial_{e_{0}} F_{G}\left(\ell_{t}\right) .
$$

To deal with this term, we need the following claim.

CLAIM 10.3. There are polynomials $p, q \in \mathbb{R}[t]$ where $p(t), q(t) \neq 0$ for $t \in[0,1]$ such that

$$
\exp \left(-2 \ell_{t}^{0}\right)=\frac{(1-t) p(t)}{q(t)} .
$$

Proof of Claim 10.3. As $F_{G}\left(\ell_{t}\right)=0$, we have that

$$
\exp \left(-2 \ell_{t}^{0}\right)=\frac{F_{G_{1}}\left(\ell_{t}^{1}\right) F_{G_{2}}\left(\ell_{t}^{2}\right)}{Y\left(\ell_{t}\right)} .
$$

Let $\ell\left(E_{i}\right)=\sum_{j=1}^{n_{i}} \ell\left(e_{j}^{i}\right)$ for $i=1,2$. Notice that we can write $F_{G_{1}}\left(\ell_{t}^{1}\right)$ as

$$
F_{G_{1}}\left(\ell_{t}^{1}\right)=\sum_{\Delta \in C_{G_{1}}}(-1)^{|\Delta|} \prod_{i=1}^{n_{1}}\left(x_{i}+1-t\right)^{-\Delta\left(e_{i}^{1}\right)} .
$$

Hence $\exp \left(2 \ell_{t}\left(E_{1}\right)\right) F_{G_{1}}\left(\ell_{t}^{1}\right)$ is a polynomial in $t$ with real coefficients. Also, we observe that $\exp \left(2 \ell_{t}\left(E_{2}\right)\right) F_{G_{2}}\left(\ell_{t}^{2}\right)$ is a non-zero constant with respect to $t$. By the definition of $\ell_{t}$ we have that $F_{G_{1}}\left(\ell_{1}^{1}\right)=0$. Hence we can write

$$
\exp \left(2 \ell_{t}\left(E_{1}\right)+2 \ell_{t}\left(E_{2}\right)\right) F_{G_{1}}\left(\ell_{t}^{1}\right) F_{G_{2}}\left(\ell_{t}^{2}\right)=(1-t) p(t)
$$

where $p(t) \in \mathbb{R}[t]$. As the left-hand side of this equation does not vanish for $t \in[0,1)$ by Lemma 4.9 , we see that $p(t) \neq 0$ for $t \in[0,1)$. To show that $p(1) \neq 0$, we see that

$$
\begin{aligned}
p(1) & =\left.\frac{d}{d t}\left(\exp \left(2 \ell_{t}\left(E_{1}\right)+2 \ell_{t}\left(E_{2}\right)\right) F_{G_{1}}\left(\ell_{t}^{1}\right) F_{G_{2}}\left(\ell_{t}^{2}\right)\right)\right|_{t=1} \\
& =\exp \left(2 \ell_{1}\left(E_{1}\right)+2 \ell_{1}\left(E_{2}\right)\right) F_{G_{2}}\left(\ell_{1}^{2}\right)\left\langle\dot{\ell}_{1}^{1}, \nabla F_{G_{1}}\left(\ell_{1}^{1}\right)\right\rangle .
\end{aligned}
$$

As $\ell_{1}^{1} \in \mathcal{M}^{1}\left(G_{1}\right)$, we have that $\nabla F_{G_{1}}\left(\ell_{1}^{1}\right)$ is non-zero and parallel to $\nabla \mathfrak{h}_{G_{1}}\left(\ell_{1}^{1}\right)$ by Lemma 4.4. Since $\mathfrak{h}_{G_{1}}\left(\ell_{t}^{1}\right)$ is increasing with respect to $t$ as every edge length is decreasing (past $t=1$ as well), we have that $\left\langle\dot{\ell}_{1}^{1}, \nabla \mathfrak{h}_{G_{1}}\left(\ell_{1}^{1}\right)\right\rangle \neq 0$. Hence $p(1) \neq 0$ as well.

In a similar way, we observe that we can write

$$
\exp \left(2 \ell_{t}\left(E_{1}\right)+2 \ell_{t}\left(E_{2}\right)\right) Y\left(\ell_{t}\right)=q(t)
$$

for some $q(t) \in \mathbb{R}[t]$. As $Y\left(\ell_{t}\right)=\exp \left(2 \ell^{0}\right) F_{G_{1}}\left(\ell_{t}^{1}\right) F_{G_{2}}\left(\ell_{t}^{2}\right)$ by Lemma 10.1 , we see that $Y\left(\ell_{t}\right) \neq 0$ for $t \in[0,1)$ by Lemma 4.9 and hence $q(t) \neq 0$ for $t \in[0,1)$ as well. As $t \rightarrow$ $1^{-}$, we have that $\ell_{t}^{0} \rightarrow \infty$ and thus $(1-t) p(t) / q(t) \rightarrow 0$ as $t \rightarrow 1^{-}$. As $p(1) \neq 0$, we must have that $q(1) \neq 0$ as well.

By Claim 10.3, we compute that

$$
\ddot{\ell}_{t}^{0}=\frac{1}{2}\left(\frac{1}{(1-t)^{2}}-\frac{\ddot{p}(t) p(t)+(\dot{p}(t))^{2}}{(p(t))^{2}}+\frac{\ddot{q}(t) q(t)+(\dot{q}(t))^{2}}{(q(t))^{2}}\right) .
$$


Using Lemma 10.1 and Claim 10.3, we find that

$$
\partial_{e_{0}} F_{G}(\ell)=2 \exp \left(-2 \ell^{0}\right) Y(\ell)=2 Y(\ell) \frac{(1-t) p(t)}{q(t)} .
$$

Hence we see that there exists a constant $C>0$ such that

$$
\ddot{\ell}_{t}^{0} \partial_{e_{0}} F_{G}\left(\ell_{t}\right) \leq \frac{C}{1-t} .
$$

Therefore, by combining Proposition 4.6 with (10.5), (10.6) and (10.7), we have that

$$
\left\|\left(\ell_{t}, \dot{\ell}_{t}\right)\right\|_{\mathfrak{h}, G}^{2}=\frac{\left\langle\ddot{\ell}_{t}, \nabla F_{G}\left(\ell_{t}\right)\right\rangle}{\left\langle\ell_{t}, \nabla F_{G}\left(\ell_{t}\right)\right\rangle} \leq \frac{\ddot{\ell}_{t}^{0} \partial_{e_{0}} F_{G}\left(\ell_{t}\right)}{\left\langle\ell_{t}, \nabla F_{G}\left(\ell_{t}\right)\right\rangle} \leq \frac{C}{\epsilon(1-t)} .
$$

Hence the length of $\ell_{t}$ is finite.

10.2. The model space $\widehat{\mathcal{M}}^{1}(G)$. The previous example shows that we should expect some points in the completion of $\left(\mathcal{M}^{1}(G), d_{\mathfrak{h}, G}\right)$ to correspond to unit-entropy metrics on $G_{1}$ or $G_{2}$. For the model, we add these points to $\mathcal{M}^{1}(G)$ as well as points that correspond to unit-entropy metrics on $G_{1} \cup G_{2}$. To this end, we set

$$
\begin{aligned}
\mathcal{M}_{1} & =\left\{\ell \in(0, \infty]^{\left|E_{+}\right|} \mid \ell^{1} \in \mathcal{M}\left(G_{1}\right) \text { and } \ell^{2}=\infty \cdot \mathbf{1}\right\}, \\
\mathcal{M}_{2} & =\left\{\ell \in(0, \infty]^{\left|E_{+}\right|} \mid \ell^{1}=\infty \cdot \mathbf{1} \text { and } \ell^{2} \in \mathcal{M}\left(G_{2}\right)\right\}, \\
\mathcal{M}_{1,2} & =\left\{\ell \in(0, \infty]^{\left|E_{+}\right|} \mid \ell^{0}=\infty, \ell^{1} \in \mathcal{M}\left(G_{1}\right) \text { and } \ell^{2} \in \mathcal{M}\left(G_{2}\right)\right\} .
\end{aligned}
$$

We consider their union $\widehat{\mathcal{M}}(G)=\mathcal{M}(G) \cup \mathcal{M}_{1} \cup \mathcal{M}_{2} \cup \mathcal{M}_{1,2}$ as a subset of $(0, \infty]^{\left|E_{+}\right|}$, endowed with the subspace topology as in $\S 9$. There are obvious embeddings $\varepsilon_{i}: \mathcal{M}\left(G_{i}\right) \rightarrow \mathcal{M}_{i}$ for $i=1,2$ where we set $\varepsilon_{i}(\ell)^{0}=\infty$, and an obvious embedding $\varepsilon_{1,2}: \mathcal{M}\left(G_{1}\right) \times \mathcal{M}\left(G_{2}\right) \rightarrow \mathcal{M}_{1,2}$ as well. Next, we define

$$
\begin{aligned}
\mathcal{M}_{1}^{1} & =\left\{\ell \in \mathcal{M}_{1} \mid \mathfrak{h}_{G_{1}}\left(\ell^{1}\right)=1\right\}, \\
\mathcal{M}_{2}^{1} & =\left\{\ell \in \mathcal{M}_{2} \mid \mathfrak{h}_{G_{2}}\left(\ell^{2}\right)=1\right\}, \\
\mathcal{M}_{1,2}^{1} & =\left\{\ell \in \mathcal{M}_{1,2} \mid \max \left\{\mathfrak{h}_{G_{1}}\left(\ell^{1}\right), \mathfrak{h}_{G_{2}}\left(\ell^{2}\right)\right\}=1\right\} .
\end{aligned}
$$

Our model space is the union of these sets. Specifically, we define

$$
\widehat{\mathcal{M}}^{1}(G)=\mathcal{M}^{1}(G) \cup \mathcal{M}_{1}^{1} \cup \mathcal{M}_{2}^{1} \cup \mathcal{M}_{1,2}^{1} .
$$

Using (4.2), we see that each partial derivative of $F_{G}$ extends to a bounded continuous function on $\widehat{\mathcal{M}}(G)$. The naturality of these extensions is the same as in Lemma 9.3. Even more, the inner product $\left\langle\ell, \nabla F_{G}(\ell)\right\rangle$ has a continuous extension as was the case in Lemma 9.4.

LEMMA 10.4. The function $\ell \mapsto\left\langle\ell, \nabla F_{G}(\ell)\right\rangle$ has a continuous extension to $\widehat{\mathcal{M}}(G)$. This extension is such that the following statements hold.

(1) If $i \in\{1,2\}$ and $\ell \in \mathcal{M}_{i}$, then

$$
\left\langle\ell, \nabla F_{G}(\ell)\right\rangle=\left\langle\ell^{i}, \nabla F_{G_{i}}\left(\ell^{i}\right)\right\rangle .
$$


(2) If $\ell \in \mathcal{M}_{1,2}$, then

$$
\left\langle\ell, \nabla F_{G}(\ell)\right\rangle=F_{G_{2}}\left(\ell^{2}\right)\left\langle\ell^{1}, \nabla F_{G_{1}}\left(\ell^{1}\right)\right\rangle+F_{G_{1}}\left(\ell^{1}\right)\left\langle\ell^{2}, \nabla F_{G_{2}}\left(\ell^{2}\right)\right\rangle .
$$

(3) For all $\ell \in \widehat{\mathcal{M}}(G)$, we have $\left\langle\ell, \nabla F_{G}(\ell)\right\rangle \geq 0$ with equality if and only if $\mathfrak{h}_{G_{1}}\left(\ell^{1}\right)=$ $\mathfrak{h}_{G_{2}}\left(\ell^{2}\right)=1$.

Proof. From Lemma 4.4(3) and the expression for $\partial_{e} F_{G}(\ell)$ in (4.2), we see that there is a constant $A>0$ such that $0<\partial_{e} F_{G}(\ell) \leq A \exp (-\ell(e))$ for any edge $e \in E_{+}$. The existence of the continuous extension now follows for the same reason as for Lemma 9.4.

If $\ell^{1}=\infty \cdot \mathbf{1}$, then $Y(\ell)=0$. Indeed, this follows as every simple cycle in $G$ that contains $e_{0}$ must also contain an edge in $G_{1}$ as $e_{0}$ is separating. Likewise, if $\ell^{2}=\infty \cdot \mathbf{1}$, then $Y(\ell)=0$ as well. Hence $\partial_{e_{0}} F_{G}(\ell)=2 \exp \left(-2 \ell^{0}\right) Y(\ell)=0$ for $\ell \in \mathcal{M}_{i}$ when $i=$ 1, 2. This, together with the paragraph above, shows (1).

Using Lemma 10.1, we compute the following expression for $\left\langle\ell, \nabla F_{G}(\ell)\right\rangle$ :

$$
\begin{aligned}
\left\langle\ell, \nabla F_{G}(\ell)\right\rangle= & F_{G_{2}}\left(\ell^{2}\right)\left\langle\ell^{1}, \nabla F_{G_{1}}\left(\ell^{1}\right)\right\rangle+F_{G_{1}}\left(\ell^{1}\right)\left\langle\ell^{2}, \nabla F_{G_{2}}\left(\ell^{2}\right)\right\rangle \\
& -\exp \left(-2 \ell^{0}\right)\left(\langle\hat{\ell}, \nabla Y(\hat{\ell})\rangle-2 \ell^{0} Y(\hat{\ell})\right)
\end{aligned}
$$

From this (2) is apparent.

As $\left\langle\ell, \nabla F_{G}(\ell)\right\rangle>0$ for all $\ell \in \mathcal{M}^{1}(G)$ by Lemma $4.4(3)$, the extension is clearly non-negative. Statement (3) now follows from (1) and (2) as $\left\langle\ell, \nabla F_{G_{i}}(\ell)\right\rangle>0$ for any $\ell \in \mathcal{M}^{1}\left(G_{i}\right)$ again by Lemma 4.4(3) and $F_{G_{i}}(\ell)>0$ for any $\ell \in \mathcal{M}^{1}\left(G_{i}\right)$ if $\mathfrak{h}\left(G_{i}\right)(\ell)<1$ by Lemma 4.9 .

Next we partition $\widehat{\mathcal{M}}^{1}(G)$ into two subsets that we call the singular points and regular points, respectively:

$$
\begin{aligned}
\widehat{\mathcal{M}}^{1}(G)_{\text {sing }} & =\left\{\ell \in \widehat{\mathcal{M}}^{1}(G) \mid \mathfrak{h}_{G_{1}}\left(\ell^{1}\right)=\mathfrak{h}_{G_{2}}\left(\ell^{2}\right)=1\right\}, \\
\widehat{\mathcal{M}}^{1}(G)_{\text {reg }} & =\widehat{\mathcal{M}}^{1}(G)-\widehat{\mathcal{M}}^{1}(G)_{\text {sing }} .
\end{aligned}
$$

Notice that $\widehat{\mathcal{M}}^{1}(G)_{\text {sing }}$ is a subset of $\mathcal{M}_{1,2}^{1}$.

As in $\$ 9.1$, we also define the tangent bundle $T \widehat{\mathcal{M}}^{1}(G)$ to be the subspace of $(\ell, \mathbf{v}) \in \widehat{\mathcal{M}}^{1}(G) \times \mathbb{R}^{\left|E_{+}\right|}$where $\left\langle\mathbf{v}, \nabla F_{G}(\ell)\right\rangle=0$. There are obvious embeddings $T \varepsilon_{i}: T \mathcal{M}^{1}\left(G_{i}\right) \rightarrow T \widehat{\mathcal{M}}^{1}(G)$ for $i=1,2$ defined using $\varepsilon_{i}: \mathcal{M}^{1}\left(G_{i}\right) \rightarrow \mathcal{M}_{i}$ as in \$9.1. We define $T \widehat{\mathcal{M}}_{\text {reg }}^{1}(G)$ to be the subset of $(\ell, \mathbf{v}) \in T \widehat{\mathcal{M}}^{1}(G)$ where $\ell \in \widehat{\mathcal{M}}^{1}(G)_{\text {reg }}$. Proposition 4.6 together with Lemma 10.4 implies the following proposition.

Proposition 10.5. The entropy norm $\|\cdot\|_{\mathfrak{h}, G}: T \mathcal{M}^{1}(G) \rightarrow \mathbb{R}$ extends to a continuous semi-norm $\|\cdot\|_{\mathfrak{h}, G}: T \widehat{\mathcal{M}}^{1}(G)_{\text {reg }} \rightarrow \mathbb{R}$. Moreover, the embedding maps $T \varepsilon_{i}: T \mathcal{M}^{1}\left(G_{i}\right) \rightarrow$ $T \widehat{\mathcal{M}}^{1}(G)$ are norm-preserving.

As in $\$ 9.1$, we have the following proposition that shows us that there is a map from $\widehat{\mathcal{M}}^{1}(G)$ to the completion of $\mathcal{M}^{1}(G)$ with respect to the entropy metric. 
Proposition 10.6. The following statements hold.

(1) The entropy norm defines a pseudo-metric $d_{\mathfrak{h}, G}$ on $\widehat{\mathcal{M}}^{1}(G)$.

(2) We have $\operatorname{diam}\left(\mathcal{M}_{1}^{1} \cup \mathcal{M}_{2}^{1} \cup \mathcal{M}_{1,2}^{1}\right)=0$.

(3) The inclusion $\left(\mathcal{M}^{1}(G), d_{\mathfrak{h}, G}\right) \rightarrow\left(\widehat{\mathcal{M}}^{1}(G), d_{\mathfrak{h}, G}\right)$ is an isometric embedding.

Proof. As in Proposition 9.6, we need to show that for any $\ell, \ell^{\prime} \in \widehat{\mathcal{M}}^{1}(G)$ there is a path $\ell_{t}:[0,1] \rightarrow \widehat{\mathcal{M}}(G)$ with $\ell_{0}=\ell$ and $\ell_{1}=\ell^{\prime}$ that has finite length.

To this end, fix a point $\ell \in \mathcal{M}^{1}(G)$. There are several cases depending on whether $\ell^{\prime} \in$ $\mathcal{M}_{1}^{1}, \ell^{\prime} \in \mathcal{M}_{2}^{1}$ or $\ell^{\prime} \in \mathcal{M}_{1,2}^{1}$.

We first deal with the case that $\ell^{\prime} \in \mathcal{M}_{1,2}^{1}$. Without loss of generality we assume that $\mathfrak{h}_{G_{1}}\left(\left(\ell^{\prime}\right)^{1}\right)=1$. In Proposition 10.2 we produced a path $\ell_{t}:[0,1] \rightarrow \widehat{\mathcal{M}}^{1}(G)$ where $\ell_{0} \in$ $\mathcal{M}^{1}(G)$ and $\ell_{1} \in \mathcal{M}_{1,2}$ is such that $\ell_{1}^{1}=\left(\ell^{\prime}\right)^{1}$. We can concatenate the path $\ell_{t}$ with a path $\tilde{\ell}_{t}:[0,1] \rightarrow \mathcal{M}_{1,2}^{1}$ from $\ell_{1}$ to $\ell^{\prime}$ in $\mathcal{M}_{1,2}^{1}$ as follows. We define the path by

$$
\tilde{\ell}_{t}=\left(\infty, \ell_{1}^{1},(1-t) \cdot \ell_{1}^{2}+t \cdot\left(\ell^{\prime}\right)^{2}\right)
$$

and we observe that $\tilde{\ell}_{0}=\ell_{1}$ to $\tilde{\ell}_{1}=\ell^{\prime}$. Note that by the convexity of entropy we have that

$$
\mathfrak{h}_{G_{2}}\left((1-t) \cdot \ell_{1}^{2}+t \cdot\left(\ell^{\prime}\right)^{2}\right) \leq 1
$$

with equality only possible at the endpoints. Hence the interior of the path $\tilde{\ell}_{t}$ lies in $\widehat{\mathcal{M}}^{1}(G)_{\text {reg. }}$. Further,

$$
\left\|\left(\tilde{\ell}_{t}, \dot{\tilde{\ell}}_{t}\right)\right\|_{\mathfrak{h}, G}=0
$$

as the length of edges in $G_{2}$ is changing linearly. Hence there is a path of finite length from a length function in $\mathcal{M}^{1}(G)$ to any length function in $\mathcal{M}_{1,2}^{1}$.

Next, we deal with the case that $\ell^{\prime} \in \mathcal{M}_{1}$; the case of $\ell^{\prime} \in \mathcal{M}_{2}$ is symmetric. We will show that we can connect $\ell^{\prime}$ to a length function in $\mathcal{M}_{1,2}$ with a concatenation of two paths that have finite length-in fact each has length 0 . Let $\ell^{\prime \prime} \in \mathcal{M}\left(G_{2}\right)$ have entropy less than 1 . The paths $\left(\ell_{1}\right)_{t}$ and $\left(\ell_{2}\right)_{t}$ are as follows:

$$
\begin{array}{ll}
\left(\ell_{1}\right)_{t}:[0, \infty] \rightarrow \widehat{\mathcal{M}}^{1}(G) & \left(\ell_{1}\right)_{t}=\left(\left(\ell^{\prime}\right)^{0}+t,\left(\ell^{\prime}\right)^{1}, \infty \cdot \mathbf{1}\right), \\
\left(\ell_{2}\right)_{t}:[0, \infty] \rightarrow \widehat{\mathcal{M}}^{1}(G) & \left(\ell_{2}\right)_{t}=\left(\infty,\left(\ell^{\prime}\right)^{1},\left(\ell^{\prime}\right)^{2}+t \cdot \mathbf{1}\right) .
\end{array}
$$

The concatenation of $\left(\ell_{1}\right)_{t} \mid[0, \infty]$ and $\left(\ell_{2}\right)_{t} \mid[\infty, 0]$ is a path from $\ell^{\prime}$ to $\ell^{\prime \prime}$. We observe that $\left(\ddot{\ell}_{1}\right)_{t}=0$ and $\left(\ddot{\ell}_{2}\right)_{t}=0$ as edge lengths are changing linearly. Also, we observe that the interiors of these paths lie in $\widehat{\mathcal{M}}^{1}(G)_{\text {reg. }}$. Hence $\left\|\left(\left(\ell_{k}\right)_{t},\left(\dot{\ell}_{k}\right)_{t}\right)\right\|_{\mathfrak{h}, G}=0$ for $k=1,2$ showing that the paths have finite-in fact zero-length.

This shows (10.6).

The previous argument shows that for any $\ell \in \mathcal{M}_{1}^{1}$, there is an $\ell^{\prime} \in \mathcal{M}_{1,2}^{1}$ such that $d_{\mathfrak{h}, G}\left(\ell, \ell^{\prime}\right)=0$. Likewise the analogous statement holds for $\ell \in \mathcal{M}_{2}^{1}$. Given, $\ell, \ell^{\prime} \in \mathcal{M}_{1,2}$, we will show that $d_{\mathfrak{h}, G}\left(\ell, \ell^{\prime}\right)=0$, completing the proof of (10.6). There are four cases depending on the entropies of the length functions $\ell^{1}, \ell^{2},\left(\ell^{\prime}\right)^{1}$ and $\left(\ell^{\prime}\right)^{2}$. 
The first case we consider is when $\mathfrak{h}_{G_{1}}\left(\ell^{1}\right)=1$ and $\mathfrak{h}_{G_{2}}\left(\left(\ell^{\prime}\right)^{2}\right)=1$. In this case we consider the concatenation of the two paths $\left(\ell_{1}\right)_{t}$ and $\left(\ell_{2}\right)_{t}$ that are defined as follows:

$$
\begin{array}{ll}
\left(\ell_{1}\right)_{t}:[0,1] \rightarrow \widehat{\mathcal{M}}^{1}(G) & \left(\ell_{1}\right)_{t}=\left(\infty, \ell^{1},(1-t) \cdot \ell^{2}+t \cdot\left(\ell^{\prime}\right)^{2}\right), \\
\left(\ell_{2}\right)_{t}:[0,1] \rightarrow \widehat{\mathcal{M}}^{1}(G) & \left(\ell_{2}\right)_{t}=\left(\infty,(1-t) \cdot \ell^{1}+t \cdot\left(\ell^{\prime}\right)^{1},\left(\ell^{\prime}\right)^{2}\right) .
\end{array}
$$

As above, the interiors of these paths lie in $\widehat{\mathcal{M}}^{1}(G)_{\text {reg }}$ and they have length 0 since the lengths of edges are changing linearly. This completes this case.

The case where $\mathfrak{h}_{G_{2}}\left(\ell^{2}\right)=1$ and $\mathfrak{h}_{G_{1}}\left(\left(\ell^{\prime}\right)^{1}\right)=1$ is similar.

Next we consider the case where $\mathfrak{h}_{G_{1}}\left(\ell^{1}\right)=1$ and $\mathfrak{h}_{G_{1}}\left(\left(\ell^{\prime}\right)^{1}\right)=1$. Fix a length function $\ell^{\prime \prime} \in \mathcal{M}_{1,2}^{1}$ with $\mathfrak{h}_{G_{2}}\left(\left(\ell^{\prime \prime}\right)^{2}\right)=1$. By the first argument, we can connect both $\ell$ and $\ell^{\prime}$ to $\ell^{\prime \prime}$ with paths of length 0 . Concatenating these two paths shows that this case holds as well.

The case where $\mathfrak{h}_{G_{2}}\left(\ell^{2}\right)=1$ and $\mathfrak{h}_{G_{2}}\left(\left(\ell^{\prime}\right)^{2}\right)=1$ is similar.

This completes the proof of (10.6).

We observe that any path in $\widehat{\mathcal{M}}^{1}(G)$ is close to a path in $\mathcal{M}^{1}(G)$. Thus by Proposition 10.5 , we have that the inclusion $\left.\left(\mathcal{M}^{1}(G), d_{\mathfrak{h}, G}\right) \rightarrow \widehat{\mathcal{M}}^{1}(G), d_{\mathfrak{h}, G}\right)$ is an isometric embedding, hence (10.6) holds.

\section{1. $\mathcal{X}^{1}\left(\mathcal{R}_{r}, i d\right)$ has bounded diameter in $\mathcal{X}^{1}\left(\mathbb{F}_{r}\right)$}

In this section we make use of the collapsing phenomena witnessed in the previous section to show that even though $\left(\mathcal{M}^{1}\left(\mathcal{R}_{r}\right), d_{\mathfrak{h}, \mathcal{R}_{r}}\right)$ has infinite diameter (Proposition 7.14), the subspace $\left(\mathcal{X}^{1}\left(\mathcal{R}_{r}, \mathrm{id}\right), d_{\mathfrak{h}}\right) \subset\left(\mathcal{X}^{1}\left(\mathbb{F}_{r}\right), d_{\mathfrak{h}}\right)$ has bounded diameter. The idea is as follows. Using the natural bijection $\mathcal{M}^{1}\left(\mathcal{R}_{r}\right) \leftrightarrow \mathcal{X}^{1}\left(\mathcal{R}_{r}\right.$, id $)$, since $\widehat{\mathcal{M}}^{1}\left(\mathcal{R}_{r}\right)$ is the completion of $\left(\mathcal{M}^{1}\left(\mathcal{R}_{r}\right), d_{\mathfrak{h}, \mathcal{R}_{r}}\right)(\S 9)$ there is a map $\Phi: \widehat{\mathcal{M}}^{1}\left(\mathcal{R}_{r}\right) \rightarrow \widehat{\mathcal{X}}^{1}\left(\mathbb{F}_{r}\right)$ where $\widehat{\mathcal{X}}^{1}\left(\mathbb{F}_{r}\right)$ is the completion of $\left(\mathcal{X}^{1}\left(\mathbb{F}_{r}\right), d_{\mathfrak{h}}\right)$. Indeed, if a sequence $\left(\ell_{n}\right) \subset\left(\mathcal{M}^{1}\left(\mathcal{R}_{r}\right), d_{\mathfrak{h}, \mathcal{R}_{r}}\right)$ is Cauchy, then so is its image under $\Phi$ in $\left(X^{1}\left(\mathbb{F}_{r}\right), d_{\mathfrak{h}}\right)$ as $\Phi$ is 1-Lipschitz. As $\widehat{\mathcal{M}}^{1}\left(\mathcal{R}_{r}\right)$ is homeomorphic to the complement of the vertices of an $(r-1)$-simplex, we can consider $\Phi$ as the map $\Phi: \Delta^{r-1}-V \rightarrow \widehat{x}^{1}\left(\mathbb{F}_{r}\right)$ where $\Delta^{r-1}$ is the standard $(r-1)$-dimensional simplex and $V \subset \Delta^{r-1}$ is the set of vertices. We will show that the map $\Phi$ extends to the vertex set $V$. Since the image $\Phi\left(\Delta^{r-1}\right)$ is compact and contains $\mathcal{X}^{1}\left(\mathcal{R}_{r}\right.$, id $)$, it follows that $\left(\mathcal{X}^{1}\left(\mathcal{R}_{r}, \mathrm{id}\right), d_{\mathfrak{h}}\right)$ has bounded diameter.

This is accomplished by considering $\mathcal{M}^{1}\left(\mathcal{R}_{r}\right)$ as the face of $\mathcal{M}^{1}(G)$ for a particular graph $G$ that has a separating edge and using the tools developed in $\S 10$. Lemma 11.1 establishes that the subset $\bigcup\left\{\mathcal{M}_{S}|1<| S \mid<r-1\right\} \subset \widehat{\mathcal{M}}^{1}\left(\mathcal{R}_{r}\right)$ is collapsed to a single point in the completion of $\left(\mathcal{X}^{1}\left(\mathbb{F}_{r}\right), d_{\mathfrak{h}}\right)$. We recall that $\mathcal{M}_{S}^{1} \subset \widehat{\mathcal{M}}^{1}\left(\mathcal{R}_{r}\right)$ is the subset of unit-entropy length functions on the subrose $\mathcal{R}_{|S|} \subseteq \mathcal{R}_{r}$ utilizing the edges in $S \subseteq[r]$; the length of an edge in $[r]-S$ is $\infty$. The subset $\mathcal{M}_{S}^{1}$ corresponds to an $(|S|-1)$-dimensional face of $\Delta^{r-1}$. Thus Lemma 11.1 shows that the entire $(r-3)$-skeleton of $\Delta^{r-1}$ is collapsed to a point by $\Phi$ in $\widehat{x}^{1}\left(\mathbb{F}_{r}\right)$.

LEMMA 11.1. For $r \geq 4$, $\Phi$ maps the subset $\bigcup\left\{\mathcal{M}_{S}|1<| S \mid<r-1\right\} \subset \widehat{\mathcal{M}}^{1}\left(\mathcal{R}_{r}\right)$ to a single point in $\widehat{x}^{1}\left(\mathbb{F}_{r}\right)$. 


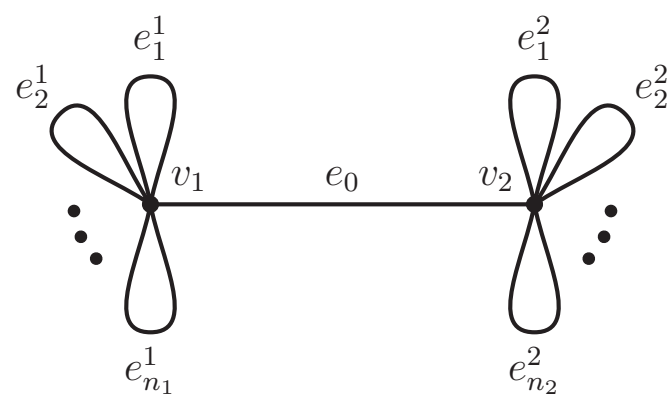

FIGURE 7. The graph $G_{n_{1}, n_{2}}$ : there are $n_{1}$ loop edges attached to $v_{1}$ and $n_{2}$ loop edges attached to $v_{2}$.

Proof. Fix $r \geq 4$ and let $S$ be a subset of $[r]$ with $1<|S|<r-1$. To begin, we claim that the image of $\mathcal{M}_{S}^{1}$ is a single point in $\widehat{x}^{1}\left(\mathbb{F}_{r}\right)$. To this end, we set $n_{1}=|S|$ and $n_{2}=r-|S|$. Notice that $n_{1}, n_{2} \geq 2$. Let $G_{n_{1}, n_{2}}$ be the graph that consists of two vertices $v_{1}$ and $v_{2}$, and edges $e_{0}, e_{1}^{1}, \ldots, e_{n_{1}}^{1}$ and $e_{1}^{2}, \ldots, e_{n_{2}}^{2}$. The edges are attached via the following table.

\begin{tabular}{c|c|c} 
& $o$ & $\tau$ \\
\hline$e_{0}$ & $v_{1}$ & $v_{2}$ \\
\hline$e_{i}^{1}$ & $v_{1}$ & $v_{1}$ \\
\hline$e_{i}^{2}$ & $v_{2}$ & $v_{2}$ \\
\hline
\end{tabular}

See Figure 7. We adopt the notation introduced in $\S 10$ for $G_{n_{1}, n_{2}}$.

Let $c: G_{n_{1}, n_{2}} \rightarrow \mathcal{R}_{r}$ be the map induced by collapsing the edge $e_{0}$ and let $\rho: \mathcal{R}_{r} \rightarrow$ $G_{n_{1}, n_{2}}$ be a map so that $c \circ \rho$ is homotopic to id: $\mathcal{R}_{r} \rightarrow \mathcal{R}_{r}$. Thus

$$
\mathcal{X}^{1}\left(\mathcal{R}_{r}, \text { id }\right) \subset \overline{\mathcal{X}}^{1}\left(G_{n_{1}, n_{2}}, \rho\right)=\left\{\left[\left(G, \rho^{\prime}, \ell\right)\right] \in \overline{\mathcal{X}}\left(G_{n_{1}, n_{2}}, \rho\right) \mid \mathfrak{h}_{G}(\ell)=1\right\} .
$$

Specifically, viewing $\overline{\mathcal{X}}^{1}\left(G_{n_{1}, n_{2}}, \rho\right)$ as a subset of $[0, \infty)^{1+n_{1}+n_{2}}$, we see that $\mathcal{X}^{1}\left(\mathcal{R}_{r}\right.$, id) is the image of the embedding $\varepsilon: \mathcal{M}^{1}\left(\mathcal{R}_{r}\right) \rightarrow[0, \infty)^{1+n_{1}+n_{2}}$ where $\varepsilon(\ell)^{0}=0$.

Moreover, $\varepsilon$ extends to an embedding $\widehat{\mathcal{M}}^{1}\left(\mathcal{R}_{r}\right) \rightarrow[0, \infty]^{1+n_{1}+n_{2}}$ in the same way. Under this embedding, $\varepsilon\left(\mathcal{M}_{S}^{1}\right)$ is the face of $\mathcal{M}_{1}^{1} \subset \widehat{\mathcal{M}}^{1}\left(G_{n_{1}, n_{2}}\right) \subset[0, \infty]^{1+n_{1}+n_{2}}$. Indeed, the set $\mathcal{M}_{1}^{1}$ is homeomorphic to $(0, \infty] \times \mathcal{M}_{S}^{1}$. By Proposition $10.6(2)$, the set $\mathcal{M}_{1}^{1}$ maps to a single point in $\widehat{x}^{1}\left(\mathbb{F}_{r}\right)$. Hence so does $\mathcal{M}_{S}^{1}$, completing the proof of our claim.

As the diameter of the thin part goes to zero as the short edge goes to zero (Proposition 9.8), the point that $\mathcal{M}_{S}^{1}$ is sent to is independent of $S$.

The main result of this section now follows easily.

PROposition 11.2. For $r \geq 4$, the subspace $\left(\mathcal{X}^{1}\left(\mathcal{R}_{r}, i d\right), d_{\mathfrak{h}}\right) \subset\left(\mathcal{X}^{1}\left(\mathbb{F}_{r}\right), d_{\mathfrak{h}}\right)$ has bounded diameter.

Proof. As explained above in the introduction to this section, by Theorem 1.2, there is a map $\Phi: \Delta^{r-1}-V \rightarrow \widehat{x}^{1}\left(\mathbb{F}_{r}\right)$. By Lemma 11.1, the map $\Phi$ extends to $V$ and the entire 
$\Gamma_{r}$

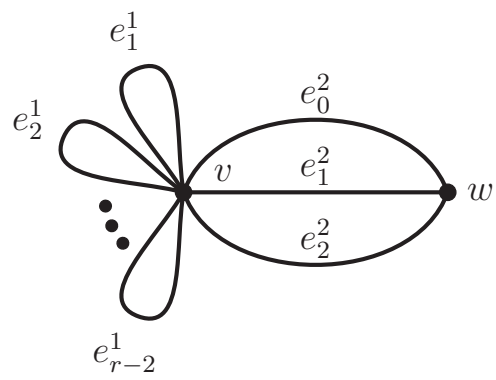

$\widehat{\Gamma}_{r}$

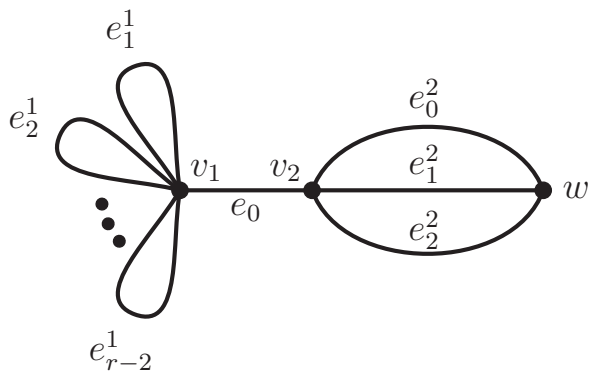

FIGURE 8. The graphs $\Gamma_{r}$ and $\widehat{\Gamma}_{r}$. In $\Gamma_{r}$ there are $r-2$ loop edges attached to $v$ and three edges connecting $v$ to $w$. In $\widehat{\Gamma}_{r}$, there are $r-2$ loop edges attached to $v_{1}$, three edges connecting $v_{2}$ to $w$ and a separating edge connecting $v_{1}$ to $v_{2}$.

$(r-3)$-skeleton of $\Delta^{r-1}$ is mapped to a single point. As $\Delta^{r-1}$ is compact, $\Phi\left(\Delta^{r-1}\right)$ is compact and hence has bounded diameter. Thus $\mathcal{X}^{1}\left(\mathcal{R}_{r}, \mathrm{id}\right) \subset \Phi\left(\Delta^{r-1}\right) \subset \mathcal{X}^{1}(\mathbb{F})$ has bounded diameter too.

\section{Proof of Theorem 1.3}

The goal of this final section is the proof of the last of the main results. Theorem 1.3 states that the Out $\left(\mathbb{F}_{r}\right)$-invariant subcomplex $\left(\mathcal{X}^{1}\left(\mathcal{R}_{r}, \mathrm{id}\right) \cdot \operatorname{Out}\left(\mathbb{F}_{r}\right), d_{\mathfrak{h}}\right)$ has bounded diameter and, moreover, that the action of $\operatorname{Out}\left(\mathbb{F}_{r}\right)$ on the completion of $\left(\mathcal{X}^{1}\left(\mathbb{F}_{r}\right), d_{\mathfrak{h}}\right)$ has a global fixed point. This point is the image of $\bigcup\left\{\mathcal{M}_{S}|1<| S \mid<r-1\right\}$ for any marking of the rose. We show that the image of this point in the completion is independent of the marking. This is done by showing that it is independent for markings that differ by a single simple move-we call such markings Nielsen adjacent. This is accomplished again by making use of a graph with a separating edge and the analysis in $\S 10$. This simple move suffices to connect any two markings and the theorem easily follows.

Proof of Theorem 1.3. Given a marked rose $\left(\mathcal{R}_{r}, \rho\right)$, there is an embedding $\Phi_{\rho}: \mathcal{M}^{1}$ $\left(\mathcal{R}_{r}\right) \rightarrow \mathcal{X}^{1}\left(\mathbb{F}_{r}\right)$ whose image is $\mathcal{X}^{1}\left(\mathcal{R}_{r}, \rho\right)$. As in $\S 11$, this map extends to $\Phi_{\rho}: \widehat{\mathcal{M}}^{1}\left(\mathcal{R}_{r}\right) \rightarrow$ $\widehat{\chi}^{1}\left(\mathbb{F}_{r}\right)$ where $\widehat{\chi}^{1}\left(\mathbb{F}_{r}\right)$ is the completion of $\mathcal{X}^{1}\left(\mathbb{F}_{r}\right)$ with the entropy metric. By Lemma 11.1, $\Phi_{\rho}$ maps $\bigcup\left\{\mathcal{M}_{S}^{1}|1<| S \mid<r-1\right\}$ to a single point in $\widehat{\mathcal{X}}^{1}\left(\mathbb{F}_{r}\right)$. Let $x_{\rho}$ denote this point in $\overline{\mathcal{X}}(\mathbb{F})$.

Given an integer $r>2$, we define a graph $\Gamma_{r}$ that has two vertices $v$ and $w$, and edges $e_{1}^{1}, \ldots, e_{r-2}^{1}$ and $e_{0}^{2}, e_{1}^{2}, e_{3}^{2}$. The edges are attached via the following table.

\begin{tabular}{c|c|c} 
& $o$ & $\tau$ \\
\hline$e_{i}^{1}$ & $v$ & $v$ \\
\hline$e_{i}^{2}$ & $v$ & $w$ \\
\hline
\end{tabular}

See Figure 8. We call such a graph a rose-theta graph. 
Given $i \in\{0,1,2\}$, collapsing the edge $e_{i}^{2}$ induces a map $c_{i}: \Gamma_{r} \rightarrow \mathcal{R}_{r}$. We say two marked roses $\left(\mathcal{R}_{r}, \rho_{1}\right)$ and $\left(\mathcal{R}_{r}, \rho_{2}\right)$ are Nielsen adjacent if there is a marked rose-theta graph $\left(\Gamma_{r}, \rho\right)$ such that $\rho_{i} \simeq c_{i} \circ \rho$ for $i=1,2$ for some collapses $c_{i}: \Gamma_{r} \rightarrow \mathcal{R}_{r}$. Given any two marked roses, $\left(\mathcal{R}_{r}, \rho\right)$ and $\left(\mathcal{R}_{r}, \rho^{\prime}\right)$, it is well known that there is a sequence of markings $\rho=\rho_{1}, \ldots, \rho_{n}=\rho^{\prime}$ such that $\left(\mathcal{R}_{r}, \rho_{i-1}\right)$ and $\left(\mathcal{R}_{r}, \rho_{i}\right)$ are Nielsen adjacent for $i=2, \ldots, n$. For instance, see [14].

We will prove the theorem by showing that if $\left(\mathcal{R}_{r}, \rho_{1}\right)$ and $\left(\mathcal{R}_{r}, \rho_{2}\right)$ are Nielsen adjacent, then $x_{\rho_{1}}=x_{\rho_{2}}$. Notice that the collection $\left\{x_{\mathrm{id} \cdot \phi}\right\}$ is invariant under the $\operatorname{action} \operatorname{by} \operatorname{Out}\left(\mathbb{F}_{r}\right)$. Hence this also shows that the action of $\operatorname{Out}\left(\mathbb{F}_{r}\right)$ on $\widehat{x}^{1}\left(\mathbb{F}_{r}\right)$ has a global fixed point.

To this end, let $\left(\Gamma_{r}, \rho\right)$ be the marked rose-theta graph such that $\rho_{i} \simeq c_{i} \circ \rho$. We need to introduce a separating edge to take advantage of the shortcuts utilized in $\S 10$. Let $\widehat{\Gamma}_{r}$ be the graph obtained from blowing up the vertex $v$ in $\Gamma_{r}$. Specifically, in $\widehat{\Gamma}_{r}$ there are three vertices $v_{1}, v_{2}$ and $w$, and edges $e_{0}, e_{1}^{1}, \ldots, e_{r-2}^{1}$ and $e_{0}^{2}, e_{1}^{2}, e_{3}^{2}$. The edges are attached via the following table.

\begin{tabular}{c|c|c} 
& $o$ & $\tau$ \\
\hline$e_{0}$ & $v_{1}$ & $v_{2}$ \\
\hline$e_{i}^{1}$ & $v_{1}$ & $v_{1}$ \\
\hline$e_{i}^{2}$ & $v_{2}$ & $w$ \\
\hline
\end{tabular}

See Figure 8. We adopt the notation from $\S 10$ for $\widehat{\Gamma}_{r}$.

Let $c: \widehat{\Gamma}_{r} \rightarrow \Gamma_{r}$ be the map that collapses the edge $e_{0}$. There is a marking $\hat{\rho}: \mathcal{R}_{r} \rightarrow$ $\widehat{\Gamma}_{r}$ such that $c \circ \hat{\rho} \simeq \rho$. Viewing $\bar{X}^{1}\left(\widehat{\Gamma}_{r}, \hat{\rho}\right)$ as a subset of $[0, \infty]^{r+2}$, there are two embeddings corresponding to $\rho_{1}$ and $\rho_{2}$ denoted $\varepsilon_{1}, \varepsilon_{2}: \widehat{\mathcal{M}}^{1}\left(\mathcal{R}_{r}\right) \rightarrow[0, \infty]^{r+2}$ where $\varepsilon_{i}(\ell)^{0}=\varepsilon_{i}(\ell)\left(e_{i}^{2}\right)=0$ for $i=1,2$.

Let $S \subset[r]$ denote the set of edges $\left\{c_{i}\left(e_{1}^{1}\right), \ldots, c_{i}\left(e_{r-2}^{1}\right)\right\}$ in $\mathcal{R}_{r}$. Notice that this set is independent of $i$. Both $\varepsilon_{1}\left(\mathcal{M}_{S}^{1}\right)$ and $\varepsilon_{2}\left(\mathcal{M}_{S}^{1}\right)$ are faces of $\mathcal{M}_{1,2}^{1} \subset \widehat{\mathcal{M}}^{1}\left(\widehat{\Gamma}_{r}\right)$. Hence by Proposition 10.6(2), we have that $x_{\rho_{1}}=x_{\rho_{2}}$.

Acknowledgements. The authors thank the Institute for Computational and Experimental Research in Mathematics (ICERM) for hosting the workshop Effective and Algorithmic Methods in Hyperbolic Geometry and Free Groups, at which work on this project began. We also thank Jing Tao for suggesting Question 1.8 and the referee for a careful reading and for helpful comments. The first author is supported by NSF grant DMS-1807319. The second author is supported by Simons Foundation Grant No. 316383. The third author is supported by Simons Foundation Grant No. 637880.

\section{REFERENCES}

[1] Y. Algom-Kfir. Strongly contracting geodesics in outer space. Geom. Topol. 15(4) (2011), 2181-2233.

[2] Y. Algom-Kfir and M. Bestvina. Asymmetry of outer space. Geom. Dedicata 156 (2012), 81-92. 
[3] Y. Algom-Kfir, E. Hironaka and K. Rafi. Digraphs and cycle polynomials for free-by-cyclic groups. Geom. Topol. 19(2) (2015), 1111-1154.

[4] L. Bers. An extremal problem for quasiconformal mappings and a theorem by Thurston. Acta Math. 141(1-2) (1978), 73-98.

[5] M. Bestvina. The topology of Out $\left(F_{n}\right)$. Proc. Int. Congr. of Mathematicians, Vol. II (Beijing, 2002). Ed. T. Li. Higher Education Press, Beijing, 2002, pp. 373-384.

[6] M. Bestvina. A Bers-like proof of the existence of train tracks for free group automorphisms. Fund. Math. 214(1) (2011), 1-12.

[7] M. Bestvina and M. Feighn. Outer limits. Preprint, 1992, https://www.math.utah.edu/ bestvina/eprints/ bestvina.feighn..outer_limits.pdf.

[8] M. Bestvina and M. Feighn. Hyperbolicity of the complex of free factors. Adv. Math. 256 (2014), 104-155.

[9] R. Bowen. Equilibrium States and the Ergodic Theory of Anosov Diffeomorphisms (Lecture Notes in Mathematics, 470), revised edition. Springer-Verlag, Berlin, 2008.

[10] M. Bridgeman. Hausdorff dimension and the Weil-Petersson extension to quasifuchsian space. Geom. Topol. 14(2) (2010), 799-831.

[11] M. Bridgeman, R. Canary, F. Labourie and A. Samborino. The pressure metric for Anosov representations. Geom. Funct. Anal. 25(4) (2015), 1089-1179.

[12] M. M. Cohen and M. Lustig. Very small group actions on R-trees and Dehn twist automorphisms. Topology 34(3) (1995), 575-617.

[13] M. Culler and J. W. Morgan. Group actions on R-trees. Proc. Lond. Math. Soc. (3) 55(3) (1987), 571-604.

[14] M. Culler and K. Vogtmann. Moduli of graphs and automorphisms of free groups. Invent. Math. 84(1) (1986), 91-119.

[15] D. Cvetković and P. Rowlinson. The largest eigenvalue of a graph: a survey. Linear Multilinear Algebra 28(1-2) (1990), 3-33.

[16] G. Daskalopoulos and R. Wentworth. Classification of Weil-Petersson isometries. Amer. J. Math. 125(4) (2003), 941-975.

[17] M. Dehn. Papers on Group Theory and Topology. Springer-Verlag, New York, 1987, translated from the German and with introductions and an appendix by J. Stillwell, with an appendix by O. Schreier.

[18] S. Dowdall and S. J. Taylor. Hyperbolic extensions of free groups. Geom. Topol. 22(1) (2018), 517-570.

[19] B. Farb and L. Mosher. Convex cocompact subgroups of mapping class groups. Geom. Topol. 6 (2002), 91-152.

[20] S. Francaviglia and A. Martino. Metric properties of outer space. Publ. Mat. 55(2) (2011), 433-473.

[21] V. Guirardel and G. Levitt. Deformation spaces of trees. Groups Geom. Dyn. 1(2) (2007), 135-181.

[22] L.-Y. Kao. Pressure type metrics on spaces of metric graphs. Geom. Dedicata 187 (2017), 151-177.

[23] I. Kapovich and T. Nagnibeda. The Patterson-Sullivan embedding and minimal volume entropy for outer space. Geom. Funct. Anal. 17(4) (2007), 1201-1236.

[24] I. Kapovich and I. Rivin. On the absence of McShane-type identities for the outer space. J. Algebra 320(10) (2008), 3659-3670.

[25] H. Masur and M. Wolf. The Weil-Petersson isometry group. Geom. Dedicata 93 (2002), 177-190.

[26] H. A. Masur and Y. N. Minsky. Geometry of the complex of curves. I. Hyperbolicity. Invent. Math. 138(1) (1999), 103-149.

[27] C. T. McMullen. Thermodynamics, dimension and the Weil-Petersson metric. Invent. Math. 173(2) (2008), 365-425.

[28] C. T. McMullen. Entropy and the clique polynomial. J. Topol. 8(1) (2015), 184-212.

[29] W. Parry and M. Pollicott. Zeta functions and the periodic orbit structure of hyperbolic dynamics. Astérisque 187-188 (1990), 268.

[30] F. Paulin. The Gromov topology on R-trees. Topology Appl. 32(3) (1989), 197-221.

[31] M. Pollicott and R. Sharp. A Weil-Petersson type metric on spaces of metric graphs. Geom. Dedicata 172 (2014), 229-244.

[32] D. Ruelle. Thermodynamic Formalism: The Mathematical Structures of Classical Equilibrium Statistical Mechanics (Encyclopedia of Mathematics and Its Applications, 5). Addison-Wesley, Reading, MA, 1978, with a foreword by G. Gallavotti and G.-C. Rota.

[33] J.-P. Serre. Trees (Springer Monographs in Mathematics). Springer-Verlag, Berlin, 2003, translated from the French original by J. Stillwell, corrected 2nd printing of the 1980 English translation.

[34] W. P. Thurston. Minimal stretch maps between hyperbolic surfaces. Preprint, 1998, arXiv:math/9801039.

[35] K. Vogtmann. Automorphisms of free groups and outer space. Geom. Dedicata 94 (2002), 1-31.

[36] S. Wolpert. Noncompleteness of the Weil-Petersson metric for Teichmüller space. Pacific J. Math. 61(2) (1975), 573-577. 
[37] S. Wolpert. Thurston's Riemannian metric for Teichmüller space. J. Differential Geom. 23(2) (1986), 143-174

[38] S. A. Wolpert. The Weil-Petersson metric geometry. Handbook of Teichmüller Theory, Volume II (EMS IRMA Lectures in Mathematics and Theoretical Physics, 13). Ed. A. Papadopoulos. European Mathematical Society, Zürich, 2009, pp. 47-64.

[39] B. Xu. Incompleteness of the pressure metric on the Teichmüller space of a bordered surface. Ergod. Th. \& Dynam. Sys. 39(6) (2019), 1710-1728. 